Time Domain Modelling of Electromagnetic Metasurfaces using Generalized Sheet Transition Conditions

by

Scott A. Stewart

A thesis submitted to the Faculty of Graduate and Postdoctoral Affairs in partial fulfillment of the requirements for the degree of

Master of Applied Science

in

Electrical and Computer Engineering

Carleton University

Ottawa, Ontario

(c) 2017

Scott A. Stewart 
$\underline{\text { Carleton University }}$

\section{TIME DOMAIN MODELLING OF ELECTROMAGNETIC METASURFACES USING GENERALIZED SHEET TRANSITION CONDITIONS}

presented by: STEWART Scott A.

to obtain the degree of : Masters of Applied Science

Evaluated by the thesis committee consisting of:

M. ALBERT Jacques, Ph. D., Chair

M. SMY Tom J., Ph. D., Research Supervisor

M. GUPTA Shulabh, Ph. D., Research Supervisor

M. SCHRIEMER Henry, Ph. D., External Member

M. MCGARRY Steve, Ph. D., Internal Member 


\begin{abstract}
An explicit time-domain finite-difference technique to model zero-thickness space-time modulated Huygens' metasurfaces based on the Generalized Sheet Transition Conditions (GSTCs) is proposed and numerically demonstrated. The equivalent Lorentzian electrical and magnetic surface susceptibilities of a typical all-dielectric Huygens' unit cell, $\chi_{\mathrm{ee}}$ and $\chi_{\mathrm{mm}}$ respectively, are mapped for a range of material permittivites, which is modulated in both space and time. The problem is formulated using a set of second-order differential equations in time with non-constant coefficients. The field solutions are then solved using an explicit finitedifference time-domain technique and propagated both in the time domain and the frequency domain. Several examples of a metasurface with static or space-time modulation are shown for normally incident plane and Gaussian beams, showing the scattered field solutions. While the time-modulated metasurface generated new colinearly propagating temporal harmonics, the introduction of a space-time modulation caused the generated harmonics to travel with differing angles of refraction.
\end{abstract}




\section{TABLE OF CONTENTS}

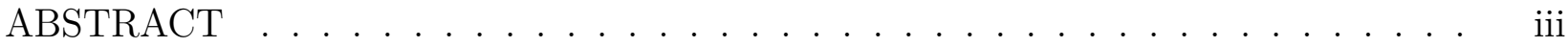

TABLE OF CONTENTS ...................... . . iv

LIST OF FIGURES . . . . . . . . . . . . . . . . . . . . . . . vi

Chapter 1 Motivation \& Background . . . . . . . . . . . . . . . . . . . 1

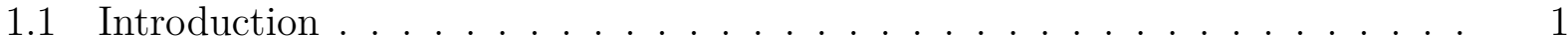

1.2 General Sheet Transition Conditions (GSTCs) . . . . . . . . . . . . 2

1.3 Objectives \& Approach . . . . . . . . . . . . . . . . . . . 4

1.4 Thesis Contributions . . . . . . . . . . . . . . . . 7

1.5 List of Publications . . . . . . . . . . . . . . . . . . . 9

Chapter 2 Background Material . . . . . . . . . . . . . . . . . . . . 10

2.1 Metasurface Modelling . . . . . . . . . . . . . . . . . . . . . . . 10

2.1.1 Huygens' Metasurfaces . . . . . . . . . . . . . . . . . . . 10

2.1.2 Finite Difference Formulation _ . . . . . . . . . . . . . . . 15

2.1.3 Extension for Time Varying Metasurface Parameters . . . . . . . . . 19

2.2 Propagation Modelling . . . . . . . . . . . . . . . . . . . 20

2.2 .1 Surface as Boundary . . . . . . . . . . . . . . . 20

2.2.2 Fourier Propagation for Single Frequency . . . . . . . . . . . . . . 21

2.2 .3 2D Yee Cell formulation . . . . . . . . . . . . . . . . . 22

Chapter 3 Method and Formulation . . . . . . . . . . . . . . . 25

3.1 Susceptibility Extraction . . . . . . . . . . . . . . . . . 25

3.2 Field Equations and Finite-Difference Formulation . . . . . . . . . . . . . 28

3.2.1 Analytical Formulation of Space-Time Modulated Metasurfaces . . . 28

3.2 .2 Finite Difference Formulation . . . . . . . . . . . . . . . . . 30

3.3 Internal Boundary Yee-cell Model . . . . . . . . . . . . . . . . . . . . . . . . 34

3.4 Space-Time Modulated Metasurface . . . . . . . . . . . . . . . . . . . . 37

3.5 Spatial and Temporal Fourier Transform Analysis . . . . . . . . . . . 38

Chapter 4 Results . . . . . . . . . . . . . . . . . . . 40

4.1 Single Point Simulations . . . . . . . . . . . . . . . . . . . 40

4.1 .1 Static Surface . . . . . . . . . . . . . . . . . . . 40 
4.1.2 Time-Varying Surface . . . . . . . . . . . . . . . . 41

4.2 2D Simulations . . . . . . . . . . . . . . . . . . . 42

4.2.1 Fourier Propagation Examples . . . . . . . . . . . . . . 43

4.2 .2 Yee Cell . . . . . . . . . . . . . . . . . . . 46

Chapter 5 Conclusions and Future Work . . . . . . . . . . . . . . . . 54

5.1 Discussion . . . . . . . . . . . . . . . . . . . . 54

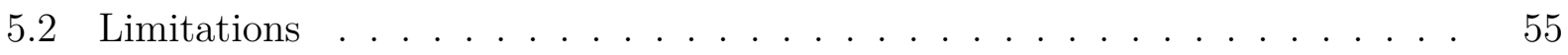

5.3 Future Work . . . . . . . . . . . . . . . . . 56

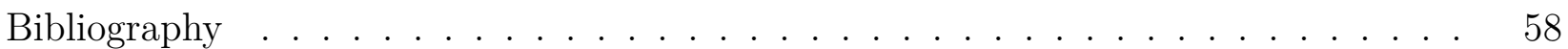

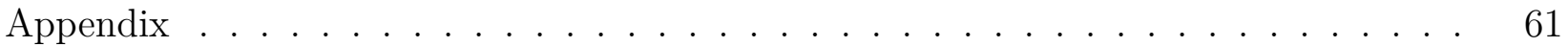




\section{LIST OF FIGURES}

Figure 2.1 A typical configuration of a zero-thickness Huygen's metasurface, consisting of orthogonal electric (p) and magnetic (m) dipole moments, excited with a normally incident plane-wave resulting in reflected and transmitted fields governed by (1.2). Figure sourced from [1]. . . . . .

Figure 2.2 Huygens' source metasurface based on all-dielectric resonators and its constitutive susceptibility parameters. a) A unit cell model with periodicity in both the $x$ - and $y$ - directions. b) Typical amplitude transmission and reflection response, computed using FEM-HFSS, when the two dipole moments $\mathbf{p}$ and $\mathbf{m}$ are frequency aligned. c) Extracted electric susceptibility function (magnetic susceptibility not shown for simplicity) from (b) using (2.1) and the Lorentz curve-fit using (2.2). Design parameters: $r_{1}=300 \mathrm{~nm}, t=220 \mathrm{~nm}, \Lambda=666 \mathrm{~nm}, n_{2}=1.66$ (Silica) and $n_{1}=3.45$ and $\tan \delta=0.001$ (Silicon). Figure sourced from $[1] \ldots \ldots \ldots \ldots \ldots \ldots \ldots \ldots$

Figure 2.3 A coupled RLC resonator circuit model as an equivalent circuit model representation of the Huygens' metasurface of Fig. 2.2. Figure sourced from [1]. . . . . . . . . . . . . . . . . . . . . . . . . . .

Figure 2.4 Simulation setup for the finite-difference technique showing the numerical domain and the surrounding boundary conditions. Shaded regions on the left and right represent the $2 \mathrm{D}$ discretized region where Maxwell's equations are solved on a conventional Yee cell. Figure

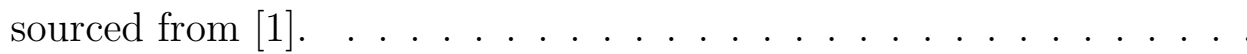

Figure 2.5 Yee cell lattice in $2 \mathrm{D}$ with $E_{y}, H_{x}$ and $H_{z}$ fields. $i$ is the index in space along the $z$ direction, $j$ is the index in space along the $x$ direction, and $\Delta$ is the spatial distance between two field nodes. . . . . . . . . . . . 24

Figure $3.1 \quad$ All-dielectric unit cell for different material permittivities, $\epsilon_{r}=(11,11.5,12.2,13)$.

a) Two different unit cell geometries showing the transverse view with wave propagation along $z$. b) Transmission and reflection responses for varying permittivity values, and c) the corresponding extracted complex electric and magnetic susceptibilities, $\tilde{\chi}_{\mathrm{ee}}$ and $\tilde{\chi}_{\mathrm{mm}}$, respectively. The full-wave simulation parameters are: unit cell $\# 1-d_{2}=80 \mathrm{~nm}$, unit cell \#2 - $d_{2}=60 \mathrm{~nm} . d_{1}=560 \mathrm{~nm}$, substrate thickness $h=$ $220 \mathrm{~nm}$ and $\epsilon_{h}=2.76$ for both cases. Figure sourced from [2]. . . . . 
Figure 3.2 The variation of the electric and magnetic resonant frequencies of the three metasurface unit cells of Fig. 3.1, as a function of material permittivity $\epsilon_{r}$, for both the first and second Lorentzian contributions. The center frequency and the bandwidth of the incident signal are $f_{s}=230 \mathrm{THz}$ and $\Delta f \approx 50 \mathrm{THz}$, respectively. Figure sourced from [2].

Figure 3.3 Simulation setup showing the numerical domain for a time-domain simulation including a finite-size metasurface of length $\ell$, and the surrounding boundary conditions. Shaded regions on the left and right represent the 2D discretized region where Maxwell's equations are solved on a conventional Yee cell. Figure sourced from $[2] \ldots \ldots \ldots$

Figure 3.4 Simulation flow chart illustrating the key steps in solving a space-time modulated metasurface using the proposed method. Figure sourced from $[2] \ldots \ldots \ldots \ldots \ldots$

Figure 4.1 Response of a linear time-invariant metasurface, showing the instantaneous E-field of the time-domain reflected and transmitted fields at the metasurface. . . . . . . . . . . . . . . .

Figure 4.2 Response of a time modulated metasurface with $\omega_{m}=0.025 \omega_{0}=$ $2 \pi(5.75 \mathrm{THz})$ and $\Delta_{p}=0.1$, showing the instantaneous E-field of the time-domain reflected and transmitted fields at the metasurface. . . .

Figure 4.3 Temporal Fourier transform of the output fields from the time modulated metasurface with $\omega_{m}=0.025 \omega_{0}=2 \pi(5.75 \mathrm{THz})$ and $\Delta_{p}=0.1$ once it has reached steady state. This shows (a) the power of the transmitted field as a function of frequency and (b) the power of the reflected field as a function of frequency, both expressed in $\mathrm{dB}$. . . . .

Figure 4.4 Fourier propagated fields in the transmission region for the static metasurface. The maximum field is $\max \left\{\left|E_{t}(x, z, \omega)\right|\right\}=0.8615 \ldots \ldots$.

Figure 4.5 Output field characteristics of a matched space-time modulated metasurface excited with an normally incident Gaussian beam. (a) Spatial Fourier transform at the output of the metasurface at $z=0_{+}$ for first few harmonics, normalized to the max value of the largest generated harmonic. (b) The refraction angle of each harmonic using (3.26). Metasurface parameters: $25 \mu \mathrm{m}$ in size, and mesh size $\Delta x$ of $125 \mathrm{~nm}$. The Gaussian beam parameters: $\omega_{0}=2 \pi(230 \mathrm{THz})$, and standard variation of $\sigma_{x}=5 \mu \mathrm{m}$. Modulation parameters: $\Delta_{m}=0.1$, $\beta_{m}=4 \pi \times 10^{5} \mathrm{rad} / \mathrm{m}$ and $\omega_{m}=0.025 \omega=2 \pi(5.75 \mathrm{THz})$. Figure sourced from $[3] . \ldots \ldots \ldots \ldots \ldots \ldots \ldots \ldots$ 
Figure 4.6 Fourier propagated fields in the transmission region of the metasurface of Fig. 4.5 showing the first up- and down-converted harmonics. $\max \left\{\left|E_{t}\left(x, z, \omega_{+1}\right)\right|\right\}=0.5942$, and $\max \left\{\left|E_{t}\left(x, z, \omega_{-1}\right)\right|\right\}=0.6403 \quad$.

Figure 4.7 Response of a linear time-invariant uniform metasurface, showing the instantaneous E-field and H-field distributions, corresponding timedomain reflected and transmitted fields at the field-probing locations $\left(x_{0}, \pm z_{0}\right)$, and the spatial Fourier transform of the fields in a transverse cross-section at $z=z_{0}$, for the two cases of (a) normally incident plane-wave, and (b) normally incident Gaussian-beam. The simulation parameters are as follows: $\mathrm{CW}$ frequency $f_{s}=230 \mathrm{THz}, \ell=25 \mu \mathrm{m}$, Gaussian field at the input $E_{y}\left(x, z=0_{-}\right)=\exp \left[-\left(x-x^{\prime}\right)^{2} / \sigma_{x}^{2}\right]$, with $x^{\prime}=25 \mu \mathrm{m}, \sigma_{x}=5 \mu \mathrm{m}$. The rising Gaussian edge for plane-wave case is $\sigma_{t}=100 \mathrm{fs}$. Meshing $n_{x}=400$. The metasurface parameters for are extracted from the unit cell \#2 of Fig. 3.1. Figure sourced from [2]. .

Figure 4.8 Transmitted and reflection fields from a linear time-invariant metasurface for the case of a pulsed (broadband) Gaussian beam. (a) Spatial E-fields in the entire simulation region at two time instants $t_{1}=0.2 \mathrm{ps}$, and $t_{2}=0.4$ ps. (b) Time-domain incident, reflected and transmitted fields at the probe locations in the transmission and reflection regions $\left[\left(x_{0},-z_{0}\right)\right.$ and $\left.\left(x_{0},+z_{0}\right)\right]$, respectively. (c) The convergence plot of the simulation, where the average energy in the transmission and reflection regions converges at each time instant, for increasing value of the mesh density (related to $n_{x}$, where $n_{z}=3 n_{x}$ ). The gaussian pulse width parameter is $\sigma_{t}=50 \mathrm{fs}$. Figure sourced from [2]. . . . . . . . . .

Figure 4.9 Response of a time-only modulated (uniform) metasurface for a normally incident Gaussian pulsed Gaussian beam with pumping frequency $f_{m}=0.025 f_{0}=5.75 \mathrm{THz}$ and $\Delta_{p}=0.1$, showing a) the time-domain E-fields at $\left(x_{0}, \pm z_{0}\right)$ and its spatial distribution in the transmission and reflection regions at a given time instant, (b) transmission and reflection temporal spectrum at $\left(x_{0}, \pm z_{0}\right)$, and (c) E-field distribution in the transverse plane at $z= \pm z_{0}$, corresponding to transmission and reflection fields, respectively. Only first few spectral components are shown, for clarity. $\mathcal{F}_{t}\{\cdot\}$ and $\mathcal{F}_{x}\{\cdot\}$ denote temporal and spatial Fourier transforms, respectively. The gaussian pulse width parameter is $\sigma_{t}=600 \mathrm{fs}$.

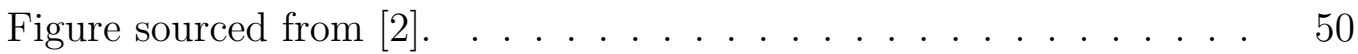


Figure 4.10 Response of a space-time modulated metasurface with space-time varying permittivity according to (3.1), showing (a) the scattered fields at two time instants. (b) Transmitted and reflected fields at $z= \pm z_{0}$ for individual harmonics, (c) the spatial Fourier transform of the transmitted and reflected fields at $z=0_{+}$and $z=0_{-}$, respectively. (d) The refraction angles of each each harmonic components in reflection and transmission. The pumping frequency $\omega_{m}=0.025 \omega_{0}=5.75 \mathrm{THz}$ and spatial modulation $\beta_{m}=10 \pi / \ell . \mathcal{F}_{t}\{\cdot\}$ and $\mathcal{F}_{x}\{\cdot\}$ denote temporal and spatial Fourier transforms, respectively. Figure sourced from [2]. . . 


\section{Chapter 1 Motivation \& Background}

\section{$1.1 \quad$ Introduction}

Metasurfaces are two dimensional arrays of sub-wavelength electromagnetic scatterers [4][5]. Similar to the more general volumetric metamaterial structures, the electromagnetic properties of the scatterers can be engineered in order to control both the spatial wavefronts of incident waves as well as their temporal shapes. The metasurface can impart a mixture of amplitude and phase transformations, which transforms an incident field into the desired transmitted and reflected fields [6][7][8]. This nature allows them to be useful in a diverse range of applications, such as in lensing, imaging [9][5], field transformations [10], cloaking [11] and holograming [12].

One important classification of metasurface is the Huygens' metasurface, which has recently been a focus of study for their impedance matching capabilities with free-space. Huygens' metasurfaces, which are the focus of this thesis, are constructed out of electrically small Huygens' sources. The structure of these sources allows optimal interactions between the electric and magnetic dipolar moments, which cancels backscattering [13]. An example of an efficient implementation of Huygens' metasurfaces is based on all-dielectric resonators $[14][15]$.

A metasurface can also be designed to be tunable in order to change some of its constitutive parameters, such as the susceptibility densities. A simple example of this is a generalized refracting metasurface, where the properties of the sub-wavelength scatterers are varied as a periodic function of space over the metasurface aperture [16]. Due to the spatial gradient of these parameters this surface can be described as a space modulated metasurface. An extension of this is when the constitutive parameters are varied not only as a function of space but are periodically varied in both space and time, where the temporal modulation occurs from actively tuning the metasurface. These space-time modulated metasurfaces have been a growing area of interest due to their ability to generate frequency conversions due to the complex wave interactions [17][18].

The computational modelling of both the frequency generation as well as the amplification that is caused by these complex wave interactions provides an interesting field of work as well as a numerical challenge. A metasurface with a sub-wavelength thickness can be rigorously modelled as an ideal zero-thickness surface utilizing the general sheet transition conditions (GSTCs) [19]. The surface can be converted from a multiple interface problem 
into a single interface problem by using an explicit incident field in the time domain. The shift to the time domain will also allow the proper modelling of space-time modulation across the metasurface for a given excitation.

Previous works have attempted to tackle the simulation of metasurfaces using a variety of techniques. One method to model the interactions of a metasurface is to simulate the surface in the frequency-domain, which captures the effects of the surface at steady state for a single frequency [20]. This model is able to properly simulate the propagation of a static metasurface, but it is not able to handle the complex interactions that occur when the metasurface is modulated in both space and, more importantly, time. These calculations cannot be performed exclusively in the frequency domain due to the harmonic generation that is expected from a temporally modulated surface.

A more recent example is a paper that discusses a rudimentary implementation of the metasurface in the time-domain using finite-difference techniques [21]. The model discussed in [21] uses frequency-independent susceptibilities that do not properly capture the behaviour of a practical metasurface. Although this can be rectified to follow more closely with the behaviour of the metasurface, the formulation itself was found to be in considerable need of improvement.

\subsection{General Sheet Transition Conditions (GSTCs)}

Metasurfaces produce a spatial discontinuity in both the magnitude as well as the phase of a wave. Because the metasurface has a sub-wavelength thickness it can be modelled as a zero-thickness surface using the GSTCs, which were developed by Idemen in [19] and were later applied to metasurface problems in [22]. For a Huygens' surface that is lying in the $x-y$ plane, the four GSTC equations can be written in the time-domain as,

$$
\begin{gathered}
\hat{\mathbf{z}} \times \Delta \mathbf{H}=\frac{d \mathbf{P}_{\|}}{d t}-\hat{\mathbf{z}} \times \nabla_{\|} M_{z} \\
\Delta \mathbf{E} \times \hat{\mathbf{z}}=\mu_{0} \frac{d \mathbf{M}_{\|}}{d t}-\nabla_{\|}\left(\frac{P_{z}}{\epsilon_{0}}\right) \times \hat{\mathbf{z}}, \\
\hat{\mathbf{z}} \cdot \Delta D=-\nabla \cdot P_{\|} \\
\hat{\mathbf{z}} \cdot \Delta B=-\mu_{0} \nabla \cdot M_{\|}
\end{gathered}
$$

where $\Delta \psi$ is the difference between the fields on the two sides of the metasurface for all of

the vector components of the field, $\nabla_{\|}=\frac{\partial}{\partial x} \hat{\mathbf{x}}+\frac{\partial}{\partial y} \hat{\mathbf{y}}$, and $P_{z}$ and $M_{z}$ are the polarizations of the field normal to the surface. $P_{\|}$and $M_{\|}$are the electric and magnetic surface polariza- 
tion densities in the plane of the metasurface, and depend on the total average field across the metasurface. The first two equations alone are able to properly model the metasurface because longitudinal fields can be determined from the transverse fields uniquely [23], therefore the other two equations are not used in the model formulation. Likewise, because the metasurface is assumed to be restricted to tangential polarizations only, the first two GSTC equations can be simplified to

$$
\begin{gathered}
\hat{\mathbf{z}} \times \Delta \mathbf{H}(x, t)=\frac{d \mathbf{P}_{\|}(x, t)}{d t} \\
\Delta \mathbf{E}(x, t) \times \hat{\mathbf{z}}=\mu_{0} \frac{d \mathbf{M}_{\|}(x, t)}{d t},
\end{gathered}
$$

In its most general form, each of the polarization densities induced on the metasurface may be expressed as a function of both the average electric and magnetic fields $\mathbf{E}_{\text {avg }}$ and $\mathbf{H}_{\mathrm{avg}}$, respectively, through the surface susceptibilities. The polarization densities can be expressed in free-space as

$$
\begin{gathered}
\overrightarrow{\mathbf{P}}_{\|}=\epsilon_{0} \overline{\bar{\chi}}_{\mathrm{ee}} \vec{E}_{a v g}+\sqrt{\epsilon_{0} \mu_{0}} \overline{\bar{\chi}}_{\mathrm{em}} \vec{H}_{a v g} \\
\overrightarrow{\mathbf{M}}_{\|}=\overline{\bar{\chi}}_{\mathrm{mm}} \vec{H}_{\text {avg }}+\sqrt{\frac{\epsilon_{0}}{\mu_{0}}} \overline{\bar{\chi}}_{\mathrm{me}} \vec{E}_{\text {avg }}
\end{gathered}
$$

where $\overline{\bar{\chi}}_{\mathrm{ee}}, \overline{\bar{\chi}}_{\mathrm{mm}}, \overline{\bar{\chi}}_{\mathrm{em}}$, and $\overline{\bar{\chi}}_{\text {me }}$ are the surface susceptibilities of the metasurface. These susceptibilities are generally tensors, meaning that a field with a polarization in the $x$ direction can excite the polarization density in the $y$ direction and vice-versa, which may cause a rotation of the polarization. For simplification, it is assumed that the susceptibilities of the metasurface are scalar values, such that an excitation in any given polarization will not generate a rotation. It is also assumed for convenience that the susceptibilities are independent of the angle of incidence. The extension to full tensors is however straightforward.

Another simplification that can be made is to assume that the bi-anisotropic terms are zero, i.e. $\chi_{\mathrm{em}}=\chi_{\mathrm{me}}=0$. This condition coupled together with the scalar susceptibilities assumes that the metasurface that is being analyzed is isotropic in nature.

With these simplifications it is easier to identify the conditions that can lead to different behaviours that may be a point of interest. The most readily apparent example is the condition of losslessness for a metasurface. The general formulation for a lossless material is rather complex, factoring in all of the susceptibility tensors [23]. Because the metasurface is assumed to be isotropic, the equations to determine losslessness are greatly simplified. 
The surface susceptibilities are analogous to volumetric susceptibilities, used to calculate the surface polarization densities instead of the polarization fields. In a traditional bulk material, the permittivity ${ }^{1}$ can be written as a complex number in the form of $\epsilon=\epsilon^{\prime}+i \epsilon^{\prime \prime}$, where $\epsilon^{\prime}$ and $\epsilon^{\prime \prime}$ are the real and imaginary components of the permittivity, respectively. $\epsilon^{\prime \prime}$ controls the gain and the loss that a given field experiences when propagating through the material. Assuming that there is a $e^{i \omega t}$ time convention, a positive value increases the field when traveling through the region and a negative value presents a decrease, or loss, of the field. Because $\epsilon^{\prime \prime}$ controls the gain or the loss of the material, setting the value to zero would cause the material in question to be lossless. Therefore a purely real value of $\epsilon$ gives a lossless bulk material. The same argument can be made for the permeability of the material, $\mu$.

Because of this relationship between the surface susceptibilities and the bulk susceptibilities, it can be reasonably assured that an isotropic metasurface is considered lossless if its scalar surface susceptibilities are purely real, i.e. $\chi_{\mathrm{ee}}=\operatorname{Re}\left\{\chi_{\mathrm{ee}}\right\}$ and $\chi_{\mathrm{mm}}=\operatorname{Re}\left\{\chi_{\mathrm{mm}}\right\}$. Although this relation does not necessarily mean that the transmitted field through the metasurface will have the same power as the incident field, it does mean that the sum of the power of the transmitted and reflected fields will be the same as the incident field, for a static metasurface.

\subsection{Objectives \& Approach}

The objective of this thesis is to demonstrate the implementation and determine the scattered fields from a space-time modulated metasurface in the time-domain utilizing the GSTC equations. This presents obstacles that must be addressed when tackling this problem.

The metasurface formulation must be inherently causal, meaning that any form of outputted field has to be directly caused by a given input field. It should not be possible to have a metasurface react to an input source before the source actually interacts with the metasurface. Although a frequency-domain simulation may assume that an input and given output from the metasurface has always been at steady-state, the time domain simulation cannot anticipate what the field is going to be.

This leads into the second restriction to the model, which is that it must be able to handle any arbitrary time-domain excitation. Whereas frequency-domain simulations assume that there is only one frequency of excitation, the time-domain model should be able to handle any broad-band signal that is incident on the metasurface. This includes excitations that exhibit sudden phase shifts as well as incident fields that shift from one frequency to another.

\footnotetext{
${ }^{1}$ The permittivity is directly related to the electrical volumetric susceptibility through $\epsilon=\epsilon_{0}\left(1+\chi_{\mathrm{ee}}\right)$.
} 
The arbitrary nature of the incident field results in an output field from the metasurface that will be similarly complex. Because of this, it is advantageous to be able to observe the diffraction effects from the fields as they propagate away from the metasurface. This happens naturally since the metasurface is typically operated around the resonance frequencies of the constituting scatterers. Therefore simulation regions have to be constructed either before and after the metasurface or around the surface in order to visualize the effects that the output waveforms experience. These regions can also be used to better observe any phenomenon that is produced from the metasurface interacting with the incident field and the space-time modulation.

Coupled together with this, there are a few numerical complexities that are related to the harmonic generation produced by both the arbitrary time-domain excitation as well as the space-time modulation. In a purely time-domain simulation, a Yee-cell is required to propagate a field through space. This Yee-cell needs to have a minimum number of spatial divisions in order to propagate the field without excess distortion, and this number is dependent on the frequency, where higher frequencies require a better resolution. The mesh density for a static metasurface that experiences a monochromatic wave, thus outputting a wave with a single frequency, may be too small for the higher ordered harmonics that would propagate from the same metasurface with a space-time modulation. This means that a higher mesh density would need to be used for a modulated metasurface simulation compared to its static counterpart, increasing the amount of time it would take to simulate.

This simulation time is further exacerbated by the analysis portion of a given simulation. To analyze the harmonics that are generated from a time-domain simulation, a Fast Fourier Transform (FFT) has to be conducted. If the difference between the frequencies of the harmonics is relatively small, the frequency resolution of the FFT $\Delta f$ has to be small as well. The frequency resolution, however, is inversely proportional to the length of the simulation. Therefore in order to properly capture the harmonic content of the metasurface a long simulation has to be performed.

If the field is propagated in the time-domain, a finite sized simulation region has to be considered because of computational limitations. The boundary conditions of this simulation region have to be set so that there is no reflection from the boundaries back to the metasurface, which would otherwise cause improper results. The approach that is used is to add a Perfectly Matched Layer (PML) to each of the boundaries which absorbs the fields and eliminates reflection. The effectiveness of these PMLs are related to the length of the PML as well as the frequency of the field that is being absorbed. The PML is less effective when the frequency of the field is lower, since the field has fewer cycles traveling through the PML. 
This can be mitigated by increasing the size of the PML, but the drawback of this is that more computations have to be made per time step through the simulation. Finding a balance between the accuracy of the results and the speed of the model is a challenge that has to be addressed when preparing a simulation.

There are a few assumptions that are made in order to simplify the problem. First of all, the surface susceptibilities are assumed to be scalar, as explained before, meaning that no polarization rotation is considered. It is also assumed that the metasurface has an isotropic response and that there are no bi-anisotropic terms, i.e. $\chi_{\mathrm{em}}=\chi_{\mathrm{me}}=0$.

Another assumption of the model is that the input fields are normally incident with the metasurface with small diverging angles. If the incident wave is oblique or if the beam is widely diverging, such as a Gaussian beam with a high spread, the results of the model are not accurate. For these cases a non-linear solver would be required, which would increase the accuracy of the results at the cost of speed and is a focus of future work. This limitation holds for both static as well as space-time modulated metasurfaces.

When developing the metasurface model there were two different formulations that were explored: the Implicit method and the Explicit method. The Implicit method assumes that the metasurface can be described by inserting the metasurface directly into the simulation region, which in the case of the Yee cell would modify the surrounding field nodes' update equations by using the GSTC equations. This method does not assume any forward- or backward-propagating waves, but instead simply uses the electric and magnetic field nodes themselves. The advantage of this is that one could propagate a wave from a source towards the metasurface and the field nodes would naturally generate the transmitted and reflected fields.

Problems arose when attempting to implement the Implict model. Due to the definition of the GSTCs requiring a zero-thickness region, different methods tried to manipulate the placement of the field equations inside of a standard Yee cell. Solutions such as duplicating a field node on either side of the metasurface would result in spurious reflections for a surface that was supposed to have no reflection at all. Although it was attempted in different publications [21] an accurate model is still under development and will be discussed in future works.

This led to the Explicit method, which is studied and utilized in this thesis. Instead of using arbitrary fields, the Explicit method assumes that there is a known incident wave on the $-z$ side of the metasurface, and that this yields a transmitted wave in the $+z$ direction and a reflected wave in the $-z$ direction $^{2}$. The waves are applied as boundary conditions

\footnotetext{
${ }^{2}$ Assuming that the metasurface lies in the $x-y$ plane at $z=0$.
} 
either on the sides of a simulation region or inside of the region itself. These waves are then assumed to propagate independent of the incident wave and each other. One drawback is that it is not able to handle reflections in the bulk regions due to the hard-source nature of the formulation. If the bulk regions are not reflective, the strengths of this method far outweigh the current inaccuracies of the Implicit method.

In this thesis, the Explicit time-domain metasurface formulation for an arbitrary field and space-time modulation is developed. Several different ways to visualize the field response, such as a hard boundary in a typical Yee cell or the conversion into the frequency-domain for Fourier propagation, are explored in rigorous detail. A past circuit-based model of the metasurface is also discussed briefly, and the conversion to a more physical model is explained.

In order to simulate a surface with the proper response in the time-domain, the constitutive parameters from a typical metasurface cell are extracted using commercial software. These parameters are used in different simulation setups in order to rigorously show the effects of the metasurface with different types of modulation, as well as different ways to analyze the surface itself. Single-point simulations, where only a single point along a uniform metasurface is analyzed for a wave with normal incidence, are conducted for both a nonmodulated and a time modulated metasurface. This gives a good understanding of what the metasurface does without taking into account the effects of diffraction from propagation.

2D simulations are then performed for a variety of situations. First, the Fourier propagation techniques are tested by analyzing the transmitted field on a metasurface with a monochromatic incident Gaussian beam under two different types of modulation: no modulation and space-time modulation. The static and space-time modulation simulations are then repeated for a 2D Yee cell, demonstrating the fringing effects that cannot be seen through a frequency-domain simulation. Finally, the effects of the metasurface with a broadband Gaussian pulse incident field are analyzed for a static and a time-only modulated metasurface in order to prove that the surface can work for a complicated input waveform.

\subsection{Thesis Contributions}

Through working on this thesis, I have developed several tools to model metasurfaces as well as methods to analyze the computed results. One of the crucial things that I developed was the method to determine the spatial profile of select frequencies along a line in space in a broadband time-domain field. This method to visualize the field profiles for different frequencies proved to be useful when analyzing the output of a space-time modulated metasurface, especially when the different harmonics are very similar in terms of frequency. 
I then expanded the method by taking the spatial Fourier transform of the spatial profiles for the separate frequencies. This additional step proved useful in two different ways, the first of which is verification. If the metasurface outputs a plane wave that propagates through free-space, the analyzed spatial frequency distribution at a distance away from the surface should still be very close to the spatial frequency distribution of the same wave immediately after the surface, even if the spatial profile itself is not the same. This allows us to verify that the propagation methods that are used work properly.

The other use is to determine the angle of refraction that the given harmonic will propagate at. By taking the spatial frequency with the highest power, the angle of refraction can be easily calculated and then compared with the theoretical values.

The other method that I developed in this thesis is an expansion of the Fourier propagation techniques of [24]. In the normal Fourier propagation method, only fields with a single frequency could be propagated in the frequency domain. The method that I used to analyze a broadband signal was to take the spatial profile of each of the harmonic frequencies in question and to separately propagate them using the Fourier propagation techniques in different regions. Although this was a simple extension, it was useful in analyzing the broadband output fields from space-time modulated metasurfaces.

This thesis outlines the creation of the first time-domain metasurface model that takes both causality as well as dispersion into account for realistic Huygens' metasurfaces. The model evolved from the previous circuit-based model into one that better captured the physics of the metasurface. I then applied the model so that it was able to properly handle any arbitrary time-domain excitation with a normal incidence and an arbitrary space-time modulation. While there are several assumptions made to simplify the formulation, extensions to more general cases are possible with minimal effort. 


\subsection{List of Publications}

[1] S. Gupta, S. A. Stewart, and T. J. Smy, "Space-Time Modulation Induced Non-reciprocity in Electromagnetic Metasurfaces," Physical Review A, under review.

[2] S. Gupta, T. J. Smy, and S. A. Stewart, "Floquet-Mode Solutions of Space-Time Modulated Huygens' Metasurfaces," IEEE Trans. Antennas Propag., under review.

[3] S. A. Stewart, T. J. Smy, and S. Gupta, "Finite-Difference Time-Domain (FDTD) Modelling of Space-Time Modulated Metasurfaces," IEEE Trans. Antennas Propag., under review.

[4] S. A. Stewart, T. J. Smy, and S. Gupta, "Semi-analytical finite-difference technique for steady-state field characterization of space-time modulated huygens' metasurfaces," in 2017 IEEE International Symposium on Antennas and Propagation (APSURSI), July 2017.

[5] T. J. Smy, S. A. Stewart, and S. Gupta, "Time-domain circuit modelling of huygens' metasurfaces," in 2017 IEEE International Symposium on Antennas and Propagation (APSURSI), July 2017.

[6] S. A. Stewart, S. Gupta, and T. J. Smy, "Rigorous time-domain modelling of electromagnetic metasurfaces," in 2017 Photonics North, May 2017. 


\section{Chapter 2 Background Material}

The background material is split up into two separate sub-sections: Sec. 2.1 (Metasurface Modelling) and Sec. 2.2 (Propagation Modelling). The Metasurface Modelling section closely follows the background material found in [1], which is the foundation that the thesis builds upon. Here the method to simulate a Huygens' metasurface using a circuit-based model is reviewed along with the extension to model a time-varying metasurface.

Secondly, the Propagation Modelling section gives the necessary background material that is needed in order to propagate the output fields from the simulated metasurface using a variety of different techniques. These techniques include propagating a broadband timedomain field through space in both the time domain as well as the frequency domain.

\subsection{Metasurface Modelling}

\subsubsection{Huygens' Metasurfaces}

A plane wave with a frequency of $\omega_{0}$ is normally incident on a metasurface. As illustrated in Fig. 2.1, the metasurface interacts with the input field and generates both transmitted and reflected fields in general. The metasurface is typically characterized using effective surface susceptibility densities, $\chi$, which in its most general form is a full tensor [6], capturing all possible wave interactions.

A typical Huygens' all-dielectric metasurface unit cell consists of a high-dielectric elliptical resonator (index $n_{1}$ ) embedded in a host medium of index $n_{2}$, as shown in Fig. 2.2(a). A Huygens' metasurface is constructed out of a periodic lattice of unit cells along the $x$ - and $y$ - directions and is subwavelength in dimension, i.e. $\Lambda<\lambda_{0}$, to ensure only a zeroth order propagation mode. Its typical transmission and reflection amplitude response is shown in Fig. 2.2(b), computed using the commercial software "finite element method high frequency structural simulator" (FEM-HFSS). Using the computed complex transmission and reflection response of this unit cell, its corresponding surface susceptibilities can be extracted by

$$
\begin{gathered}
\tilde{\chi}_{\mathrm{ee}}\left(\omega_{0}\right)=\frac{2 j}{k_{0}} \frac{\tilde{E}_{t}+\tilde{E}_{r}-\tilde{E}_{0}}{\tilde{E}_{t}+\tilde{E}_{r}+\tilde{E}_{0}}=\frac{2 j}{k_{0}}\left(\frac{T+R-1}{T+R+1}\right) \\
\tilde{\chi}_{\mathrm{mm}}\left(\omega_{0}\right)=\frac{2 j}{k_{0}} \frac{\tilde{E}_{t}-\tilde{E}_{r}-\tilde{E}_{0}}{\tilde{E}_{t}-\tilde{E}_{r}+\tilde{E}_{0}}=\frac{2 j}{k_{0}}\left(\frac{T-R-1}{T-R+1}\right),
\end{gathered}
$$




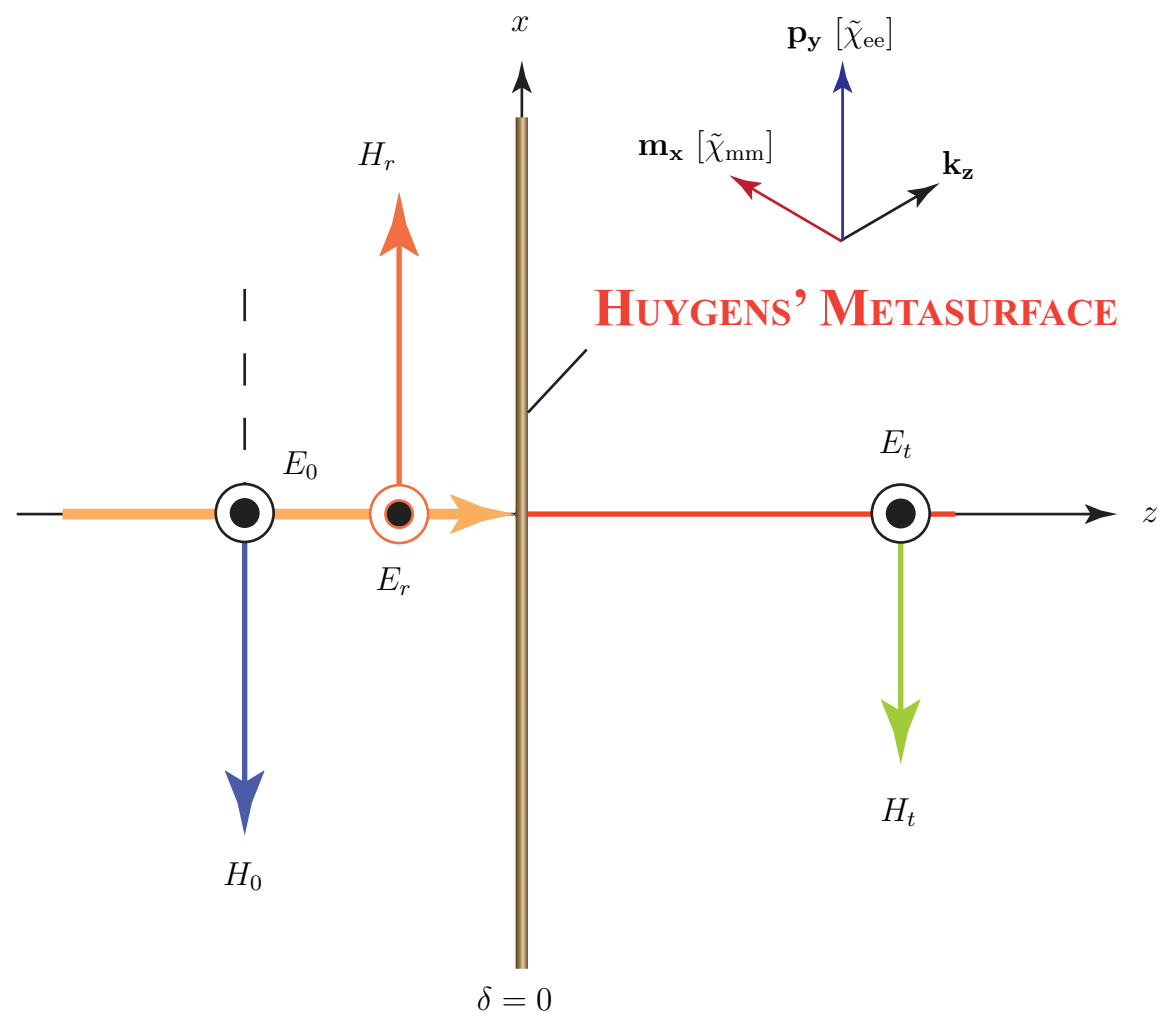

Figure 2.1 A typical configuration of a zero-thickness Huygen's metasurface, consisting of orthogonal electric $(\mathbf{p})$ and magnetic $(\mathbf{m})$ dipole moments, excited with a normally incident plane-wave resulting in reflected and transmitted fields governed by (1.2). Figure sourced from [1]. 
where $T=\tilde{E}_{t} / \tilde{E}_{0}$ and $R=\tilde{E}_{r} / \tilde{E}_{0}$ are the frequency-domain transmission and reflection transfer functions, respectively and $\tilde{\psi}$ is the Fourier transform of the temporal fields $\psi$. Fig. 2.2(c) shows the extracted surface susceptibilities, where only the electric susceptibilities are shown for simplicity. Since the reflection from the metasurface is non-zero, $\tilde{\chi}_{\mathrm{ee}} \neq \tilde{\chi}_{\mathrm{mm}}$ in general, across the entire frequency band of interest.

A dielectric resonator such as the one in Fig. 2.2(a) can be modelled using a standard physical Lorentz oscillator [25]. Following this model, it can be easily verified that the susceptibility functions $\tilde{\chi}_{\mathrm{ee}}$ (and $\tilde{\chi}_{\mathrm{mm}}$ ) of Fig. 2.2(c), can be accurately described using a double-Lorentz function given by

$$
\begin{aligned}
\tilde{\chi}_{\mathrm{ee}}(\omega) & =\sum_{i=1}^{2} \frac{\omega_{e p, i}^{2}}{\left(\omega_{e 0, i}^{2}-\omega^{2}\right)+i \alpha_{e, i} \omega} \\
\tilde{\chi}_{\mathrm{mm}}(\omega) & =\sum_{i=1}^{2} \frac{\omega_{m p, i}^{2}}{\left(\omega_{m 0, i}^{2}-\omega^{2}\right)+i \alpha_{m, i} \omega},
\end{aligned}
$$

as shown in Fig. 2.2(c) for electric susceptibility, where $\omega_{p}, \omega_{0}$ and $\alpha$ are the plasma frequency, resonant frequency and the loss-factor of the oscillator, respectively, and subscript $e$ and $m$ denote electric and magnetic quantities. For more complicated unit cell designs more than two Lorentzian contributions may be required to accurately capture the surface susceptibility.

\section{Lorentzian Material Response and Field Equations}

Having shown that the double-Lorentzian function accurately describes the susceptibility of a typical metasurface unit cell, the sub-wavelength thickness unit cell of Fig. 2.2 can now be modelled using an ideal zero-thickness Huygens' metasurface with a double-Lorentzian dispersion profile. To simplify the math, we shall first consider the surface susceptibilities with only a single Lorentz contribution.

In physical terms, the electric susceptibility, $\tilde{\chi}_{\text {ee }}$ relates the electric polarization response of the metasurface $\tilde{\mathbf{P}}(\omega)$ to the input electric fields $\tilde{\mathbf{E}}(\omega)$. Similarly, the magnetic susceptibility, $\tilde{\chi}_{\mathrm{mm}}$, relates the magnetic polarization response of the metasurface $\tilde{\mathbf{M}}(\omega)$ to the input magnetic fields $\tilde{\mathbf{H}}(\omega)$. Mathematically, this can be expressed in the frequency domain as

$$
\begin{aligned}
\tilde{\mathbf{P}}(\omega) & =\epsilon_{0} \tilde{\chi}_{\mathrm{ee}}(\omega) \tilde{E}(\omega)=\frac{\epsilon_{0} \omega_{e p}^{2}}{\left(\omega_{e 0}^{2}-\omega^{2}\right)+i \alpha_{e} \omega} \tilde{\mathbf{E}}(\omega) \\
\tilde{\mathbf{M}}(\omega) & =\tilde{\chi}_{\mathrm{mm}}(\omega) \tilde{H}(\omega)=\frac{\omega_{m p}^{2}}{\left(\omega_{m 0}^{2}-\omega^{2}\right)+i \alpha_{m} \omega} \tilde{\mathbf{H}}(\omega),
\end{aligned}
$$




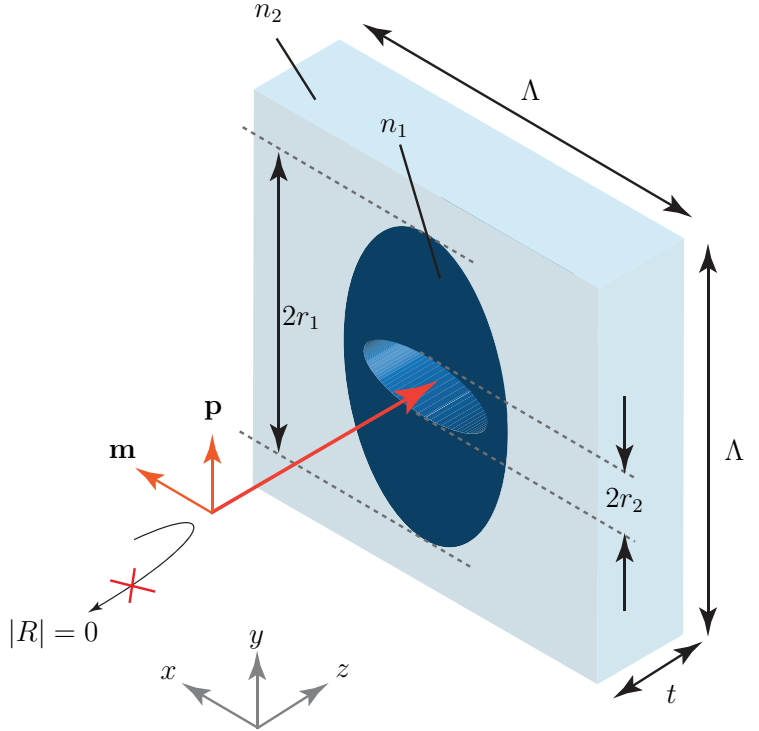

(a)

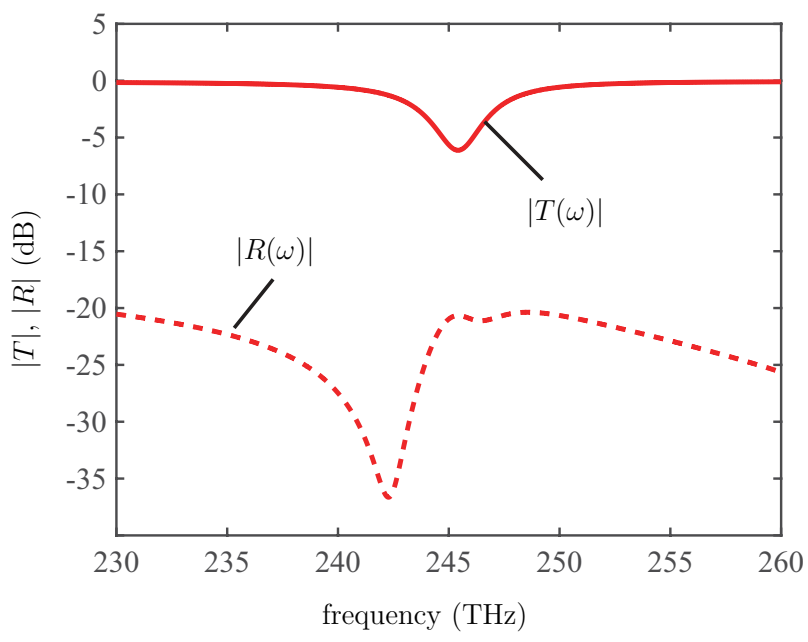

(b)

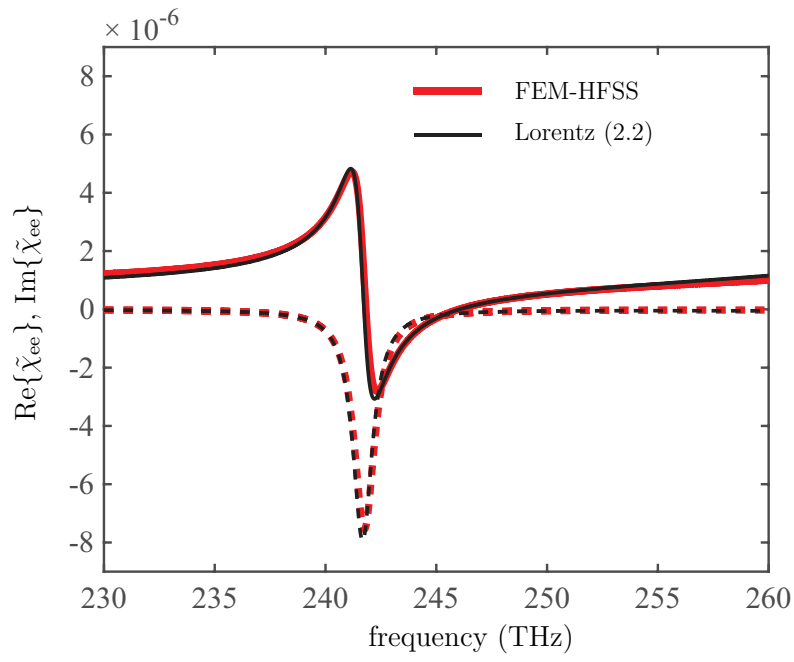

(c)

Figure 2.2 Huygens' source metasurface based on all-dielectric resonators and its constitutive susceptibility parameters. a) A unit cell model with periodicity in both the $x-$ and $y-$ directions. b) Typical amplitude transmission and reflection response, computed using FEMHFSS, when the two dipole moments $\mathbf{p}$ and $\mathbf{m}$ are frequency aligned. c) Extracted electric susceptibility function (magnetic susceptibility not shown for simplicity) from (b) using (2.1) and the Lorentz curve-fit using (2.2). Design parameters: $r_{1}=300 \mathrm{~nm}, t=220 \mathrm{~nm}$, $\Lambda=666 \mathrm{~nm}, n_{2}=1.66$ (Silica) and $n_{1}=3.45$ and $\tan \delta=0.001$ (Silicon). Figure sourced from $[1]$. 
where the susceptibilities are described by a Lorentz frequency response. The frequencydomain responses can be expressed in the time-domain by performing an inverse Fourier transform on the above equations, which leads to

$$
\begin{gathered}
\frac{d^{2} \mathbf{P}(t)}{d t^{2}}+\alpha_{e} \frac{d \mathbf{P}(t)}{d t}+\omega_{e 0}^{2} \mathbf{P}(t)=\epsilon_{0} \omega_{e p}^{2} \mathbf{E}(t) \\
\frac{d^{2} \mathbf{M}(t)}{d t^{2}}+\alpha_{m} \frac{d \mathbf{M}(t)}{d t}+\omega_{m 0}^{2} \mathbf{M}(t)=\omega_{m p}^{2} \mathbf{H}(t) .
\end{gathered}
$$

The Huygens' metasurface of Fig. 2.1 assumes that there are three electromagnetic fields interacting with the metasurface, each inducing their own separate polarizations. This is captured by introducing three time-domain polarization functions $\mathbf{P}_{t}, \mathbf{P}_{r}$ and $\mathbf{P}_{0}$ corresponding to transmitted, reflected and incident fields, respectively. These can be used to construct the average polarizabilities, such that

$$
\begin{aligned}
\mathbf{P}_{\|}(t) & =\frac{\mathbf{P}_{0}(t)+\mathbf{P}_{t}(t)+\mathbf{P}_{r}(t)}{2} \\
\mathbf{M}_{\|}(t) & =\frac{\mathbf{M}_{0}(t)+\mathbf{M}_{t}(t)+\mathbf{M}_{r}(t)}{2},
\end{aligned}
$$

where

$$
\begin{aligned}
\mathbf{P}_{i}(t) & =\mathcal{F}^{-1}\left\{\tilde{\mathbf{P}}_{i}(\omega)\right\}=\mathcal{F}^{-1}\left\{\epsilon_{0} \tilde{\chi}_{\mathrm{ee}}(\omega) \tilde{\mathbf{E}}_{i}(\omega)\right\} \\
\mathbf{M}_{i}(t) & =\mathcal{F}^{-1}\left\{\tilde{\mathbf{M}}_{i}(\omega)\right\}=\mathcal{F}^{-1}\left\{\tilde{\chi}_{\mathrm{mm}}(\omega) \tilde{\mathbf{H}}_{i}(\omega)\right\}
\end{aligned}
$$

with $i=0, \mathrm{t}, \mathrm{r}$, for incident, transmitted and reflected fields. If (2.4) is used for each of these polarizabilities, assuming normally propagated plane-wave field solutions, we get

$$
\begin{gathered}
\frac{d^{2} P_{i}}{d t^{2}}+\alpha_{e} \frac{d P_{i}}{d t}+\omega_{e 0}^{2} P_{i}=\epsilon_{0} \omega_{e p}^{2} E_{i}(t) \\
\frac{d^{2} M_{i}}{d t^{2}}+\alpha_{m} \frac{d M_{i}}{d t}+\omega_{m 0}^{2} M_{i}=\frac{\omega_{m p}^{2}}{\eta_{0}} E_{i}(t)^{1} .
\end{gathered}
$$

Next, substituting (2.5) into (1.2), we get

\footnotetext{
${ }^{1}$ The sign of the right-hand-side expressions is negative $(-)$ for incident fields and transmitted fields, and positive + for reflected fields, following the field conventions of Fig. 2.1
} 


$$
\begin{gathered}
\frac{d M_{t}}{d t}+\frac{d M_{r}}{d t}+\frac{d M_{0}}{d t}=\frac{2}{\mu_{0}}\left(E_{t}-E_{0}-E_{r}\right) \\
\frac{d P_{t}}{d t}+\frac{d P_{r}}{d t}+\frac{d P_{0}}{d t}=2\left(-\frac{E_{t}}{\eta_{0}}+\frac{E_{0}}{\eta_{0}}-\frac{E_{r}}{\eta_{0}}\right) .
\end{gathered}
$$

Equations (2.7) and (2.8) represent a total of eight field equations that must be solved for two primary unknowns, $E_{t}$ and $E_{r}$, and six auxiliary unknowns, $P_{t}, P_{r}, P_{0}, M_{t}, M_{r}$ and $M_{0}$. If a given input excitation field $E_{0}$ is known, these equations can be solved in order to determine the transmitted and reflected fields.

\subsubsection{Finite Difference Formulation}

\section{Assembly of the 1st Order System Equations}

In order to compute the transmitted and reflected fields, the second order differential equations of (2.4) have to be solved for the electric and magnetic response of the metasurface. In traditional MNA circuit solvers, it is inefficient to directly solve higher-ordered differential equations. Therefore, these equations are converted into two first ordered differential equations by introducing two auxiliary variables, $\bar{P}$ and $\bar{M}$. These are defined as

$$
\begin{gathered}
\bar{P}=\frac{1}{\omega_{e 0}} \frac{d P}{d t}+\frac{\alpha_{e}}{\omega_{e 0}} P \\
\bar{M}=\frac{1}{\omega_{m 0}} \frac{d M}{d t}+\frac{\alpha_{m}}{\omega_{m 0}} M .
\end{gathered}
$$

These definitions are chosen due to their equivalent circuit representations, which will be discussed in-depth in the next sub-section. Next, using (2.9) in (2.4), results in

$$
\begin{gathered}
\frac{1}{\omega_{e 0}} \frac{d \bar{P}}{d t}=\frac{\omega_{e p}^{2}}{\omega_{e 0}^{2}} E-P \\
\frac{1}{\omega_{m 0}} \frac{d \bar{M}}{d t}=\frac{\omega_{m p}^{2}}{\eta_{0} \omega_{m 0}^{2}} E-M
\end{gathered}
$$

where it is assumed that the fields on both sides of the metasurface regions are plane-waves, such that $H=E / \eta_{0}$. The general Eqs. (2.9) and (2.10), can now be written in a compact matrix form for the incident field so that 


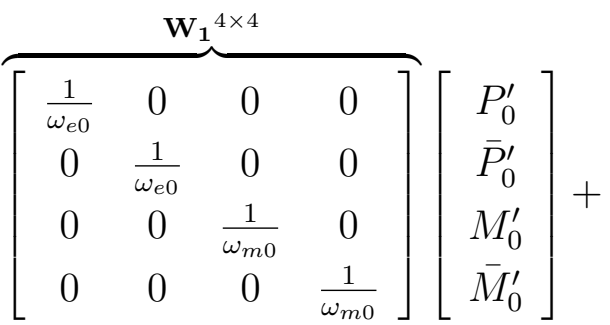

$$
\begin{aligned}
& \overbrace{\left[\begin{array}{cccc}
\frac{\alpha_{e}}{\omega_{e 0}} & -1 & 0 & 0 \\
1 & 0 & 0 & 0 \\
0 & 0 & \frac{\alpha_{m}}{\omega_{m 0}} & -1 \\
0 & 0 & 1 & 0
\end{array}\right]}^{\mathbf{W}_{\mathbf{2}}{ }^{4 \times 4}}\left[\begin{array}{c}
P_{0} \\
\bar{P}_{0} \\
M_{0} \\
\bar{M}_{0}
\end{array}\right]=\overbrace{\left[\begin{array}{c}
0 \\
\frac{\omega_{e p}^{2}}{\omega_{e 0}^{2}} E_{0}(t) \\
0 \\
-\frac{\omega_{m p}^{2}}{\eta_{0} \omega_{m 0}^{2}} E_{0}(t)
\end{array}\right]}^{\mathbf{E}_{\mathbf{1}}}=
\end{aligned}
$$

where the incident field $E_{0}$ terms are kept on the right-hand side of the matrix equation and the primed variables are the time derivatives. Similarly, Eqs. (2.9) and (2.10) are written in matrix form for both the transmitted and reflected fields as

$$
\begin{aligned}
& \overbrace{\left[\begin{array}{ccccc}
\frac{1}{\omega_{e 0}} & 0 & 0 & 0 & 0 \\
0 & \frac{1}{\omega_{e 0}} & 0 & 0 & 0 \\
0 & 0 & \frac{1}{\omega_{m 0}} & 0 & 0 \\
0 & 0 & 0 & \frac{1}{\omega_{m 0}} & 0
\end{array}\right]}^{\mathbf{T}_{1}{ }^{4 \times 5}}\left[\begin{array}{c}
P_{t, r}^{\prime} \\
\bar{P}_{t, r}^{\prime} \\
M_{t, r}^{\prime} \\
\bar{M}_{t, r}^{\prime} \\
E_{t, r}^{\prime}
\end{array}\right]+ \\
& \overbrace{\left[\begin{array}{ccccc}
\frac{\alpha_{e}}{\omega_{e 0}} & -1 & 0 & 0 & 0 \\
1 & 0 & 0 & 0 & -\frac{\omega_{e p}^{2}}{\omega_{e 0}^{2}} \\
0 & 0 & \frac{\alpha_{m}}{\omega_{m 0}} & -1 & 0 \\
0 & 0 & 1 & 0 & \pm \frac{\omega_{m p}^{2}}{\eta_{0} \omega_{m 0}^{2}}
\end{array}\right]}^{\mathbf{T}_{2}^{4 \times 5}}\left[\begin{array}{c}
P_{t, r} \\
\bar{P}_{t, r} \\
M_{t, r} \\
\bar{M}_{t, r} \\
E_{t, r}
\end{array}\right]=\left[\begin{array}{l}
0 \\
0 \\
0 \\
0
\end{array}\right] .
\end{aligned}
$$

Next, the two equations from (2.8) can be expressed in terms of newly introduced auxiliary variables, transforming them into a pair of linear equations, given by

$$
\begin{aligned}
\left(\omega_{m 0} \bar{M}_{t}-\alpha M_{t}\right) & +\left(\omega_{m 0} \bar{M}_{r}-\alpha M_{r}\right)+\left(\omega_{m 0} \bar{M}_{0}-\alpha M_{0}\right) \\
& =\frac{2}{\mu_{0}}\left(E_{t}-E_{r}-E_{0}\right)
\end{aligned}
$$




$$
\begin{aligned}
\left(\omega_{e 0} \bar{P}_{t}-\alpha P_{t}\right) & +\left(\omega_{e 0} \bar{P}_{r}-\alpha P_{r}\right)+\left(\omega_{e 0} \bar{P}_{0}-\alpha P_{0}\right) \\
& =\frac{2}{\eta_{0}}\left(-E_{t}+E_{0}-E_{r}\right)
\end{aligned}
$$

which can then be written in a compact matrix form as

$$
\left[\begin{array}{ccc}
\mathbf{A}_{\mathbf{1}} & \mathbf{A}_{\mathbf{2}} & \mathbf{A}_{\mathbf{3}} \\
\mathbf{B}_{\mathbf{1}} & \mathbf{B}_{\mathbf{2}} & \mathbf{B}_{\mathbf{3}}
\end{array}\right]_{2 \times 14}[\mathbf{V}]_{14 \times 1}=\overbrace{\left[\begin{array}{c}
-\left(2 / \mu_{0}\right) E_{0}(t) \\
\left(2 / \eta_{0}\right) E_{0}(t)
\end{array}\right]}^{\mathbf{E}_{\mathbf{2}}}
$$

where $\mathbf{V}$ is a vector consisting of all the primary and auxiliary unknown variables, i.e.

$$
\begin{array}{r}
{[\mathbf{V}]=\left[P_{0}, \bar{P}_{0}, M_{0}, \bar{M}_{0}, P_{t}, \bar{P}_{t}, M_{t}, \bar{M}_{t}, E_{t}, \ldots\right.} \\
\left.P_{r}, \bar{P}_{r}, M_{r}, \bar{M}_{r} E_{r}\right]^{\top}
\end{array}
$$

with $[\cdot]^{\top}$ is the matrix transpose. Finally combining $(2.11),(2.12)$ and (2.14) in a single matrix, we get

$$
\overbrace{\left[\begin{array}{ccc}
\mathbf{0} & \mathbf{0} & \mathbf{0} \\
\mathbf{0} & \mathbf{0} & \mathbf{0} \\
\mathbf{W}_{1} & \mathbf{0} & \mathbf{0} \\
\mathbf{0} & \mathbf{T}_{1} & \mathbf{0} \\
\mathbf{0} & \mathbf{0} & \mathbf{T}_{1}
\end{array}\right]}^{[\mathrm{C}]} \frac{d[\mathbf{V}]}{d t}+\overbrace{\left[\begin{array}{ccc}
\mathbf{A}_{1} & \mathbf{A}_{2} & \mathbf{A}_{3} \\
\mathbf{B}_{1} & \mathbf{B}_{2} & \mathbf{B}_{3} \\
\mathbf{W}_{2} & \mathbf{0} & \mathbf{0} \\
\mathbf{0} & \mathbf{T}_{2} & \mathbf{0} \\
\mathbf{0} & \mathbf{0} & \mathbf{T}_{2}
\end{array}\right]}^{[\mathbf{G}]}[\mathbf{V}]=[\mathbf{E}]
$$

where

$$
[\mathbf{E}]=\left[\left[\mathbf{E}_{\mathbf{2}}\right]^{\top},\left[\mathbf{E}_{\mathbf{1}}\right]^{\top},[\mathbf{0}]_{[8 \times 1]}^{\top}\right]^{\top}
$$

The resulting $1^{\text {st }}$ order matrix differential equation in time, is given by

$$
[\mathbf{C}] \frac{d[\mathbf{V}]}{d t}+[\mathbf{G}][\mathbf{V}]=[\mathbf{E}]
$$


which can also be expressed equivalently in the frequency domain as

$$
[\mathbf{V}(\omega)]=(j \omega[\mathbf{C}]+[\mathbf{G}])^{-1}[\mathbf{E}(\omega)]
$$

Solving the matrices using standard finite-difference techniques provides the transmitted and reflected fields. While the formulation here only shows a single Lorentz oscillator for simplicity, it is simple to extend this for an arbitrary number of Lorentz contributions, such as in (2.2).

\section{Circuit representation}

The set of equations described by (2.18) is the standard form used widely for circuit analysis. Both the field equations (2.8) and the Lorentzian material behaviour (2.7) are easily represented by circuits. Specifically, the polarization responses (2.7) can be simply seen as a set of coupled RLC oscillators. Fig. 2.3 shows two circuits that represent the response of $M$ and $P$ due to the incident field $E_{0}$, where the corresponding circuit equations in matrix form are given by

$$
\begin{aligned}
& {\left[\begin{array}{cccc}
C_{p} & 0 & 0 & 0 \\
0 & L_{p} & 0 & 0 \\
0 & 0 & C_{m} & 0 \\
0 & 0 & 0 & L_{m}
\end{array}\right]\left[\begin{array}{c}
V_{p 0}^{\prime} \\
I_{p 0}^{\prime} \\
V_{m 0}^{\prime} \\
I_{m 0}^{\prime}
\end{array}\right]+} \\
& {\left[\begin{array}{cccc}
\frac{1}{R_{e}} & -1 & 0 & 0 \\
1 & 0 & 0 & 0 \\
0 & 0 & \frac{1}{R_{m}} & -1 \\
0 & 0 & 1 & 0
\end{array}\right]\left[\begin{array}{c}
V_{p 0} \\
I_{p 0} \\
V_{m 0} \\
I_{m 0}
\end{array}\right]=\left[\begin{array}{c}
0 \\
\frac{\omega_{e p}^{2}}{\omega_{e 0}^{2}} \\
0 \\
-\frac{\omega_{m p}^{2}}{\omega_{m 0}^{2}} \frac{1}{\eta_{0}}
\end{array}\right] E_{0},}
\end{aligned}
$$

where the unknowns are the currents through the inductors and the output voltages. If we identify $V_{p 0} \leftrightarrow P_{0}, V_{m 0} \leftrightarrow M_{0}, I_{p 0} \leftrightarrow \bar{P}_{0}$ and $I_{m 0} \leftrightarrow \eta_{0} \bar{M}_{0}$ and set $L=C=1 / \omega_{0}$ and $R=\frac{\omega_{0}}{\alpha}$, we obtain the matrices derived previously, for example, (2.11). The other polarizations $\left(P_{t}\right.$, $P_{r}, M_{t}$ and $M_{r}$ ) have identical circuit implementations. The field matrix equations are also easily implemented using circuits and the form of (2.18) is then obtained by using standard circuit modelling techniques. This equation can now be solved in either the frequency domain (if not time varying) or it can be solved using a finite-difference formulation using standard techniques for the general case. 


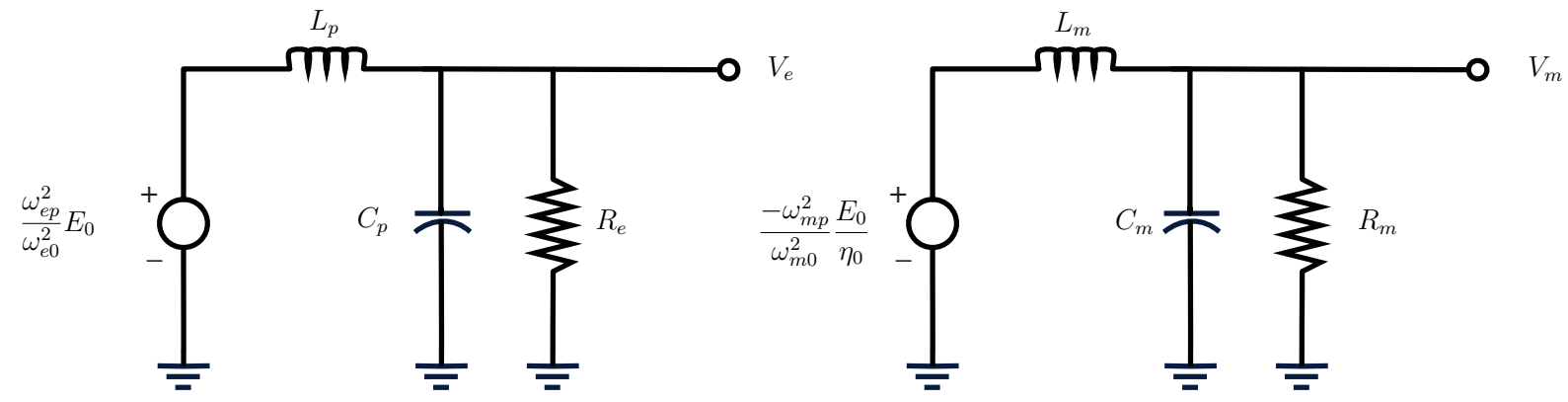

Figure 2.3 A coupled RLC resonator circuit model as an equivalent circuit model representation of the Huygens' metasurface of Fig. 2.2. Figure sourced from [1].

\subsubsection{Extension for Time Varying Metasurface Parameters}

The previous analysis implicitly assumed that the surface parameters are static. Because modifying the permittivity of the surface modifies the parameters of the Lorentzians, which relate $\mathbf{M}$ and $\mathbf{P}$ to the $\mathbf{E}$ fields, an active surface can be easily modelled with a simple extention. This was achieved by modifying the RLC description of the circuit. Because the capacitance $C$ is used to modify the resonant frequency of the Lorentzian, which is the parameter that is being varied in this formulation, a time-varying capacitor is used instead of a static capacitor. This capacitor is described by the equations: $Q_{c}=C(t) V_{c}$ and $I_{c}=d Q / d t$, where $Q_{c}$ is the charge stored in the capacitor, $V_{c}$ is the voltage across the capacitor and $I_{c}$ the current through the capacitor. Because of this, the circuit equations require a minor modification, as shown in (2.23).

This results in a introduction of a new non-linear term to the system equations leading to

$$
[\mathbf{C}] \frac{d[\mathbf{V}]}{d t}+[\mathbf{G}][\mathbf{V}]=[\mathbf{B}(\mathbf{V})]+[\mathbf{E}]
$$

Finally, writing the explicit finite-difference form of (2.21) using the Trapezoidal rule, we get

$$
\begin{aligned}
{[\mathbf{V}]_{\mathbf{i}} } & =\left([\mathbf{C}]+\frac{\Delta t[\mathbf{G}]}{2}\right)^{-1} \\
& \left([\mathbf{C}][\mathbf{V}]_{\mathbf{i}-\mathbf{1}}-\frac{\Delta t[\mathbf{G}][\mathbf{V}]_{\mathbf{i}-\mathbf{1}}}{2}\right. \\
& \left.+\Delta t\left[\frac{[\mathbf{E}]_{\mathbf{i}}+[\mathbf{E}]_{\mathbf{i}-\mathbf{1}}}{2}+\frac{[\mathbf{B}]_{\mathbf{i}}+[\mathbf{B}]_{\mathbf{i}-\mathbf{1}}}{2}\right]\right)
\end{aligned}
$$

where $i$ is the index denoting the current time stamp. 


$$
\left.\begin{array}{c}
{\left[\begin{array}{cccccc}
0 & 0 & 1 & 0 & 0 & 0 \\
0 & L_{p} & 0 & 0 & 0 & 0 \\
0 & 0 & 0 & 0 & 0 & 0 \\
0 & 0 & 0 & 0 & 0 & 1 \\
0 & 0 & 0 & L_{m} & 0 & 0 \\
0 & 0 & 0 & 0 & 0 & 0
\end{array}\right]\left[\begin{array}{c}
V_{p 0}^{\prime} \\
I_{p 0}^{\prime} \\
Q_{p 0}^{\prime} \\
V_{m 0}^{\prime} \\
I_{m 0}^{\prime} \\
Q_{m 0}^{\prime}
\end{array}\right]+\left[\begin{array}{cccccc}
\frac{1}{R_{e}} & -1 & 0 & 0 & 0 & 0 \\
1 & 0 & 0 & 0 & 0 & 0 \\
0 & 0 & 1 & 0 & 0 & 0 \\
0 & 0 & 0 & \frac{1}{R_{m}} & -1 & 0 \\
0 & 0 & 1 & 0 & 0 & 0 \\
0 & 0 & 0 & 0 & 0 & 1
\end{array}\right]\left[\begin{array}{c}
V_{p 0} \\
I_{p 0} \\
Q_{p 0} \\
V_{m 0} \\
I_{m 0} \\
Q_{p 0}
\end{array}\right]=} \\
\\
{\left[\begin{array}{c}
0 \\
0 \\
C_{p}(t) V_{p 0} \\
0 \\
0 \\
C_{m}(t) V_{m 0}
\end{array}\right]+\left[\begin{array}{c}
\omega_{e p}^{2} \\
\omega_{e 0}^{2} \\
0 \\
0 \\
-\frac{\omega_{m p}^{2}}{\omega_{m 0}^{2}} \\
0
\end{array}\right] E_{0}}
\end{array}\right]
$$

\subsection{Propagation Modelling}

\subsubsection{Surface as Boundary}

The explicit metasurface formulation applies a known input wave to the metasurface and calculates the respective transmitted and reflected waves. If there are no scatterers in the region of interest, it can be assumed that the transmitted and reflected waves do not interact with each other. Therefore the propagation of the two waves do not need to be simulated in the same computational region.

One configuration to model the propagation from the surface is shown in Fig. 2.4. Two separate regions are defined in which the EM propagation is modeled either using a standard 2D Yee cell model [26] or by using Fourier propagation techniques [24]. The metasurface is placed at $z=0$ and a region modeling the reflected wave is situated on the left. The second region models the propagation of the transmitted field and it it situated on the right side of the metasurface. The metasurface model is used as a hard boundary source for the two regions. The transmitted field $\mathbf{H}_{t}$ is imposed on the left side of the transmission region at $z=0_{+}$. Similarly, the reflected field $\mathbf{H}_{r}$ is imposed on the right side of the reflection region at $z=0_{-}$.

In the case that the Yee cell is used, the other boundaries of the simulation regions have to be set. To ensure that there are no reflections from the waves, the right-end boundary of the transmission region and the left-end boundary of the reflection region have perfectly matched layers (PMLs). The top and bottom of both regions also have PMLs by default. In the case of modeling an infinite periodic metasurface, the top and bottom PMLs can be 


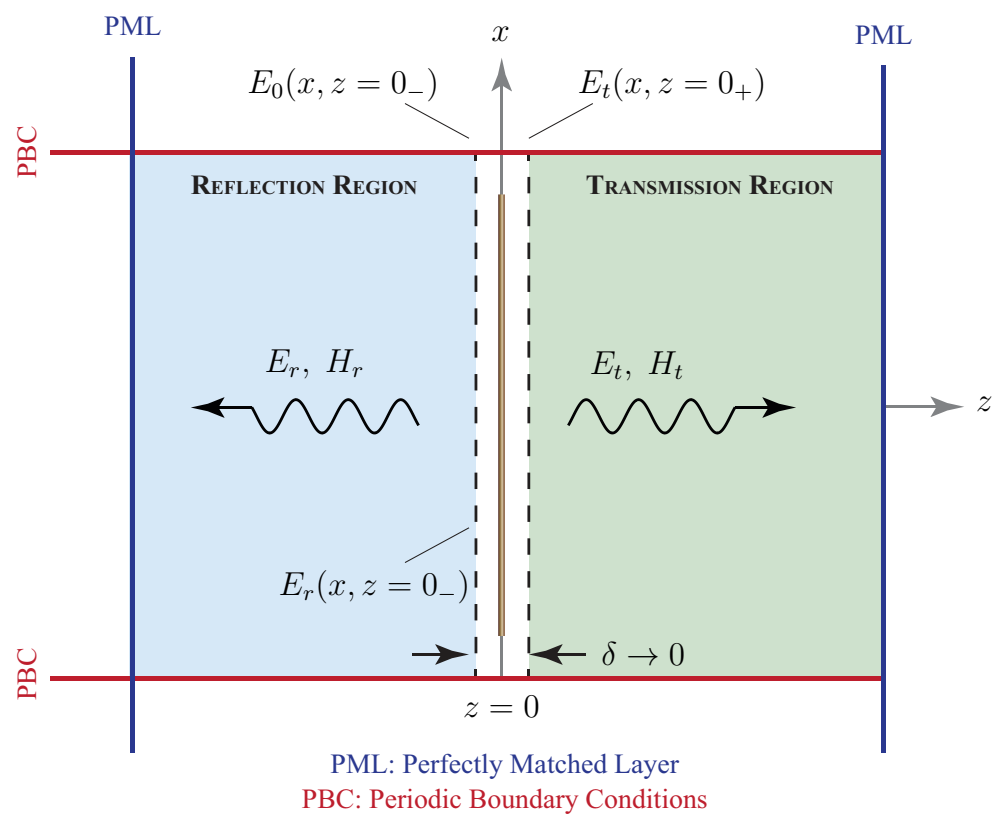

Figure 2.4 Simulation setup for the finite-difference technique showing the numerical domain and the surrounding boundary conditions. Shaded regions on the left and right represent the 2D discretized region where Maxwell's equations are solved on a conventional Yee cell. Figure sourced from [1].

replaced with periodic boundaries, as illustrated in Fig. 2.4.

\subsubsection{Fourier Propagation for Single Frequency}

Fourier propagation is a useful tool for modeling the dispersion of a monochromatic electromagnetic wave through space in the frequency domain [24]. In the case of a source in the time domain that has reached a steady state the source can be converted to the frequency domain by performing a Fourier transform at the given frequency. By taking the magnitude and relative phase of the source the scattered field can be calculated without having to do a time intensive simulation.

For a two dimensional simulation in the $x-z$ plane it is assumed that the source is distributed along the $x$-axis with a value of $\mathbf{E}(x, z=0, t) \hat{\mathbf{y}}$ and a spatial discritization of $\Delta x$ along the source. The temporal field can be expressed in the frequency domain by using a Fast-Fourier Transform (FFT) at each point of the field, leading to $\tilde{\mathbf{E}}(x, z=0, \omega) \hat{\mathbf{y}}$, where $\omega$ is the frequency of the signal.

A spatial FFT is performed on the spatial profile of the temporal frequency which provides the strength of the spatial frequencies $k_{x}$. For each point along $z$ a new spatial FFT 
is calculated using the analytical Fourier propagation method outlined in [24]:

$$
\begin{gathered}
\mathcal{F}_{x}\left\{\tilde{\mathbf{E}}_{t}(x, z, \omega)\right\}=\mathcal{F}_{x}\left\{\tilde{\mathbf{E}}_{t}(x, z=0, \omega)\right\} e^{-j k_{z} z} \\
\text { where } k_{z}=\sqrt{k_{0}^{2}-k_{x}^{2}},
\end{gathered}
$$

with $k_{0}=\omega / c$ being the wavenumber of the given frequency in free-space and $c$ is the speed of light. An inverse FFT is then performed on the new spatial FFT to obtain the magnitude of the scattered steady-state field along $x$ at the $z$ location.

$$
\tilde{\mathbf{E}}_{t}(x, z, \omega)=\mathcal{F}_{x}^{-1}\left\{\mathcal{F}_{x}\left\{\tilde{\mathbf{E}}_{t}(x, z=0, \omega)\right\} e^{-j k_{z} z}\right\} \hat{\mathbf{y}}
$$

Furthermore, the dominant angle of refraction can be calculated using the location of the peak power in the spatial FFT. This is given by:

$$
\theta=\sin ^{-1}\left(\frac{k_{x, \max }}{k_{0}}\right)
$$

where $\theta_{n}$ is the angle of refraction measured from the normal (i.e. $\left.+z\right)$, and $k_{x, \max }$ is the spatial frequency corresponding to the peak value of $\tilde{\mathbf{E}}_{t}\left(k_{x}, z, \omega\right)$.

If the transmitted field $\mathbf{E}_{t}(x, z=0, t) \hat{\mathbf{y}}$ is not monochromatic but is instead broadband at steady state it is still possible to extract the response of each of the individual frequencies. For each $\omega$ the frequency domain signal $\tilde{\mathbf{E}}_{t}(x, z=0, \omega) \hat{\mathbf{y}}$ can be extracted from the FFT and then used in Equation 2.26 to calculate the scattered field.

\subsubsection{D Yee Cell formulation}

The Finite-difference time-domain (FDTD) method, otherwise known as the Yee cell method, is a formulation that is used to propagate an electromagnetic field through space in the time domain [26]. When viewing Maxwell's equations it is observed that the derivative of the electric field in time is dependent on the curl of the magnetic field across space. As a result, solving for the updated electric field in time requires the previously stored electric field and the curl of the local magnetic field distribution in space.

The magnetic field holds a similar relationship. The derivative of the magnetic field in time is dependent on the curl of the electric field across space, and solving for the updated 
magnetic field in time uses the previously stored magnetic field and the curl of the local electric field distribution in space. By continuously updating the electric and magnetic fields in this manner the fields are marched forward through time, resulting in the propagation of the electromagnetic wave through the simulated region.

This leads to the modern Yee cell lattice, which spatially staggers the electric and magnetic field's vector components about rectangular unit cells in the Cartesian coordinates. The field updates are further staggered in time so that the numerical calculations of the electric field are performed between the calculations of the magnetic field, and vice-versa. This formulation gives explicit time-stepping equations that are centered in both space and time, which proves to be both accurate and time efficient.

Fig. 2.5 shows an example of a 2D Yee cell lattice in the $x-z$ plane. If it is assumed that a TE mode wave is propagating through the region, the field components $E_{y}, H_{x}$ and $H_{z}$ are used to set up the Yee cell and the rest of the field components are zero. From this assumption, the update equations can be extracted from Maxwell's equations for a nondispersive medium, which are shown below.

$$
\begin{gathered}
\frac{\partial H_{x}}{d t}=\frac{1}{\mu}\left(\frac{\partial E_{y}}{\partial z}\right) \\
\frac{\partial H_{z}}{d t}=\frac{1}{\mu}\left(-\frac{\partial E_{y}}{\partial x}\right) \\
\frac{\partial E_{y}}{d t}=\frac{1}{\epsilon}\left(\frac{\partial H_{x}}{\partial z}-\frac{\partial H_{z}}{\partial x}\right)
\end{gathered}
$$

Equations 2.28 - 2.30 coupled together with the Yee cell discretization and the staggered time-stepping yield the proper update equations for the fields as well as the update order for the time step in question.

At time $t=(n+1 / 2) \Delta t$ in the simulation the magnetic fields $H_{x}$ and $H_{z}$ are updated using a central difference in time at $n$ :

$$
\begin{aligned}
& \left.H_{x}\right|_{i+1 / 2, j} ^{n+1 / 2}=\left.H_{x}\right|_{i+1 / 2, j} ^{n-1 / 2}+\frac{\Delta t}{\mu}\left(\frac{\left.E_{y}\right|_{i+1, j} ^{n}-\left.E_{y}\right|_{i, j} ^{n}}{\Delta z}\right) \\
& \left.H_{z}\right|_{i, j+1 / 2} ^{n+1 / 2}=\left.H_{z}\right|_{i, j+1 / 2} ^{n-1 / 2}-\frac{\Delta t}{\mu}\left(\frac{\left.E_{y}\right|_{i, j+1} ^{n}-\left.E_{y}\right|_{i, j} ^{n}}{\Delta x}\right)
\end{aligned}
$$

where $n$ is the current index in time, $\Delta t$ is the discritization in time, $i$ and $j$ are the spatial indexes in the $z$ and $x$ directions respectively, and $\Delta z$ and $\Delta x$ are the spatial separations 


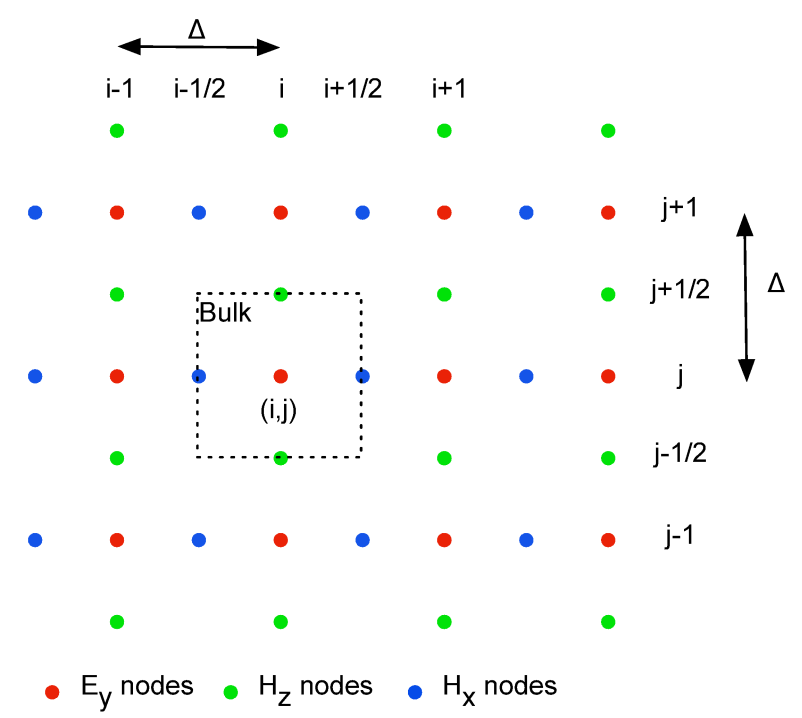

Figure 2.5 Yee cell lattice in $2 \mathrm{D}$ with $E_{y}, H_{x}$ and $H_{z}$ fields. $i$ is the index in space along the $z$ direction, $j$ is the index in space along the $x$ direction, and $\Delta$ is the spatial distance between two field nodes.

between field nodes in the $z$ and $x$ directions respectively. After the magnetic fields are updated, the electric field $E_{y}$ is updated at time $t=(n+1) \Delta t$ using a central difference in time at $n+1 / 2$.

$$
\left.E_{y}\right|_{i, j} ^{n+1}=\left.E_{y}\right|_{i, j} ^{n}+\frac{\Delta t}{\mu}\left(\frac{\left.H_{x}\right|_{i+1 / 2, j} ^{n+1 / 2}-\left.H_{x}\right|_{i-1 / 2, j} ^{n+1 / 2}}{\Delta z}-\frac{\left.H_{z}\right|_{i, j+1 / 2} ^{n+1 / 2}-\left.H_{z}\right|_{i, j-1 / 2} ^{n+1 / 2}}{\Delta x}\right)
$$

Once all of the fields are updated, the time of the simulation is incremented and the steps are repeated for the next value of $n$. 


\section{Chapter 3 Method and Formulation}

This chapter contains the theoretical and implementation work that is required to model an all-dielectric metasurface with space-time modulation. This material has been presented in my paper which is currently under review [2]. The method to extract the surface susceptibilities from an example all-dielectric unit cell, as well as how the constitutive parameters of the susceptibilities vary as a function of the cell's permittivity, is discussed in detail. The governing field equations for an equivalent zero-thickness metasurface are then presented, which models the metasurface with the extracted Lorentzian susceptibilities, and discusses the steps that were used to develop the FDTD formulation.

The metasurface is then presented as an internal boundary inside of a conventional Yeecell. The steps to model the metasurface with a general space-time modulation to capture the transmitted and reflected fields in the time domain are then outlined. The techniques to analyze the results of a simulation using spatial and temporal Fourier transform analysis, which was originally presented in my conference paper [3], are also developed and explained in detail.

\subsection{Susceptibility Extraction}

Before a time-varying Huygens' surface can be modelled, its constitutive susceptibility functions must first be determined. A Huygen's metasurface is typically implemented using sub-wavelength all-dielectric unit cell sources. Fig. 3.1(a) shows one unit cell configuration, consisting of a holey dielectric resonator structure $\left(\epsilon_{r}\right)$ embedded in a host medium $\left(\epsilon_{h}\right)$. The unit cell size $\Lambda_{x}=\Lambda_{y}<\lambda_{0}$ to ensure the existence of a zeroth diffraction order only. The dielectric resonator is geometrically described in terms of its ellipticity $(\tau)$, thickness $(h)$ and the outer $\left(d_{1}\right)$ and inner diameters $\left(d_{2}\right)$ of the structure. By controlling the geometrical parameters, the Huygens' unit cell can be designed to achieve a good transmission in a wide bandwidth while also minimizing the reflection in the same range. A convenient way to emulate a space-time modulated all-dielectric metasurface is by varying the permittivity, $\epsilon_{r}$, of the dielectric resonator, so that

$$
\epsilon_{r}(x, t)=\epsilon_{r}\{1+\Delta_{p} \overbrace{\sin \left(\omega_{p} t-\beta_{p} x\right)}^{f_{m}(t)}\},
$$

where $\omega_{p}$ is called the pumping frequency, $\beta_{p}$ is the spatial frequency of the perturbation in 
the permittivity and $\Delta_{p}$ is the modulation index.
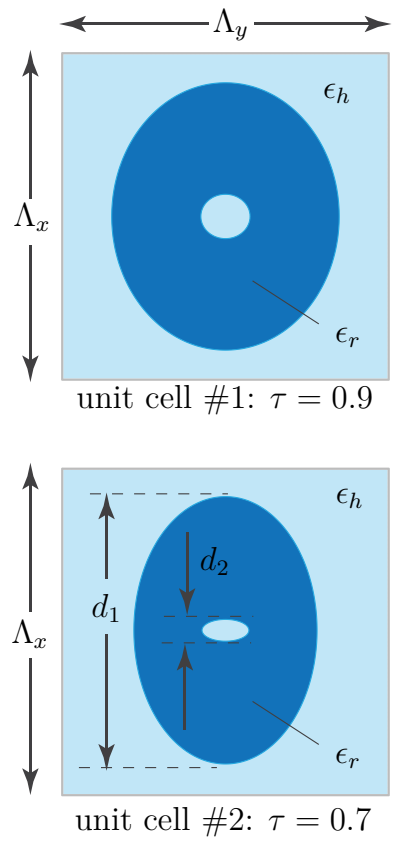

(a)
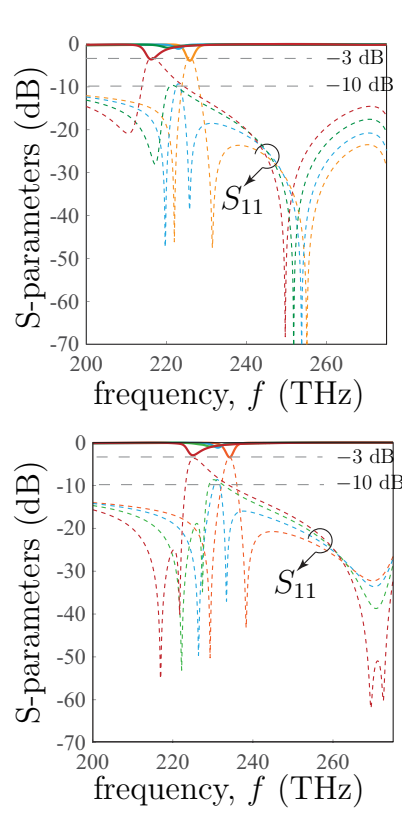

(b)
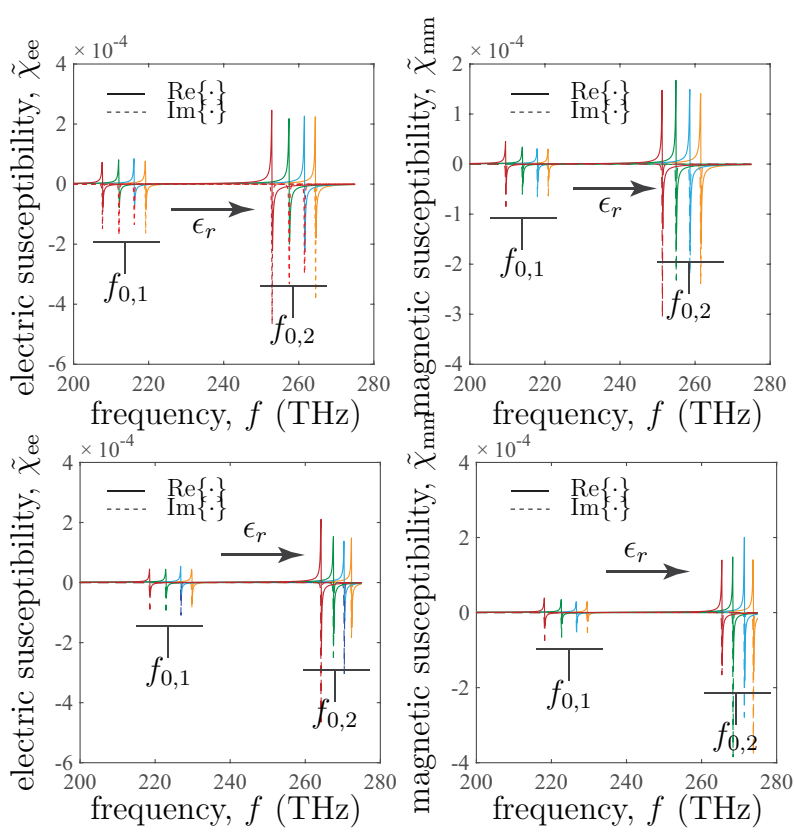

(c)

Figure 3.1 All-dielectric unit cell for different material permittivities, $\epsilon_{r}=$ $(11,11.5,12.2,13)$. a) Two different unit cell geometries showing the transverse view with wave propagation along $z$. b) Transmission and reflection responses for varying permittivity values, and c) the corresponding extracted complex electric and magnetic susceptibilities, $\tilde{\chi}_{\mathrm{ee}}$ and $\tilde{\chi}_{\mathrm{mm}}$, respectively. The full-wave simulation parameters are: unit cell $\# 1-d_{2}=80 \mathrm{~nm}$, unit cell $\# 2-d_{2}=60 \mathrm{~nm}$. $d_{1}=560 \mathrm{~nm}$, substrate thickness $h=220 \mathrm{~nm}$ and $\epsilon_{h}=2.76$ for both cases. Figure sourced from [2].

To determine the effect that the permittivity has on the transmission and reflection responses of the metasurface, which will lead to a good estimate on a $\Delta_{p}$ value, a reference unit cell is first designed with a nominal permittivity value that matches silicon, $\epsilon_{r}=11.9$. At this permittivity, the unit cell is designed so that it has a quasi-perfect transmission through the metasurface in the entire frequency band of interest, or in other words a matched metasurface with $\left|S_{11}\right|<-10 \mathrm{~dB}$. If all other dimensions remain the same, any variation in $\epsilon_{r}$ from this optimal value is expected to increase the reflection from the metasurface. Fig. 3.1(b) shows the FEM-HFSS simulated transmission and reflection responses of two different unit cell designs, for various dielectric permittivities around the nominal value of $\epsilon_{r}=11.9$. It is found that when $11.5<\epsilon_{r}<12.2$, the metasurface still exhibits a good transmission with minimal reflections throughout the band. However, when this range is increased so that $11.50<\epsilon_{r}<13.0$, a strong reflection is observed with a maximum value of $S_{11}=-3 \mathrm{~dB}$. 
This thus gives us two domains of $\Delta_{p} \approx 0.1$ and $\Delta_{p} \approx 0.05$, corresponding to a strong and weakly reflecting regimes.

For linear time-invariant metasurfaces the electric and magnetic surface susceptibilities are related to the total reflected and transmitted fields through the following [6]:

$$
\begin{gathered}
\tilde{\chi}_{\mathrm{ee}}(\omega)=\frac{2 j}{k}\left(\frac{S_{21}+S_{11}-1}{S_{21}+S_{11}+1}\right), \\
\tilde{\chi}_{\mathrm{mm}}(\omega)=\frac{2 j}{k}\left(\frac{S_{21}-S_{11}-1}{S_{21}-S_{11}+1}\right),
\end{gathered}
$$

where $S_{21}(\omega)=\tilde{E}_{t} / \tilde{E}_{i}$ and $S_{11}(\omega)=\tilde{E}_{r} / \tilde{E}_{i}$. By applying (3.2) to the full-wave simulated transmission and reflection responses, the corresponding electric and magnetic susceptibilities are extracted for each unit cell and for different permittivity values, as shown in Fig. 3.1(c). To accurately quantify this behaviour, both $\tilde{\chi}_{\mathrm{ee}}$ and $\tilde{\chi}_{\mathrm{mm}}$, are approximated using a doubleLorentz function following

$$
\begin{gathered}
\tilde{\chi}_{\mathrm{ee}}\left(\omega, \epsilon_{r}\right)=\sum_{i=1}^{2} \frac{\omega_{e p, i}^{2}}{\left(\omega_{e 0, i}^{2}-\omega^{2}\right)+i \alpha_{e, i} \omega} \\
\tilde{\chi}_{\mathrm{mm}}\left(\omega, \epsilon_{r}\right)=\sum_{i=1}^{2} \frac{\omega_{m p, i}^{2}}{\left(\omega_{m 0, i}^{2}-\omega^{2}\right)+i \alpha_{m, i} \omega} .
\end{gathered}
$$

The extracted resonant frequencies of each unit cell as a function of puck's permittivity is shown in Fig. 3.2. It can be seen that both electric and magnetic susceptibilities require two resonant contributions. Furthermore, with decreasing permittivity, these resonances are shifted towards higher frequencies, as expected.

Within this small range of permittivity, the various resonant frequencies are shown to follow $\epsilon_{r}$ in a quasi-linear manner. This leads to an important conclusion: for a time-varying all-dielectric metasurface, any variation of permittivity can be equivalently modelled by varying the parameters (in particular, the resonant frequencies) of the Lorentzian susceptibilities using the same modulation function $f_{m}(t)$, or in other words:

$$
\begin{gathered}
\omega_{e 0}(t)=\omega_{e 0}+f_{e 0}(t)=\omega_{e 0}+\Delta \omega_{e} \sin \left(\omega_{p} t\right) \\
\omega_{m 0}(t)=\omega_{m 0}+f_{m 0}(t)=\omega_{m 0}+\Delta \omega_{m} \sin \left(\omega_{p} t\right),
\end{gathered}
$$

where $\Delta \omega_{e, m}$ can be easily extracted from the slopes of the curves in Fig. $3.2^{1}$. Finally, it

\footnotetext{
${ }^{1}$ The relationship between the resonant frequencies and $\epsilon_{r}$ can also be modelled using a higher ordered
} 
should be noted that, in general, all the parameters of the Lorentzian susceptibilities (plasma frequencies $\omega_{p}$, resonant frequencies $\omega_{0}$ and loss coefficient, $\alpha$ ) depend on the permittivity $\epsilon_{r}$. However, for the units cells considered here, the variations in the plasma frequency and loss coefficient were found to be negligible, compared to that for resonant frequencies, and thus are ignored for this thesis ${ }^{2}$. Consequently, the problem of computing the scattered fields from a time-varying (in general, space-time varying), finite-thickness all-dielectric metasurface for the given incident fields, can now be solved using a zero-thickness metasurface with Lorentzian electric and magnetic susceptibilities with space-time-varying resonant frequencies.

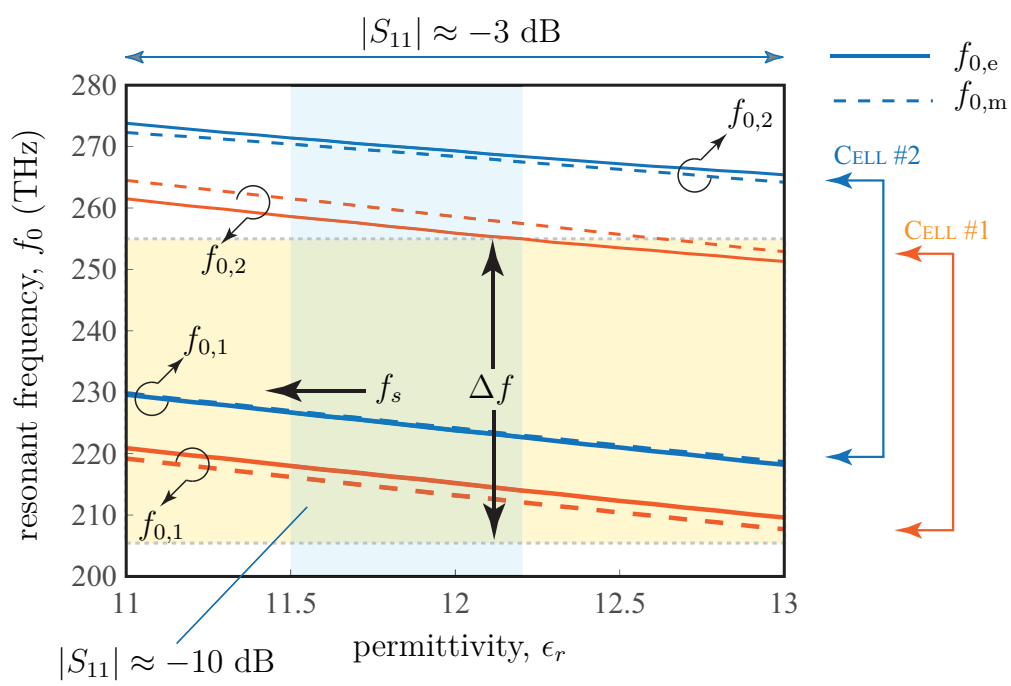

Figure 3.2 The variation of the electric and magnetic resonant frequencies of the three metasurface unit cells of Fig. 3.1, as a function of material permittivity $\epsilon_{r}$, for both the first and second Lorentzian contributions. The center frequency and the bandwidth of the incident signal are $f_{s}=230 \mathrm{THz}$ and $\Delta f \approx 50 \mathrm{THz}$, respectively. Figure sourced from [2].

\subsection{Field Equations and Finite-Difference Formulation}

\subsubsection{Analytical Formulation of Space-Time Modulated Metasurfaces}

Section 2.1.2 describes one of the earlier methods to properly model a time modulated metasurface using a circuit that implemented time-varying capacitors. While that method works very well and properly models the metasurface under a variety of conditions, the simulation

polynomial, such as a quadratic function. However, this did not cause any changes in the results because it is very close to linear.

${ }^{2}$ While these parameters were found to be negligible, and thus kept equal to their normal static values to avoid cumbersome details here, they can be easily taken into account in the formulation at the expense of more complex modelling. 
takes longer to finish. In order to speed up the simulation of the metasurface, a new model was created, which brings it closer to a more analytical representation of the metasurface.

The relationship between the induced polarization densities and the exciting fields is described by (2.3) in the frequency domain, which for a monochromatic excitation, becomes a simple product in time-domain of $\chi$ and the corresponding temporal fields. However, for timeinvariant metasurfaces, the problem is solved in the time-domain and thus the relationship between the polarization densities and the fields must be expressed as convolutions, i.e.

$$
\begin{aligned}
\mathbf{P}_{\|}(t) & =\epsilon_{0} \chi_{\mathrm{ee}}(t) *\left[\frac{\mathbf{E}_{0}(t)+\mathbf{E}_{t}(t)+\mathbf{E}_{r}(t)}{2}\right] \\
\mathbf{M}_{\|}(t) & =\chi_{\mathrm{mm}}(t) *\left[\frac{\mathbf{M}_{0}(t)+\mathbf{M}_{t}(t)+\mathbf{M}_{r}(t)}{2}\right] .
\end{aligned}
$$

Let us express the surface polarizabilities $\mathbf{P}_{\|}$and $\mathbf{M}_{\|}$in terms of average polarizations densities associated with incident, reflected and transmitted fields seperately, such that ${ }^{3}$

$$
\begin{aligned}
\mathbf{P}_{\|}(t) & =\frac{\mathbf{P}_{0}(t)+\mathbf{P}_{t}(t)+\mathbf{P}_{r}(t)}{2} \\
\mathbf{M}_{\|}(t) & =\frac{\mathbf{M}_{0}(t)+\mathbf{M}_{t}(t)+\mathbf{M}_{r}(t)}{2}
\end{aligned}
$$

where

$$
\begin{aligned}
\mathbf{P}_{i}(t) & =\mathcal{F}^{-1}\left\{\tilde{\mathbf{P}}_{i}(\omega)\right\}=\mathcal{F}^{-1}\left\{\epsilon_{0} \tilde{\chi}_{\mathrm{ee}}(\omega) \tilde{\mathbf{E}}_{i}(\omega)\right\} \\
\mathbf{M}_{i}(t) & =\mathcal{F}^{-1}\left\{\tilde{\mathbf{M}}_{i}(\omega)\right\}=\mathcal{F}^{-1}\left\{\tilde{\chi}_{\mathrm{mm}}(\omega) \tilde{\mathbf{H}}_{i}(\omega)\right\}
\end{aligned}
$$

and the subscript $i=0$, $\mathrm{t}$, and $\mathrm{r}$ indicate if it is the incident, transmitted and reflected fields, respectively. Since $\tilde{\chi}_{\mathrm{ee}}$ and $\tilde{\chi}_{\mathrm{mm}}$ exhibit Lorentz distributions following (3.3), these relationships can be expressed in the time domain for time-varying resonant frequencies (and for each Lorentz contribution), as

$$
\frac{d^{2} Q_{i}}{d t^{2}}+\alpha_{e} \frac{d Q_{i}}{d t}+\omega_{e 0}^{2}(t) Q_{i}=\omega_{e p}^{2} E_{i}(t)
$$

\footnotetext{
${ }^{3}$ For simplicity, all physical quantities are described here as function of time $t$ only for notational compactness. Wherever appropriate, they can be treated in general as functions of both space and time.
} 


$$
\frac{d^{2} M_{i}}{d t^{2}}+\alpha_{m} \frac{d M_{i}}{d t}+\omega_{m 0}^{2}(t) M_{i}=\frac{\omega_{m p}^{2}}{\eta_{0}} E_{i}(t),{ }^{4}
$$

$Q_{i}=P_{i} / \epsilon_{0}$. Finally, from (1.2), under plane-wave excitations and assuming normally incident and outgoing plane-waves valid for small divering angles,

$$
\begin{gathered}
\frac{d M_{t}}{d t}+\frac{d M_{r}}{d t}+\frac{d M_{0}}{d t}=\frac{2}{\mu_{0}}\left(E_{t}-E_{r}-E_{0}\right) \\
\frac{d Q_{t}}{d t}+\frac{d Q_{r}}{d t}+\frac{d Q_{0}}{d t}=\frac{2}{\eta_{0} \epsilon_{0}}\left(E_{0}-E_{t}-E_{r}\right) .
\end{gathered}
$$

Equations (3.8) and (3.9), represent a total of eight field equations to be solved for two primary unknowns, $E_{t}, E_{r}$, and six auxiliary unknowns, $P_{t}, P_{r}, P_{0}, M_{t}, M_{r}, M_{0}$, for a given input excitation field $E_{0}$. It should be noted that, while the metasurface susceptibilities exhibit double-Lorentz response, the formulation presented here is shown for only one Lorentzian contribution for simplicity. In reality, (3.8) represents 12 equations, 6 equations each for every Lorentzian term of $\tilde{\chi}$ 's as described in (3.3). The extention to a double-Lorentz response is demonstrated in Appendix A. Finally, the time-varying nature of the metasurface can be modelled using (3.4) for a given modulation function $f_{m}(t)$.

\subsubsection{Finite Difference Formulation}

The above set of equations can be conveniently solved by converting the second-order differential equations of (3.8) into two first-order differential equations. For example, for electric polarization densities associated with incident fields, following (3.8), we get $[1],{ }^{5}$

$$
\begin{gathered}
\omega_{e 0} \bar{Q}_{0}=\frac{d Q_{0}}{d t}+\alpha_{e} Q_{0}, \\
\frac{d \bar{Q}_{0}}{d t}+\bar{Q}_{0} \frac{1}{\omega_{e 0}} \frac{d \omega_{e 0}}{d t}+\omega_{e 0} Q_{0}=\frac{\omega_{e p}^{2}}{\omega_{e 0}} E_{0} \\
\omega_{m 0} \bar{M}_{0}=\frac{d M_{0}}{d t}+\alpha_{m} M_{0}, \\
\frac{d \bar{M}_{0}}{d t}+\bar{M}_{0} \frac{1}{\omega_{m 0}} \frac{d \omega_{m 0}}{d t}+\omega_{m 0} M_{0}=-\frac{\omega_{m p}^{2}}{\eta_{0} \omega_{m 0}} E_{0} .
\end{gathered}
$$

Similar sets of equations can also be written for electric and magnetic polarizabilities, for both transmitted and reflected fields, as given in (3.11), (3.12) and (3.13). These equations

\footnotetext{
${ }^{4}$ with RHS times (-1) for $E_{0}$ and $E_{t}$ and $(+1)$ for $E_{r}$.

${ }^{5}$ The time dependence, $(t)$, is dropped for compact notation.
} 
can be written in a compact matrix form for incident, reflected and transmitted fields as

$$
\begin{aligned}
& \mathbf{W}_{\mathbf{1}}\left[\begin{array}{c}
Q_{0}^{\prime} \\
\bar{Q}_{0}^{\prime} \\
M_{0}^{\prime} \\
\bar{M}_{0}^{\prime}
\end{array}\right]+\mathbf{W}_{\mathbf{2}}(t) \overbrace{\left[\begin{array}{c}
Q_{0} \\
\bar{Q}_{0} \\
M_{0} \\
\bar{M}_{0}
\end{array}\right]}^{\left[\mathbf{V}_{\mathbf{0}}\right]}=\left[\mathbf{E}_{\mathbf{1}}(t)\right] \\
& \mathbf{T}_{\mathbf{1}}\left[\begin{array}{c}
Q_{t}^{\prime} \\
\bar{Q}_{t}^{\prime} \\
M_{t}^{\prime} \\
\bar{M}_{t}^{\prime} \\
E_{t}^{\prime}
\end{array}\right]+\mathbf{T}_{\mathbf{2}}(t) \overbrace{\left[\begin{array}{c}
Q_{t} \\
\bar{Q}_{t} \\
M_{t} \\
\bar{M}_{t} \\
E_{t}
\end{array}\right]}^{\left[\mathbf{V}_{\mathbf{t}}\right]}=[\mathbf{0}], \\
& \mathbf{T}_{\mathbf{1}}\left[\begin{array}{c}
Q_{r}^{\prime} \\
\bar{Q}_{r}^{\prime} \\
M_{r}^{\prime} \\
\bar{M}_{r}^{\prime} \\
E_{r}^{\prime}
\end{array}\right]+\mathbf{T}_{\mathbf{2}}(t) \overbrace{\left[\begin{array}{c}
Q_{r} \\
\bar{Q}_{r} \\
M_{r} \\
\bar{M}_{r} \\
E_{r}
\end{array}\right]}^{\left[\mathbf{V}_{\mathbf{r}}\right]}=[\mathbf{0}],
\end{aligned}
$$

where matrix $\left[\mathbf{E}_{\mathbf{1}}\right]$ includes the known excitation fields specified at the input of the metasurface. Similarly, the GSTC equations of (3.9) can be written in terms of new auxiliary variables as

$$
\begin{gathered}
\left(\omega_{m 0} \bar{M}_{0}-\alpha_{m} M_{0}\right)+\left(\omega_{m 0} \bar{M}_{r}-\alpha_{m} M_{r}\right) \\
+\left(\omega_{m 0} \bar{M}_{t}-\alpha_{m} M_{t}\right)=\frac{2}{\mu_{0}}\left(E_{t}-E_{r}-E_{0}\right), \\
\left(\omega_{e 0} \bar{Q}_{0}-\alpha_{e} Q_{0}\right)+\left(\omega_{e 0} \bar{Q}_{r}-\alpha_{e} Q_{r}\right)+\left(\omega_{e 0} \bar{Q}_{t}-\alpha_{e} Q_{t}\right) \\
=\frac{2}{\epsilon_{0} \eta_{0}}\left(E_{0}-E_{t}-E_{r}\right),
\end{gathered}
$$

to be further written in a compact matrix form as 


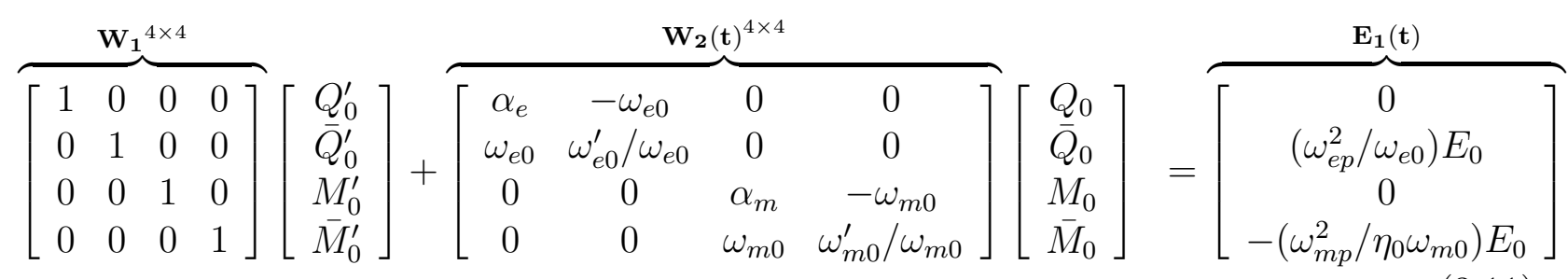

$$
\overbrace{\left[\begin{array}{lllll}
1 & 0 & 0 & 0 & 0 \\
0 & 1 & 0 & 0 & 0 \\
0 & 0 & 1 & 0 & 0 \\
0 & 0 & 0 & 1 & 0
\end{array}\right]}^{\mathbf{T}_{4} \times 5}\left[\begin{array}{c}
Q_{t}^{\prime} \\
\bar{Q}_{t}^{\prime} \\
M_{t}^{\prime} \\
\bar{M}_{t}^{\prime} \\
E_{t}^{\prime}
\end{array}\right]+\overbrace{\left[\begin{array}{ccccc}
\alpha_{e} & -\omega_{e 0} & 0 & 0 & 0 \\
\omega_{e 0} & \omega_{e 0}^{\prime} / \omega_{e 0} & 0 & 0 & -\omega_{e p}^{2} / \omega_{e 0} \\
0 & 0 & \alpha_{m} & -\omega_{m 0} & 0 \\
0 & 0 & \omega_{m 0} & \omega_{m 0}^{\prime} / \omega_{m 0} & \left(\omega_{m p}^{2} / \eta_{0} \omega_{m 0}\right)
\end{array}\right]}^{\mathbf{T}_{2}(\mathbf{t} \mathbf{t})^{4 \times 5}}\left[\begin{array}{c}
Q_{t} \\
\bar{Q}_{t} \\
M_{t} \\
\bar{M}_{t} \\
E_{t}
\end{array}\right]=\overbrace{\left[\begin{array}{l}
0 \\
0 \\
0 \\
0
\end{array}\right]}^{\mathbf{E}_{2}}
$$

$$
\overbrace{\left[\begin{array}{lllll}
1 & 0 & 0 & 0 & 0 \\
0 & 1 & 0 & 0 & 0 \\
0 & 0 & 1 & 0 & 0 \\
0 & 0 & 0 & 1 & 0
\end{array}\right]}^{\mathbf{T}_{1} \times 5}\left[\begin{array}{c}
Q_{r}^{\prime} \\
\bar{Q}_{r}^{\prime} \\
M_{r}^{\prime} \\
\bar{M}_{r}^{\prime} \\
E_{r}^{\prime}
\end{array}\right]+\overbrace{\left[\begin{array}{ccccc}
\alpha_{e} & -\omega_{e 0} & 0 & 0 & 0 \\
\omega_{e 0} & \omega_{e 0}^{\prime} / \omega_{e 0} & 0 & 0 & -\omega_{e p}^{2} / \omega_{e 0} \\
0 & 0 & \alpha_{m} & -\omega_{m 0} & 0 \\
0 & 0 & \omega_{m 0} & \omega_{m 0}^{\prime} / \omega_{m 0} & -\left(\omega_{m p}^{2} / \eta_{0} \omega_{m 0}\right)
\end{array}\right]}^{\mathbf{T}_{3}\left(\mathbf{t} \mathbf{t}^{4 \times 5}\right.}\left[\begin{array}{c}
Q_{r} \\
\bar{Q}_{r} \\
M_{r} \\
\bar{M}_{r} \\
E_{r}
\end{array}\right]=\overbrace{\left[\begin{array}{c}
0 \\
0 \\
0 \\
0
\end{array}\right]}^{\mathbf{E}_{2}}
$$

$$
\left[\begin{array}{lll}
\mathbf{A}_{\mathbf{1}} & \mathbf{A}_{\mathbf{2}} & \mathbf{A}_{\mathbf{3}} \\
\mathbf{B}_{\mathbf{1}} & \mathbf{B}_{\mathbf{2}} & \mathbf{B}_{3}
\end{array}\right]_{2 \times 14}[\mathbf{V}]_{14 \times 1}=\overbrace{\left[\begin{array}{c}
-\left(2 / \mu_{0}\right) E_{0} \\
\left(2 / \epsilon_{0} \eta_{0}\right) E_{0}
\end{array}\right]}^{\mathbf{E}_{3}},
$$

where $\mathbf{V}$ is a vector consisting of all the primary and auxiliary unknown variables, given by $[\mathbf{V}]=\left[V_{0}, V_{t}, V_{r}\right]^{\top}$, with $[\cdot]^{\top}$ as the transpose of the matrix and 


$$
\begin{aligned}
& {\left[\mathbf{A}_{1}\right]=\left[0,0,-\alpha_{m}, \omega_{m 0}\right]} \\
& {\left[\mathbf{A}_{2}\right]=\left[0,0,-\alpha_{m}, \omega_{m 0},-2 / \mu_{0}\right]} \\
& {\left[\mathbf{A}_{3}\right]=\left[0,0,-\alpha_{m}, \omega_{m 0}, 2 / \mu_{0}\right]} \\
& {\left[\mathbf{B}_{1}\right]=\left[-\alpha_{e}, \omega_{e 0}, 0,0\right]} \\
& {\left[\mathbf{B}_{2}\right]=\left[\mathbf{B}_{3}\right]=\left[-\alpha_{e}, \omega_{e 0}, 0,0,2 / \epsilon_{0} \eta_{0}\right]}
\end{aligned}
$$

Finally, using (3.14) and (3.16), we get

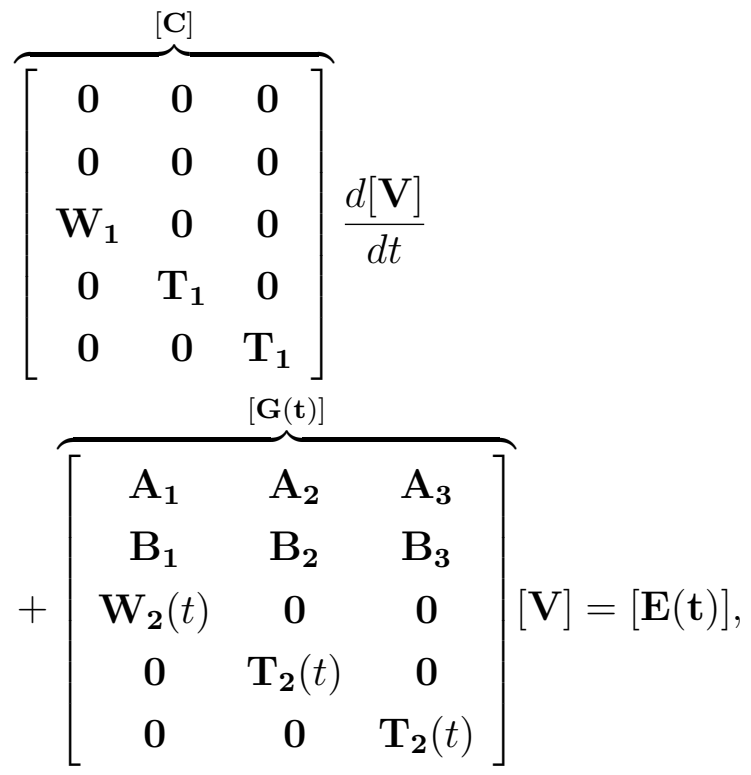

which can then further be written in compact notation as

$$
[\mathbf{C}] \frac{d[\mathbf{V}]}{d t}+[\mathbf{G}(\mathbf{t})][\mathbf{V}]=[\mathbf{E}]
$$

where $[\mathbf{E}]=\left[E_{3}, E_{1}, E_{2}, E_{2}\right]^{\top}$. In summary, for a specified time-domain field $E_{0}(x, t)$, included in $[\mathbf{E}]$ prescribed at the input of the metasurface at $z=0_{-}$, the above matrix equation provides the reflected fields $E_{r}(x, t)$ at $z=0_{-}$and transmitted fields $E_{t}(x, t)$ at $z=0_{+}$, which are both included in the solution matrix $[\mathbf{V}]$. Matrices $[\mathbf{C}]$ and $[\mathbf{G}]$ contains the exact description of a given space-time modulated metasurface. For the trivial case of a static metasurface, $[\mathbf{G}(\mathbf{t})]=[\mathbf{G}]$, so that $[1]$ 


$$
[\mathbf{C}] \frac{d[\mathbf{V}]}{d t}+[\mathbf{G}][\mathbf{V}]=[\mathbf{E}]
$$

Finally, writing the explicit finite-difference form of (3.18) using the trapezoidal rule of integration, we get

$$
\begin{aligned}
{[\mathbf{V}]_{i}=\left([\mathbf{C}]+\frac{\Delta t}{2}[\mathbf{G}]_{i}\right)^{-1} } & {\left[\Delta \frac{[\mathbf{E}]_{\mathbf{i}}+[\mathbf{E}]_{\mathbf{i}-\mathbf{1}}}{2}\right.} \\
& \left.+\left([\mathbf{C}]-\frac{\Delta t}{2}[\mathbf{G}]_{i}\right)[\mathbf{V}]_{i-1}\right]
\end{aligned}
$$

where $i$ is the index denoting the current time stamp.

\subsection{Internal Boundary Yee-cell Model}

The developed FDTD formulation of a space-time modulated metasurface determines the scattered fields just before and after a zero-thickness surface for a given time-domain input wave. To better visualize the scattered fields in space and time as well as include the diffraction effects of a finite size metasurface, the FDTD equation of (3.20) can be solved on a conventional Yee-cell grid. Fig. 3.3 shows a numerical setup consisting of a zero-thickness metasurface that is located along the $x$-axis at $z=0$ and with a length of $\ell$ that is much larger than the wavelength of the input signal. For simplicity, a $2 \mathrm{D}$ problem is considered here so that any variations in the fields along the $y$-axis are assumed zero. The $2 \mathrm{D}$ computational domain is surrounded by PMLs to eliminate any backscattering from the boundaries. A hard input source is specified for E-fields at $z=0_{-}$, which in general, is both a function of space and time, to model broadband time-domain pulses and arbitrary spatial wavefronts.

The transmitted fields $E_{t}\left(x, z=0_{+}, t\right)$ that are generated from the metasurface are first determined using (3.20) and subsequently forward-propagated in free-space on a conventional Yee-cell for $z>0$ forming the transmission region. Similarly, the reflected fields $E_{r}(x, z=$ $\left.0_{-}, t\right)$ are backward-propagated for $z<0$, forming the reflection region, as shown in Fig. 3.3. The Yee-cell discritization is determined by the highest expected frequency and the time step set to satisfy the Courant-Friedrichs-Levy criteria [26].

The metasurface parameters are assumed to be the same as that in Fig. 3.1 exhibiting the double-Lorentzian surface susceptibility functions, as obtained in Sec. 3.1. To emulate a space-time modulated metasurface, the resonant frequencies of each Lorentzian contributions 


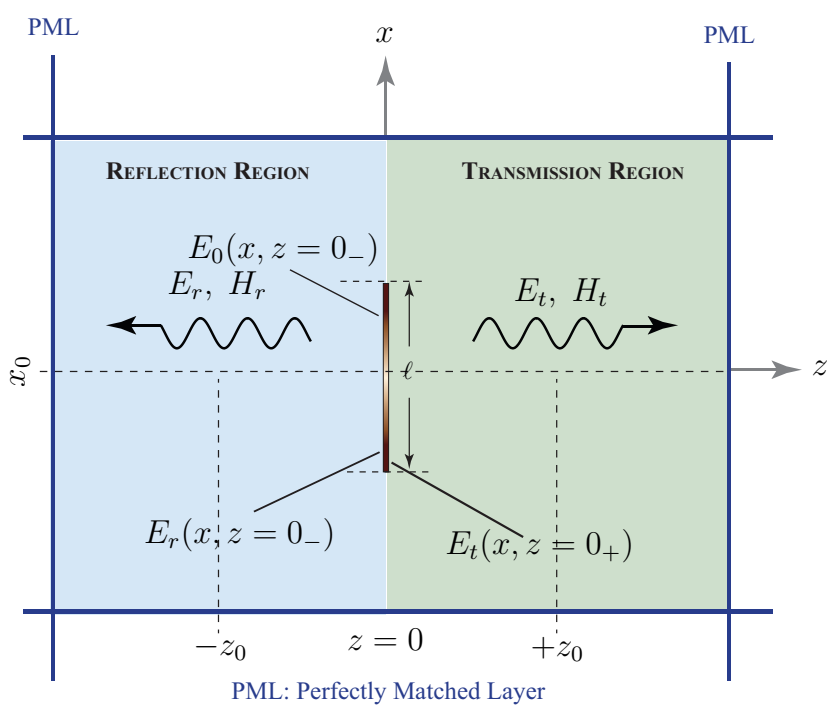

Figure 3.3 Simulation setup showing the numerical domain for a time-domain simulation including a finite-size metasurface of length $\ell$, and the surrounding boundary conditions. Shaded regions on the left and right represent the 2D discretized region where Maxwell's equations are solved on a conventional Yee cell. Figure sourced from [2].

are varied sinusoidally with a pumping frequency of $f_{p}$ and spatial frequency $\beta_{p}$. Finally, the Yee-cell region is discretized along $x$ - and $z$-directions using $n_{x} \times\left(n_{z}=3 n_{x}\right)$ space samples, respectively ${ }^{6}$.

Figure. 3.4 summarizes the key steps of the method in solving a general space-time modulated metasurface. For a given Huygen's metasurface, the space-time modulation is applied to the material permittivity, $\epsilon_{r}(x, t)$, as considered here. To model this surface, a unit cell is chosen (an all-dielectric cell is considered in this thesis) and full-wave simulated with varying permittivities within the range of $\epsilon_{r} \in\left[\min \left\{\epsilon_{r}(x, t)\right\}, \max \left\{\epsilon_{r}(x, t)\right\}\right.$. Using (3.2), their equivalent electric and magnetic susceptibilities, $\tilde{\chi}_{\mathrm{ee}}\left(\epsilon_{r}\right)$ and $\tilde{\chi}_{\mathrm{mm}}\left(\epsilon_{r}\right)$, are extracted and modelled using Lorentzian functions (3.3). The variations in the various parameters of the Lorentzian distributions are then recorded for each permittivity value (ex: Fig. 3.2), mapping the space-time modulation of the permittivity onto the Lorentzian parameters.

Once the pre-processing step is completed, the metasurface can be simulated. At each time instant, various system matrices are evaluated, and the field equation (3.20) is solved for a given input field at each metasurface location. Once the entire reflected and transmitted fields are evaluated at $z=0_{-}$and $z=0_{+}$, respectively, they are used as hard sources in

\footnotetext{
${ }^{6}$ This ensures uniform meshing throughout the simulation domain. In general, a non-uniform meshing can be easily incorporated in the proposed method.
} 


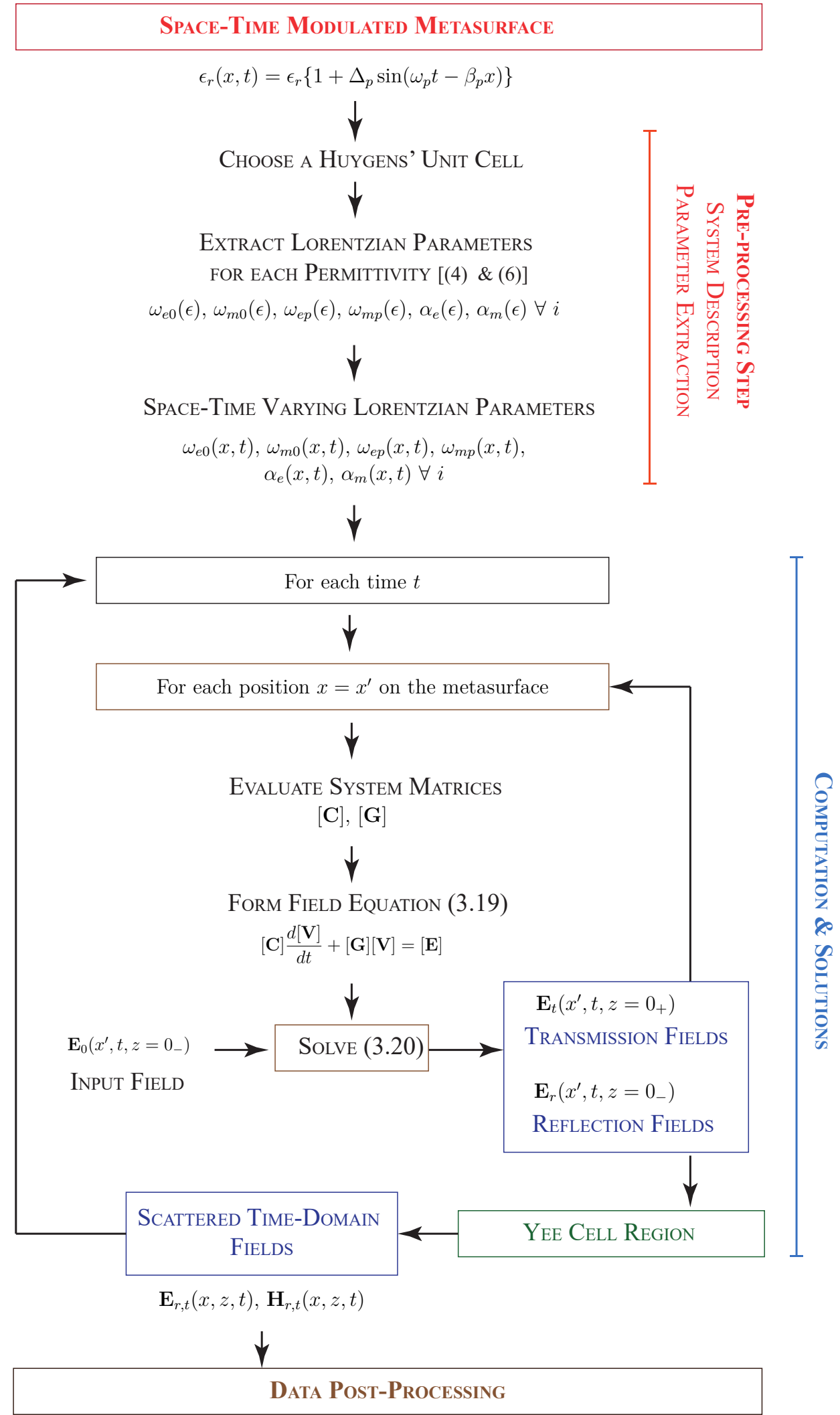

Field Probing, Temporal and Spatial Fourier Transforms

Figure 3.4 Simulation flow chart illustrating the key steps in solving a space-time modulated metasurface using the proposed method. Figure sourced from [2]. 
the Yee-cell region to subsequently compute the scattered fields in the rest of computational domain.

After the simulation is completed the scattered fields at the desired location within the region are post-processed. This involves analyzing the fields in both the spatial and temporal frequency domains to determine the harmonic generation and their angular refraction effects, all of which is described in detail in Section 3.5.

\subsection{Space-Time Modulated Metasurface}

The formulation thus far assumes that the permittivity, and by extention the resonant frequency, is only a function of time. However, to model a space-time modulated metasurface, the spatial modulation $\beta_{p}$ has to be considered. From this, Eq. (3.4) is modified for the more general case of space-time modulation:

$$
\begin{gathered}
\omega_{e 0}(x, t)=\omega_{e 0}+f_{e 0}(x, t)=\omega_{e 0}+\Delta \omega_{e} \sin \left(\omega_{p} t-\beta_{p} x\right) \\
\omega_{m 0}(x, t)=\omega_{m 0}+f_{m 0}(x, t)=\omega_{m 0}+\Delta \omega_{m} \sin \left(\omega_{p} t-\beta_{p} x\right)
\end{gathered}
$$

This periodic space-time perturbation of the resonant frequencies generates several harmonic waves with different frequencies and wave numbers. If the input field is normally incident, the transmitted instantaneous fields just after the metasurface at $z=0_{+}$can in general be expanded into a Floquet series as

$$
E_{t}\left(x, z=0_{+}, t\right)=\sum_{n=-\infty}^{\infty} a_{n} e^{j \omega_{n} t} e^{j \beta_{n} x}
$$

where $a_{n}$ is the coefficient of the Floquet term, $\omega_{n}=\omega_{s}+n \omega_{p}$ is the frequency of the $n^{t h}$ harmonic, and $\beta_{n}=\beta_{x 0}+n \beta_{p}$ is the wavenumber of the $n^{\text {th }}$ harmonic with $\beta_{x 0}=0$ due to normal input incidence. Each harmonic term of this expansion with a temporal frequency $\omega_{n}$ represents an oblique forward propagating plane-wave along the $+z$ direction with an angle $\theta_{n}$ measured from the normal of the metasurface, given $\mathrm{by}^{7}$

$$
\theta\left(\omega_{n}\right)=\sin ^{-1}\left\{\frac{n}{\left(1+n \omega_{p} / \omega_{s}\right)} \frac{\beta_{p}}{k_{0}}\right\},
$$

where $k_{0}$ is the free-space wavenumber of the fundamental frequency. Similarly, the reflected fields can also be expanded in a Fourier series, with different set of harmonic amplitudes $b_{n}$,

\footnotetext{
${ }^{7}$ Also valid for the temporal harmonics of the reflected fields.
} 
which will also be refracted along different angles given by (3.23).

\subsection{Spatial and Temporal Fourier Transform Analysis}

In its most general form, a space-time modulated metasurface will produce several harmonic frequencies that propagate in different directions, as described in Eq. (3.22). When viewing the propagated field in the Yee-cell it is difficult to determine which of the wavefronts corresponds to what harmonic, especially closer to the metasurface where all of the harmonics overlap with each other. In order to compare the various profiles of the fields at a specific point along their propagation a new technique needed to be developed.

For a given simulation the transmitted field $E_{t}$ is recorded along $x$ at a distance of $+z_{0}$ away from the metasurface, as shown in Fig. 3.3. The time-domain signal can't provide too much meaningful information by itself, and thus it has to be processed. After the simulation is finished a temporal Fourier transform is performed using an FFT at each point along $x$ on the steady-state portion of the signal (for consistency, this is usually performed on the second half of the simulation). This provides the electric field in the frequency domain $\tilde{\mathbf{E}}\left(x, z=z_{0}, \omega\right)$ as a function of $x$ and $\omega$.

For each of the analyzed harmonics the frequency domain field is sampled at the required frequency $\omega_{n}$. This provides a spatial profile of the field for that particular frequency along the $x$ direction. In general the field is a complex number, and if all that is wanted is the overall shape of the field then the absolute value can be taken. Therefore the spatial profile of the $n^{\text {th }}$ harmonic at $z=z_{0}$ can be described by:

$$
\tilde{\mathbf{E}}_{n}\left(x, z=z_{0}\right)=\left|\tilde{\mathbf{E}}\left(x, z=z_{0}, \omega_{n}\right)\right|
$$

Although this is done in the frequency domain it offers an accurate view for what the field is doing once it has reached a steady state. Although this profile can be used in its current form to find the angle of refraction of the harmonics there is a better method that has been discussed already in Sec. 2.2.2. Because the frequency-domain signal is a complex number it contains the relative phase information of the field along $x$. Therefore, if a spatial Fourier transform is performed on the spatial profile of the field (before the absolute value is taken) the strengths of the spatial frequencies of the $n^{t h}$ harmonic $k_{n, x}$ can be obtained. This leads to:

$$
\tilde{\mathbf{E}}_{n}\left(k_{x}, z=z_{0}\right)=\left|\mathcal{F}_{x}\left\{\tilde{\mathbf{E}}\left(x, z=z_{0}, \omega_{n}\right)\right\}\right|
$$


Eq. (2.27) gives the formula for calculating the angle of refraction for a single frequency. When the different harmonics are taken into account, it transforms into:

$$
\theta_{n}=\sin ^{-1}\left(\frac{k_{n, x, \max }}{k_{n}}\right)
$$

where $\theta_{n}$ is the angle of refraction measured from the normal, $k_{n}=\omega_{n} / c$ is the wavenumber of the given frequency in free-space, and $k_{n, x, \max }$ is the spatial frequency corresponding to the peak value of $\tilde{\mathbf{E}}_{n}\left(k_{x}, z=z_{0}\right)$. 


\section{Chapter 4 Results}

In order to properly demonstrate the various modelling and analysis techniques that were outlined in Chapter 3 various examples are presented. To provide an accurate comparison of the different methods, as well as the effects of the various modulation schemes, the same metasurface needs to be simulated for each case.

The metasurface is assumed to be built using unit cell \#2 from Fig. 3.1 with its equivalent Lorentzian parameters which are described in the figure. Unless otherwise stated, the permittivity of the metasurface is held at $\epsilon_{r}=11.9$, and the normally incident wave has a frequency of $\omega_{0}=2 \pi(230 \mathrm{THz})$.

\subsection{Single Point Simulations}

When a Hyugens' metasurface is excited by a normally incident wave each point along the metasurface can be treated as a point source for the transmitted and reflected regions. Because of this behaviour, it is both possible and advantageous to model a single point on the metasurface. Collapsing the simulation to a single point solution provides a good insight into the shape of the output waveforms under different metasurface excitations without the effects of dispersion.

\subsubsection{Static Surface}

To demonstrate this method let us first consider a linear time-invariant (LTI) metasurface. Fig. 4.1 shows the transmitted and reflected fields of the metasurface due to a monochromatic wave normal to the surface in the time domain. The incident wave $E_{0}(t)$ is slowly increased to its full value over the course of 200 fs using a Gaussian curve with a time variance of $\sigma_{t}=100$ fs. This produces an incident wave that is initially broadband but collapses into a single frequency.

This simulation provides a bit of insight into the response of the metasurface. At the beginning of the simulation, the transmitted field increases and then narrows near $t=175 \mathrm{fs}$, only to increase once again towards its steady-state value. Initially the transmitted field would be in phase with the incident field and over time its phase needs to shift towards its steady state value. During this transition the magnitude of the transmitted field distorts. The reflected field has a similar distortion at the beginning, albeit at a later time than the transmitted field. The reflected field increases past its steady state value, slowly decreasing 


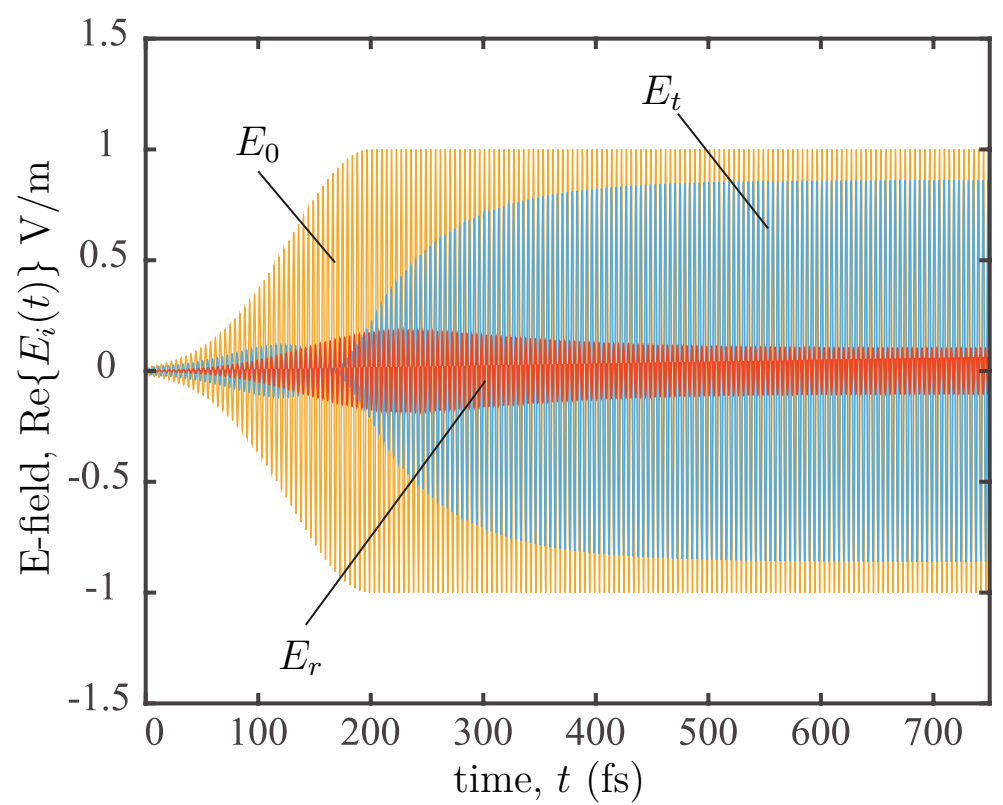

Figure 4.1 Response of a linear time-invariant metasurface, showing the instantaneous E-field of the time-domain reflected and transmitted fields at the metasurface.

again until it becomes steady again.

\subsubsection{Time-Varying Surface}

A simple extension to the single point simulation is to model a time modulated metasurface. Fig. 4.2 shows the transmitted and reflected fields of a metasurface that has a small modulation frequency of $\omega_{m}=0.025 \omega_{0}$ and a modulation index of $\Delta_{p}=0.1$. The incident wave is the same wave that was used in the static metasurface example to provide a good comparison between the different waveforms.

It can be seen from these waveforms that both the transmitted and the reflected fields produce temporal profiles that are similar to a beat frequency. This effect is caused by the generation of new harmonics inside of the field, which is produced by the time modulation of the metasurface. In order to gain a better understanding of the signal's harmonic composition an FFT is done on the transmitted and reflected fields once the metasurface has reached a steady state.

Fig. 4.3 shows the power of the Fourier transforms of (a) the transmitted and (b) the reflected fields. In both of the profiles it can be seen that the harmonics align with what was discussed in Sec. 3.4, where the fundamental frequency remains at $\omega_{0}=2 \pi f_{0}$ and the harmonic frequencies are equally spaced following $\omega_{n}=\omega_{0}+n \omega_{m}$ for the $n^{\text {th }}$ harmonic. 


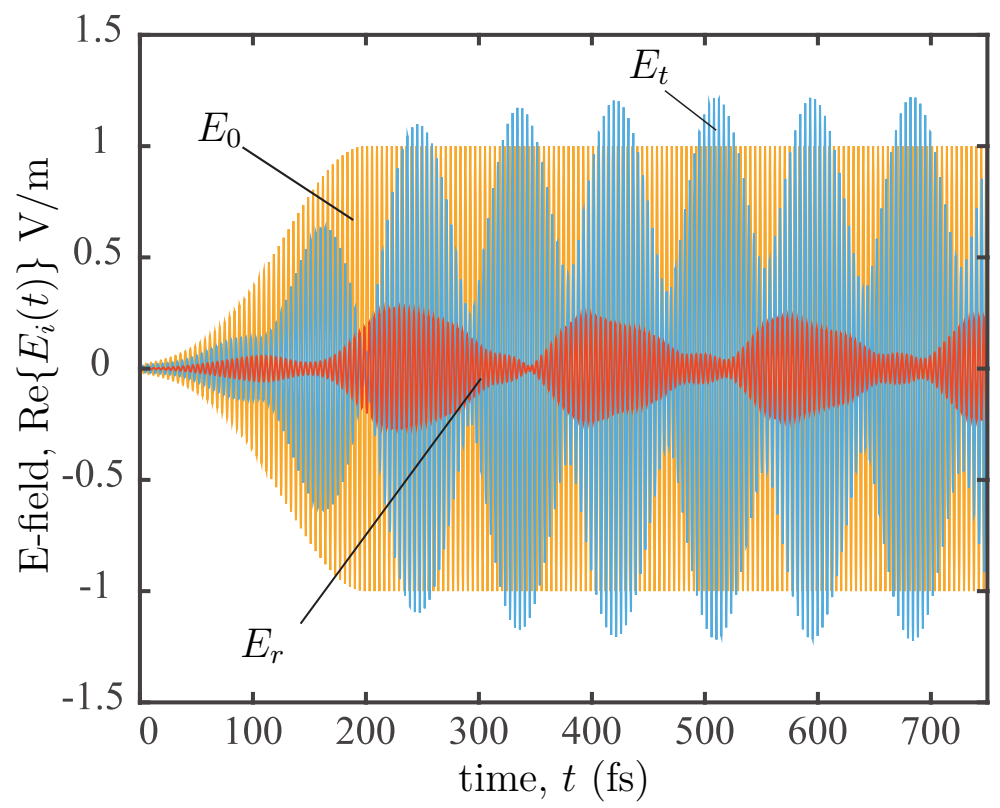

Figure 4.2 Response of a time modulated metasurface with $\omega_{m}=0.025 \omega_{0}=2 \pi(5.75 \mathrm{THz})$ and $\Delta_{p}=0.1$, showing the instantaneous E-field of the time-domain reflected and transmitted fields at the metasurface.

An interesting phenomenon that can be seen from this simulation is that the fundamental harmonic of the transmitted field is considerably lower than the neighbouring harmonics, especially considering that this is not observed in the reflected field's profile. This is discussed in more detail in the following sections where the 2D simulations can provide a better visual aid.

\subsection{D Simulations}

Because we are now dealing with a 2D region the size of the metasurface as well as the shape of the incoming electromagnetic field have to be properly defined. Unless it is otherwise specified, the simulation setup follows the setup that is defined in Sec. 3.3. To ensure that the metasurface is electrically large a surface of length $\ell=25 \mu \mathrm{m}$ is placed in the middle of a region of dimensions $x=[0,50] \mu \mathrm{m}, z=[0,150] \mu \mathrm{m}$.

The spatial profile of the electric field either follows a Gaussian distribution or a plane wave distribution. For plane waves, it is assumed that the field across the entire wavefront is equal with a magnitude of one and a frequency of $\omega_{0}=2 \pi(230 \mathrm{THz})$. The Gaussian beam is set to have a max value of one, a standard variation of $\sigma_{x}=5 \mu \mathrm{m}$, a median of $x_{\text {avg }}=25 \mu \mathrm{m}$, and a frequency of $\omega_{0}=2 \pi(230 \mathrm{THz})$. 


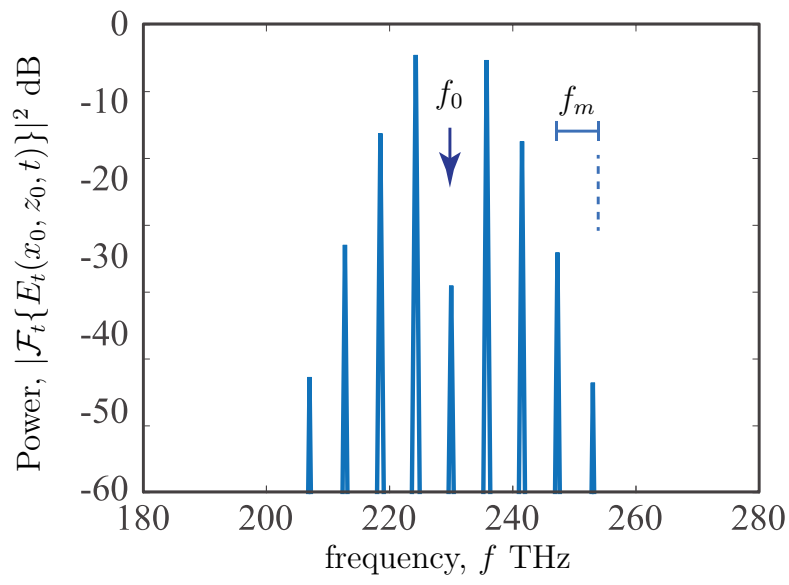

(a)

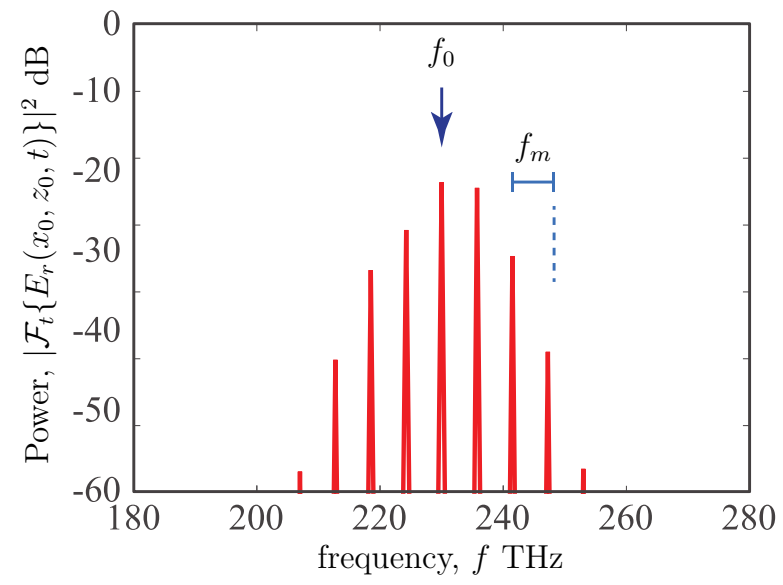

(b)

Figure 4.3 Temporal Fourier transform of the output fields from the time modulated metasurface with $\omega_{m}=0.025 \omega_{0}=2 \pi(5.75 \mathrm{THz})$ and $\Delta_{p}=0.1$ once it has reached steady state. This shows (a) the power of the transmitted field as a function of frequency and (b) the power of the reflected field as a function of frequency, both expressed in $\mathrm{dB}$.

\subsubsection{Fourier Propagation Examples}

\section{Single Frequency Static Surfaces}

For our first 2D example let us consider again the case of a static metasurface. The steps to solve this case are outlined in Sec. 2.2.2. A continuous wave (CW) Gaussian beam is incident on the surface and the steady-state transmitted field is extracted and then propagated using the Fourier propagation techniques. The spatial resolution along the surface is set to be $\Delta_{x}=125 \mathrm{~nm}$ in order to provide an accurate picture of the field.

Fig. 4.4 shows the propagated field through a region of dimensions $x=[0,50] \mu \mathrm{m}$, $z=[0,50] \mu \mathrm{m}$ where the metasurface is on the left-hand side of the region. The field dissipates in the region which can be seen from the broader profile on the right end of the simulation region than the left end and with a lower intensity, which is expected. While this does show that the Fourier propagation techniques do properly model the dissipation and the evolution of the field profile from the steady-state source it can be expanded upon in the more general case.

\section{Harmonic Generation and Propagation}

Let us consider the same metasurface with a space-time modulation of its permittivity that follows Eq. (3.1) with modulation parameters of $\Delta_{p}=0.1, \omega_{m}=0.025 \omega_{0}=2 \pi(5.75 \mathrm{THz})$, 


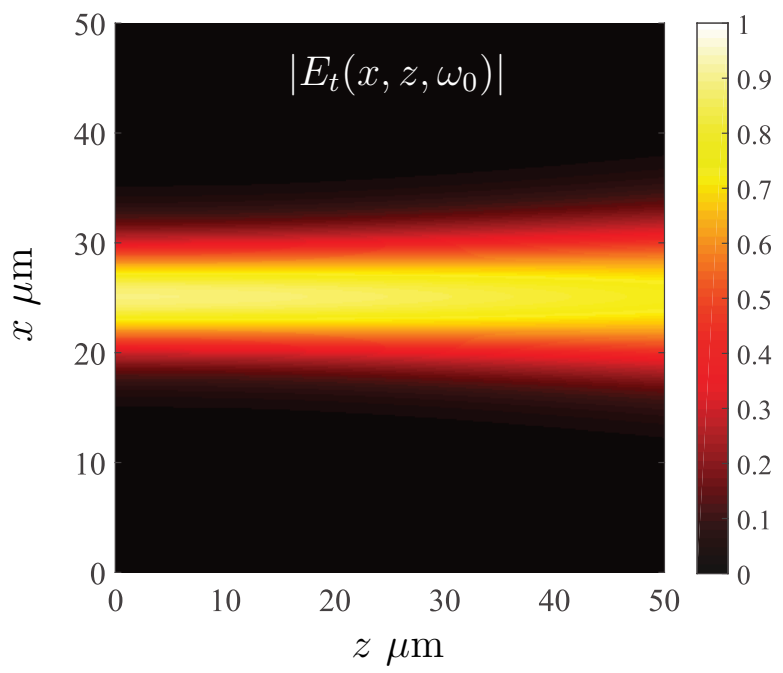

Figure 4.4 Fourier propagated fields in the transmission region for the static metasurface. The maximum field is $\max \left\{\left|E_{t}(x, z, \omega)\right|\right\}=0.8615$.

and $\beta_{m}=4 \pi \times 10^{5} \mathrm{rad} / \mathrm{m}$. A monochromatic Gaussian beam with a unit amplitude is incident on the metasurface at $z=0_{-}$and the transmitted field is captured and processed using the formulation outlined in Sec. 4.4, keeping in mind that due to the modulation several different frequencies will be generated.

Fig. 4.5(a) shows the spatial spectrum of the transmitted waves at the output of the metasurface for each dominant harmonic. Due to the space modulation of the surface, each harmonic component exhibits a distinct spectral peak in $k_{x}$. The locations of these peaks can be used with Eq. (2.27) to calculate the angles of refraction from the normal. Fig. 4.5(b) compares these calculated angles with the theoretical angles determined using Eq. (3.23). From this figure, it can be seen that the calculated angles match well with the analytical solution.

To further compute the propagation characteristics of the metasurface output the first few harmonics of the transmitted field are propagated. Fig. 4.6 shows the Fourier propagation of the harmonics corresponding to $n= \pm 1$. The fundamental mode, $n=0$, was not shown because it's peak field strength was significantly smaller than its neighbouring harmonics, with $\max \left\{\left|E_{t}\left(x, z, \omega_{0}\right)\right|\right\}=0.0211$. The spatial Fourier transform from Fig. 4.5 shows that the fundamental frequency is very low in comparison to the neighbouring harmonics, and this is mirrored in the Fourier propagated fields.

The two harmonics shown diverge from the normal of the metasurface and have similar maximum values for this modulation. Similarly to the static case that is studied in Sec. 4.2.1, 


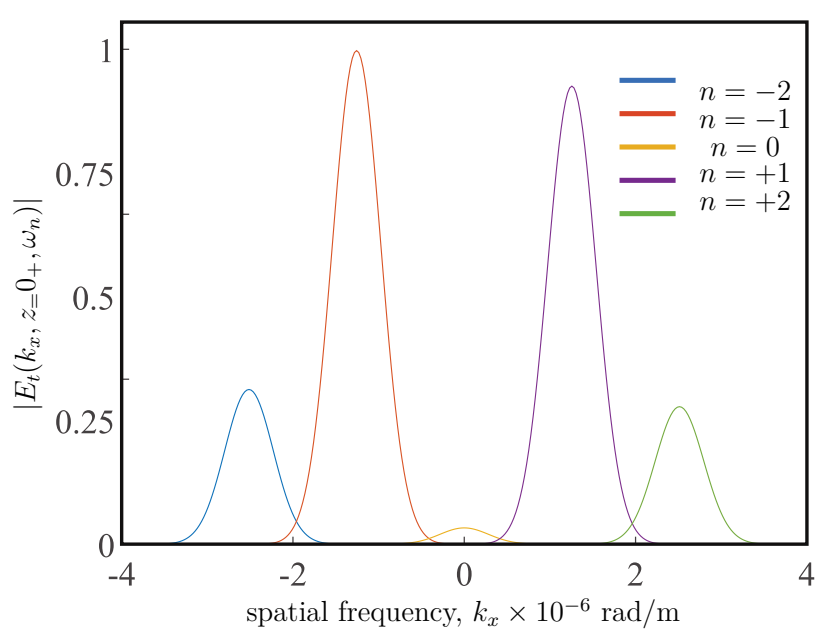

(a)

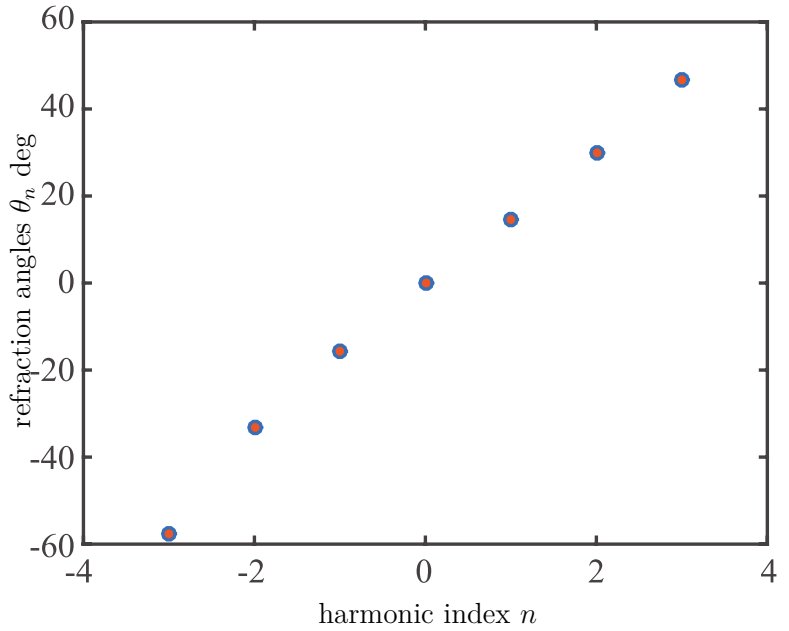

(b)

Figure 4.5 Output field characteristics of a matched space-time modulated metasurface excited with an normally incident Gaussian beam. (a) Spatial Fourier transform at the output of the metasurface at $z=0_{+}$for first few harmonics, normalized to the max value of the largest generated harmonic. (b) The refraction angle of each harmonic using (3.26). Metasurface parameters: $25 \mu \mathrm{m}$ in size, and mesh size $\Delta x$ of $125 \mathrm{~nm}$. The Gaussian beam parameters: $\omega_{0}=2 \pi(230 \mathrm{THz})$, and standard variation of $\sigma_{x}=5 \mu \mathrm{m}$. Modulation parameters: $\Delta_{m}=0.1$, $\beta_{m}=4 \pi \times 10^{5} \mathrm{rad} / \mathrm{m}$ and $\omega_{m}=0.025 \omega=2 \pi(5.75 \mathrm{THz})$. Figure sourced from [3].
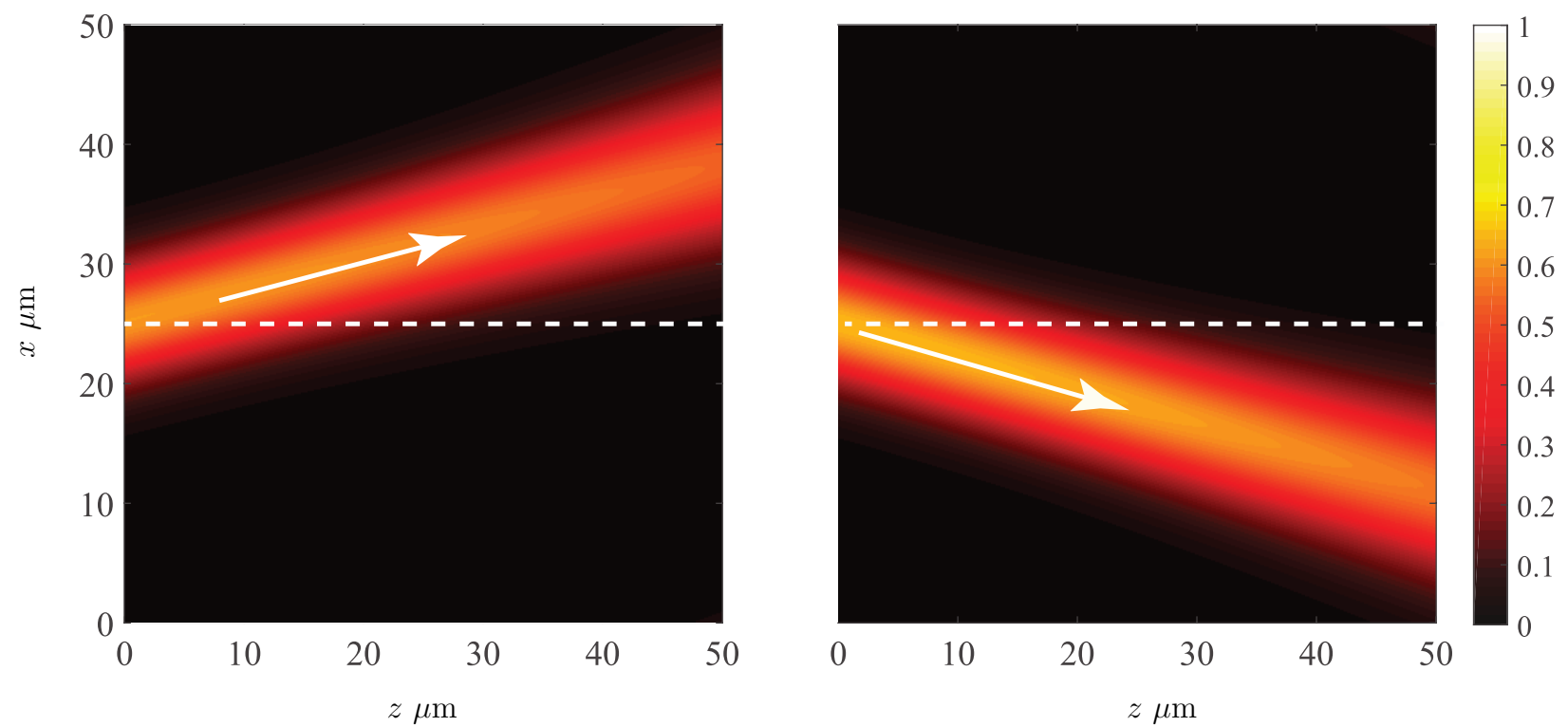

Figure 4.6 Fourier propagated fields in the transmission region of the metasurface of Fig. 4.5 showing the first up- and down-converted harmonics. $\max \left\{\left|E_{t}\left(x, z, \omega_{+1}\right)\right|\right\}=0.5942$, and $\max \left\{\left|E_{t}\left(x, z, \omega_{-1}\right)\right|\right\}=0.6403$ 
the waveforms experience dispersion as they propagate from the metasurface on the left end of the simulation region to the right. This example demonstrates that the Fourier propagation techniques properly model the steady-state fields through space for a source that has broadband harmonic content.

\subsubsection{Yee Cell}

\section{Static Metasurface}

Let us consider a static metasurface first. The metasurface is assumed to be built using unit cell \#2 of Fig. 3.1 with its equivalent Lorentzian parameters and is solved using the method outlined in Chapter 3. Fig. 4.7(a) shows the scattered fields when a continuous wave with a plane-wavefront is incident normally on the metasurface. Since unit cell \#2 exhibits a finite reflection (mismatched due to unequal $\chi_{\text {ee }}$ and $\chi_{\mathrm{mm}}$ ), fields are manifest in both the transmission and reflection regions. Furthermore, since a plane-wave is assumed, the $x$ component of the H-field is negligible throughout, except at the two extremities of the metasurface capturing the fringing fields. The CW wave is switched on with a slowly rising Gaussian edge, and the corresponding time-domain fields at the centre of the reflection and transmission regions, at $\left(x_{0},+z_{0}\right)$ and $\left(x_{0},-z_{0}\right)$ respectively, are shown in the middle of Fig. 4.7(a). The signal frequency $f_{s}=230 \mathrm{THz}$ is close to the first Lorentzian resonant frequency, which leads to a strong interaction of the input wave with the metasurface. This creates a distorted transition before the reflected and transmitted signals reach a steady state.

To validate these results, the computed fields are compared with theory in the spectral $\left(k_{x}\right)$ domain. This is achieved by computing $\tilde{E}_{y}\left(k_{x}, z_{0}, \omega_{0}\right)$ using the technique described in Sec. 3.5 and comparing it with the spatial Fourier transform of the input. This comparison is shown on the right of $4.7(\mathrm{a})$, where the theoretical distribution corresponds to the spatial Fourier transform of an ideal aperture (of size $\ell$ ) with constant field distribution. A good agreement is seen between the two. A smaller amplitude is seen for the computed spectrum, which can be attributed to dissipations losses of the metasurface and the energy being absorbed in the PMLs. The same procedure is repeated for a Gaussian beam and a step transition to CW as shown in 4.7(b), where a Gaussian beam diffracts through free-space after hitting the metasurface, again showing a good agreement with theoretical predictions.

Finally, a normally incident Gaussian pulse with a standard variation of $\sigma_{t}=50$ fs is simulated on the the uniform metasurface of Fig. 4.7 in order to test how well the model handles broadband input signals. Fig. 4.8(a) shows the Gaussian wavefront at two different 
(a)
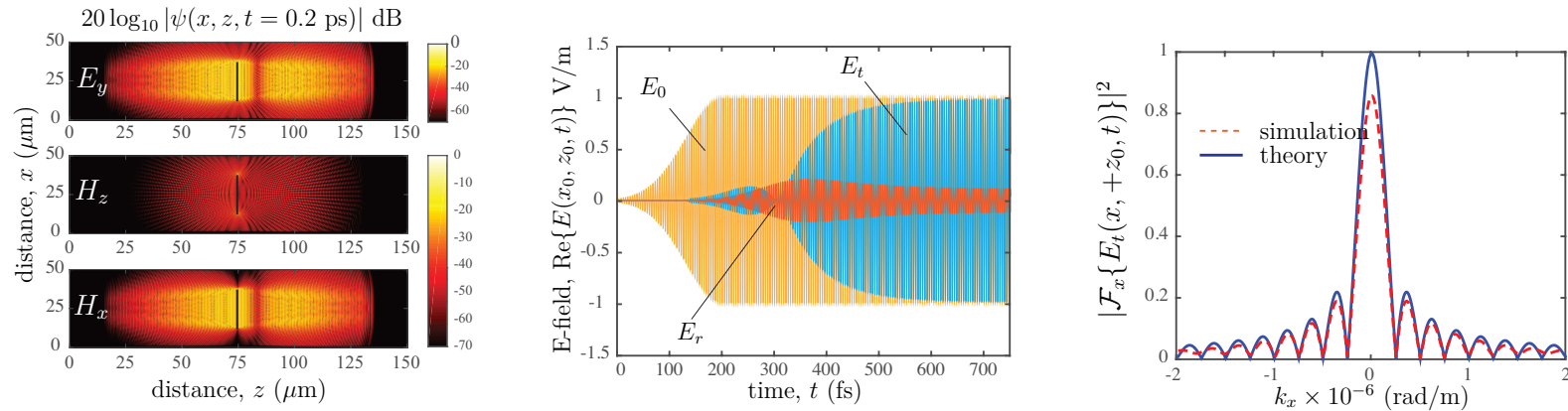

(b)
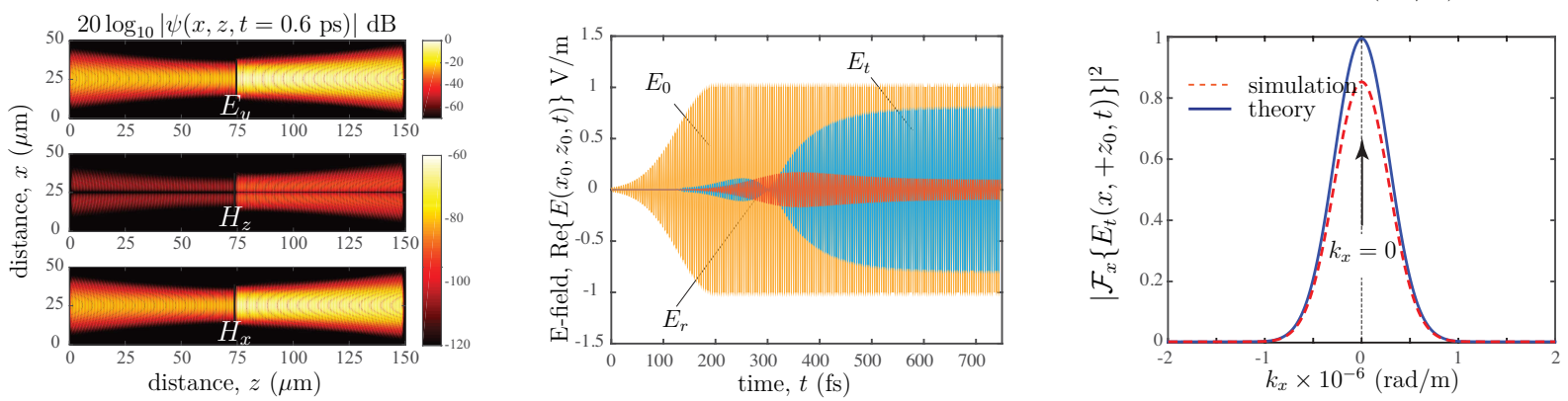

Figure 4.7 Response of a linear time-invariant uniform metasurface, showing the instantaneous E-field and H-field distributions, corresponding time-domain reflected and transmitted fields at the field-probing locations $\left(x_{0}, \pm z_{0}\right)$, and the spatial Fourier transform of the fields in a transverse cross-section at $z=z_{0}$, for the two cases of (a) normally incident plane-wave, and (b) normally incident Gaussian-beam. The simulation parameters are as follows: CW frequency $f_{s}=230 \mathrm{THz}, \ell=25 \mu \mathrm{m}$, Gaussian field at the input $E_{y}\left(x, z=0_{-}\right)=\exp \left[-\left(x-x^{\prime}\right)^{2} / \sigma_{x}^{2}\right]$, with $x^{\prime}=25 \mu \mathrm{m}, \sigma_{x}=5 \mu \mathrm{m}$. The rising Gaussian edge for plane-wave case is $\sigma_{t}=100 \mathrm{fs}$. Meshing $n_{x}=400$. The metasurface parameters for are extracted from the unit cell \#2 of Fig. 3.1. Figure sourced from [2]. 


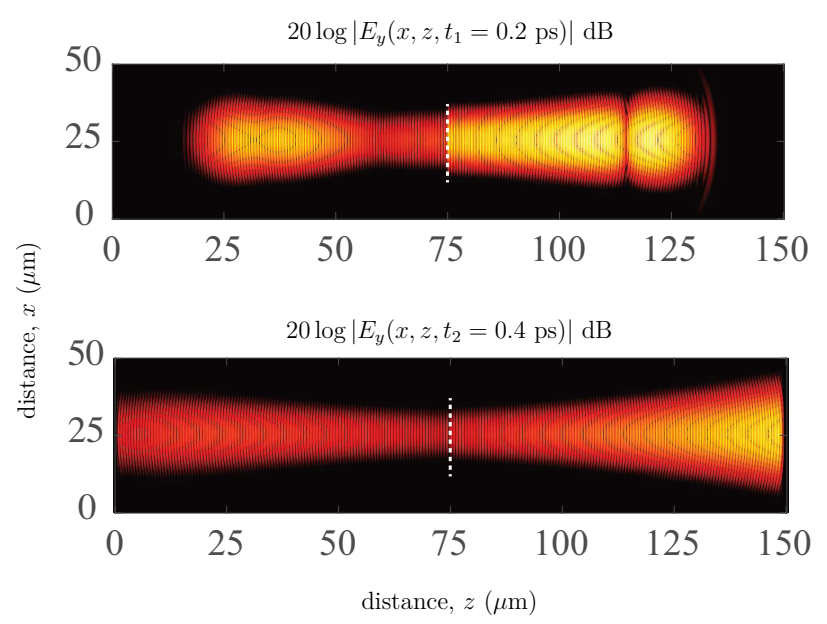

(a)

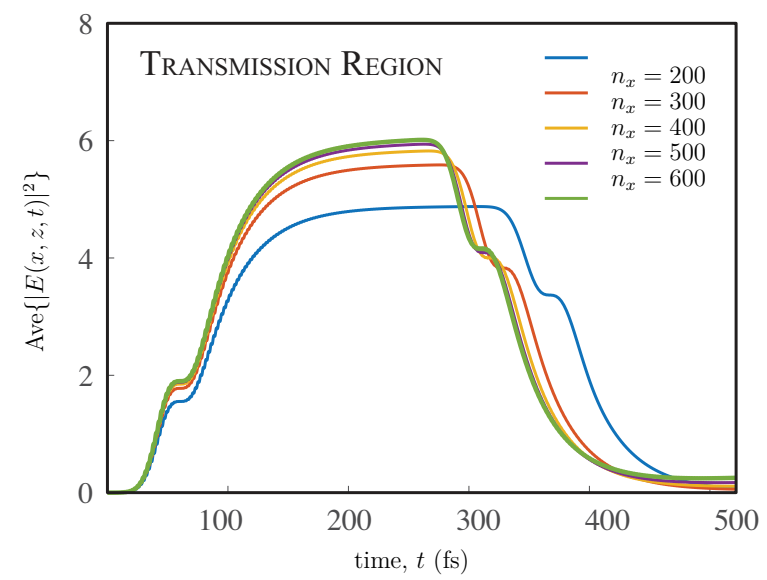

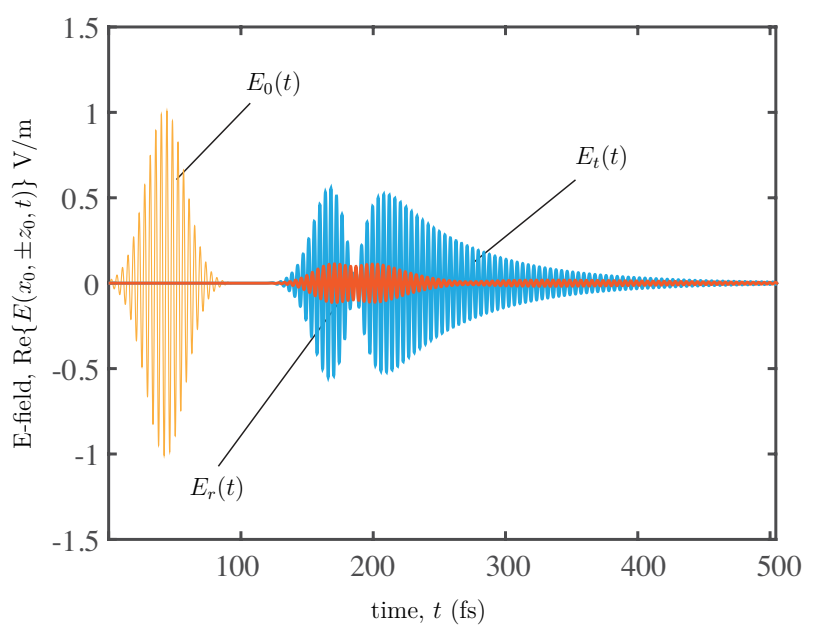

(b)

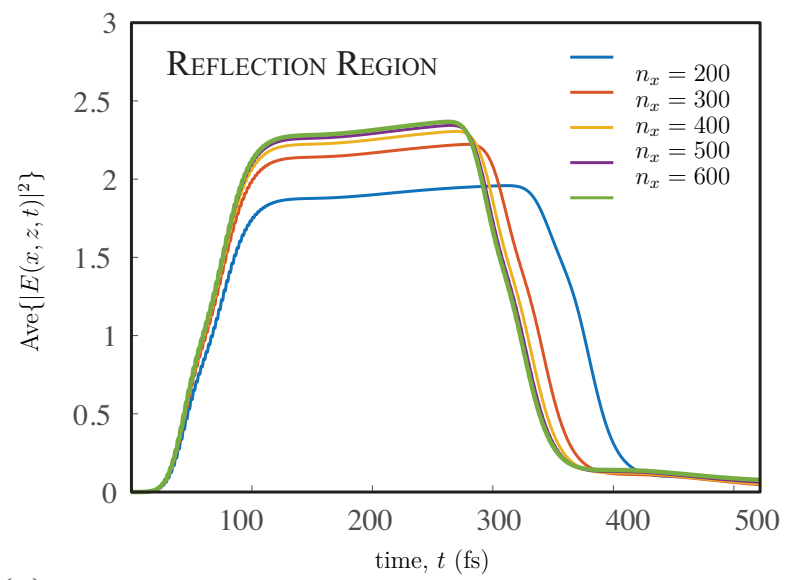

(c)

Figure 4.8 Transmitted and reflection fields from a linear time-invariant metasurface for the case of a pulsed (broadband) Gaussian beam. (a) Spatial E-fields in the entire simulation region at two time instants $t_{1}=0.2 \mathrm{ps}$, and $t_{2}=0.4 \mathrm{ps}$. (b) Time-domain incident, reflected and transmitted fields at the probe locations in the transmission and reflection regions $\left[\left(x_{0},-z_{0}\right)\right.$ and $\left(x_{0},+z_{0}\right)$ ], respectively. (c) The convergence plot of the simulation, where the average energy in the transmission and reflection regions converges at each time instant, for increasing value of the mesh density (related to $n_{x}$, where $n_{z}=3 n_{x}$ ). The gaussian pulse width parameter is $\sigma_{t}=50$ fs. Figure sourced from [2]. 
time instants in order to show how the pulsed wave evolves over time before it exits the simulation region. The corresponding time domain fields are shown in Fig. 4.8(b) for both the reflected and transmitted fields. A strong temporal dispersion with a slowly decaying pulse tail is seen in both of the fields, which is due to the proximity of the carrier frequency $\omega_{0}$ of the Gaussian pulse to the first resonant frequency of the metasurface.

Fig. 4.8(c) shows the convergence of the simulation results, where the mesh grid in the Yee-cell region is continuously adapted by increasing $n_{x}$ until the total energy in the simulation region is converged to a stable value. It is found that $n_{x}>500$ leads to a stable field solution, at each time instant. For all simulations throughout this section, including that of Fig. 4.7, the simulation convergence based on the total average energy in the simulation domain, is thoroughly checked and ensured.

\section{Time Modulated Metasurface}

Next, consider a time-modulated metasurface. Such a metasurface can be achieved by sinusoidally varying the permittivity of the all-dielectric unit cell, as described in Sec. 3.1, using (3.1) with $\beta_{p}=0$, and $\Delta_{p}$ controlling the peak variation in the permittivity.

For this example a normally incident pulsed Gaussian wave is applied to the metasurface in order to test the model's ability to handle broadband input fields when under temporal modulation. The pulse has a center frequency of $\omega_{0}=230 \mathrm{THz}$ with a temporal width parameter of $\sigma_{t}=600 \mathrm{fs}$. The pumping frequency is set to be $\omega_{m}=0.025 \omega_{0}$ (2.5\% of the excitation frequency) with variation in the permittivity of $\Delta_{p}=0.1$, which emulates the general case of a strong reflection from the metasurface. Fig. 4.9(a) shows the scattered fields in the transmission and reflection regions while the pulse is travelling through space. The strong temporal beating indicates that new frequency components have been generated, which is expected from a time-modulated metasurface. This temporal beating is even more evident in the time-domain plot, which shows the transmitted and reflected fields at the recording points along with the incident waveform.

A temporal Fourier transform is performed on the transmitted and reflected fields around the pulses. Fig. 4.9(b) shows these temporal Fourier transforms normalized with respect to the incident field. This shows that the newly generated spectral components are all equally spaced, and the center frequencies follow $\omega_{n}=\omega_{0} \pm n \omega_{m}$ with different peak harmonic strengths. It is also observed that the power in each of the peaks is not concentrated at a specific frequency but is instead distributed in a small range around $\omega_{n}$. This indicates that each of the harmonics that are generated from the broadband Gaussian pulse are also broadband in nature, and each of them have a similar pulse width. 

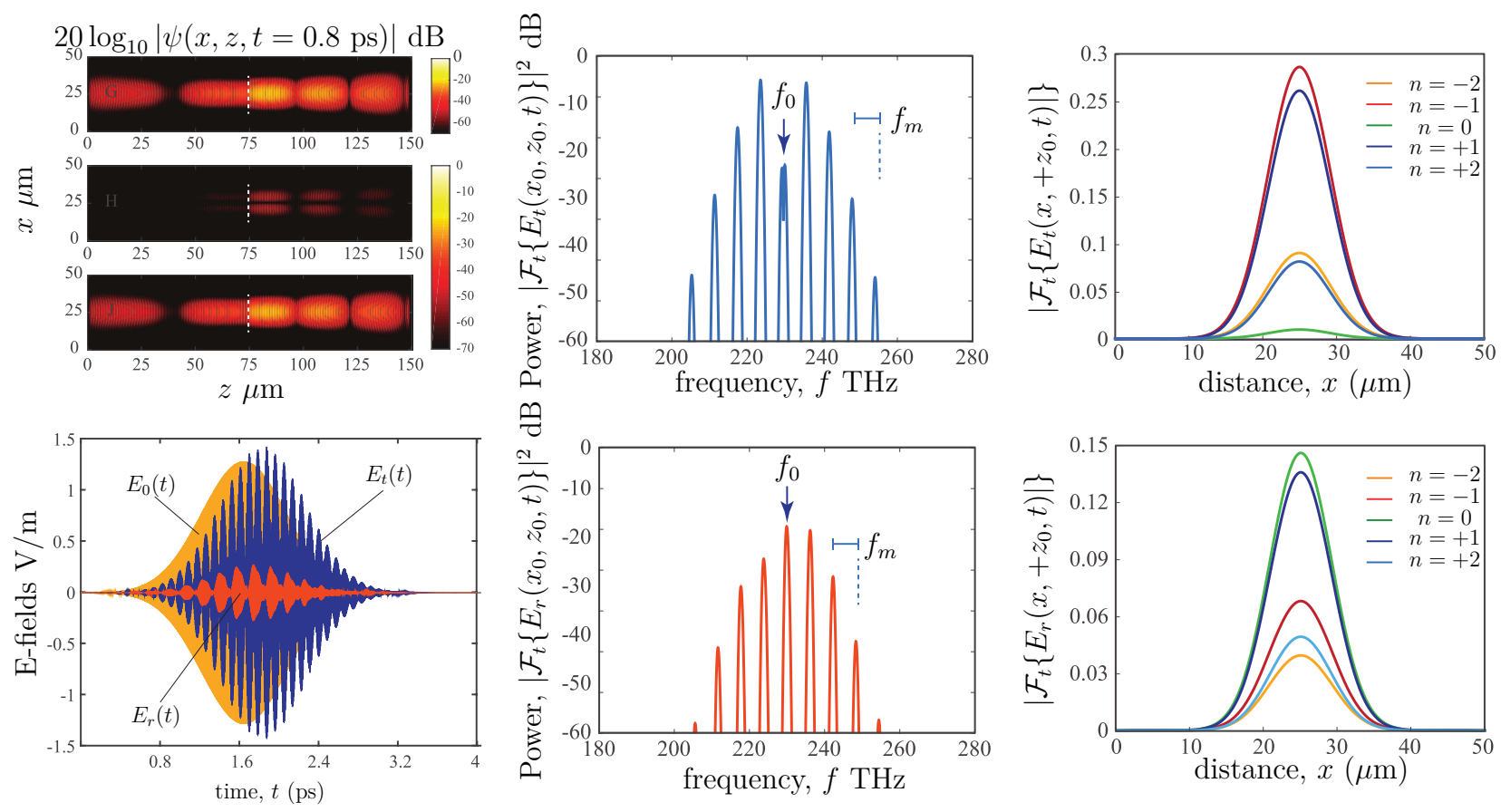

(a)

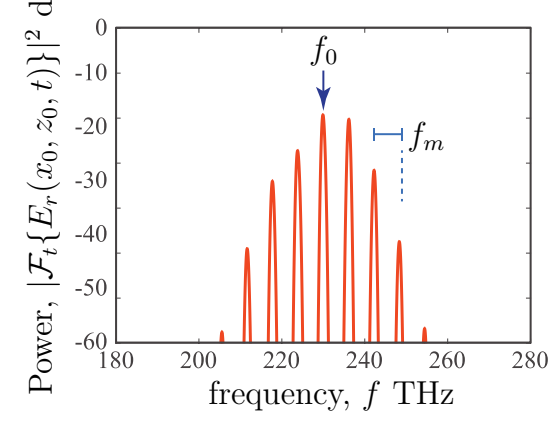

(b)

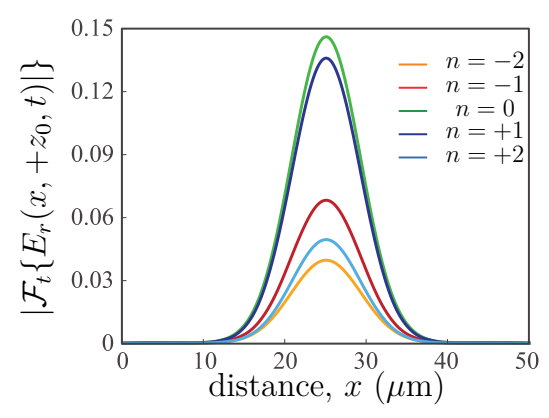

(c)

Figure 4.9 Response of a time-only modulated (uniform) metasurface for a normally incident Gaussian pulsed Gaussian beam with pumping frequency $f_{m}=0.025 f_{0}=5.75 \mathrm{THz}$ and $\Delta_{p}=0.1$, showing a) the time-domain E-fields at $\left(x_{0}, \pm z_{0}\right)$ and its spatial distribution in the transmission and reflection regions at a given time instant, (b) transmission and reflection temporal spectrum at $\left(x_{0}, \pm z_{0}\right)$, and (c) E-field distribution in the transverse plane at $z= \pm z_{0}$, corresponding to transmission and reflection fields, respectively. Only first few spectral components are shown, for clarity. $\mathcal{F}_{t}\{\cdot\}$ and $\mathcal{F}_{x}\{\cdot\}$ denote temporal and spatial Fourier transforms, respectively. The gaussian pulse width parameter is $\sigma_{t}=600 \mathrm{fs}$. Figure sourced from $[2]$. 
A strong frequency conversion is observed where the energy from the fundamental frequency $\omega_{0}$ is transferred equally to both up- and down-converted components in the transmission region. This phenomenon does not always occur, however, and was instead engineered by tweaking the pumping frequency. Interestingly, the relative strengths of these new spectral components are different in the reflection region as compared to that in transmission region. This is expected, however, because the metasurface's transmission and reflection responses are different for each of the spectral component frequencies.

Further, the spatial profiles of the first few harmonics at $z= \pm z_{0}$ are shown in Fig. 4.9(c). All of the newly generated harmonics are aligned spatially, confirming that all the harmonics propagate together along the same direction $\left(\theta_{n}=0^{\circ}\right)$, which is expected from Eq. (3.23).

\section{Space-Time Modulated Metasurface}

For our final example consider a more general case of space-time modulated metasurfaces. The surface's material permittivity, $\epsilon_{r}$, is varied in both space and time following (3.1). Whereas a simple time modulated metasurface generates harmonics that all travel in the same direction, the space-time modulation will produce wavefronts with oblique propagation following (3.23).

Figure. 4.10 shows a demonstration of a space-time modulated metasurface with a pumping frequency $\omega_{m}=0.025 \omega_{0}$ and a spatial modulation of $\beta_{m}=10 \pi / \ell=$. Figure. 4.10(a) shows the transmitted and reflected fields at two different time instants in $\mathrm{dB}$ scale to provide a good contrast between the waveforms. Early on in the simulation at $t=0.2 \mathrm{ps}$ we can see the various wavefronts developing and diverging with different angles. One interesting thing to note from this is that the fundamental frequency mode (the wavefront that is traveling normal to the surface) initially is very strong in the transmission region, appearing to have a strength that is comparable to the neighbouring harmonics. However, once the field has reached a steady state at $t=0.6 \mathrm{ps}$, the fundamental harmonic has significantly dropped off in intensity. This behaviour cannot be seen from the Fourier propagated simulations in Sec. 4.2.1 but could be seen in the time-domain simulation, which demonstrates one of the benefits of the time-domain method.

Once the simulation has reached its steady state the time-domain data can be analyzed using the methods outlined in Sec. 3.5. Figure. 4.10(b) shows the E-field distribution in the transmission and reflection region at $z= \pm z_{0}$ where various harmonics are seen to be clearly separated in space ${ }^{1}$ It should be noted that the fundamental frequency $\omega_{0}$ undergoes

\footnotetext{
${ }^{1}$ The field distribution for $n= \pm 2$ harmonics are not fully seen as they are refracted into the PMLs and
} 

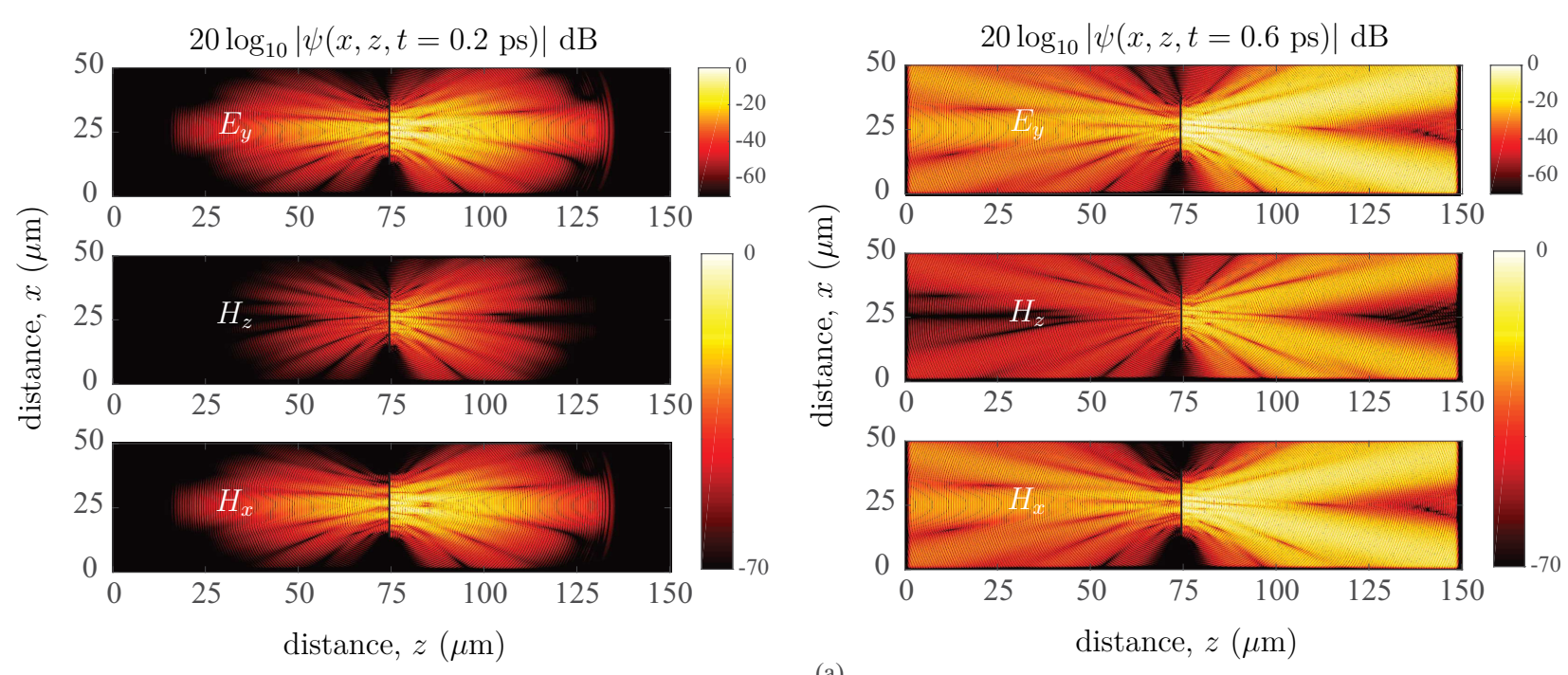

(a)
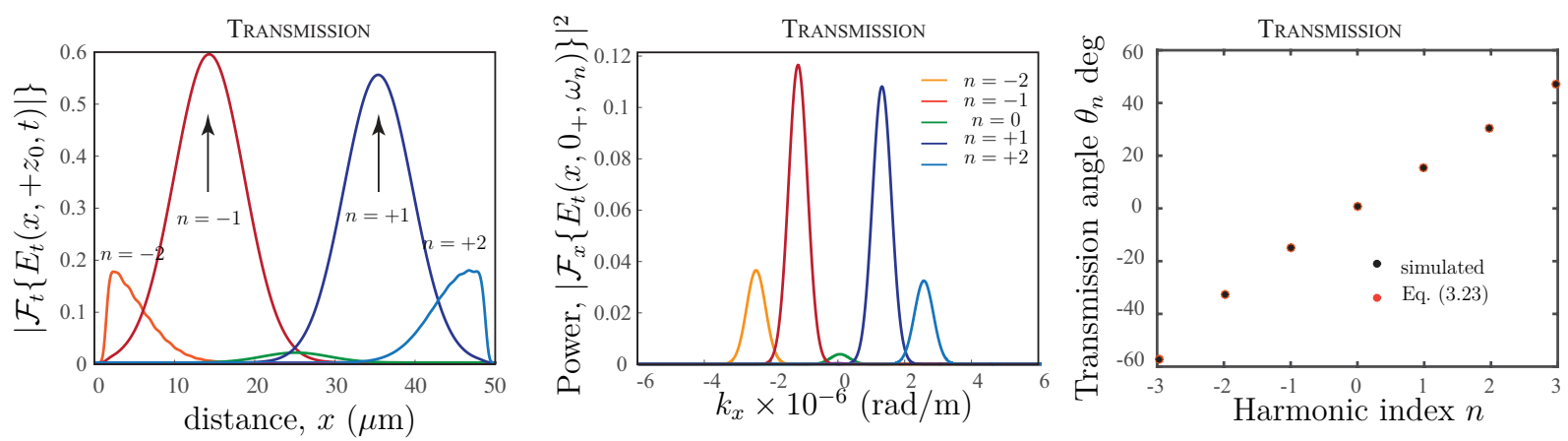

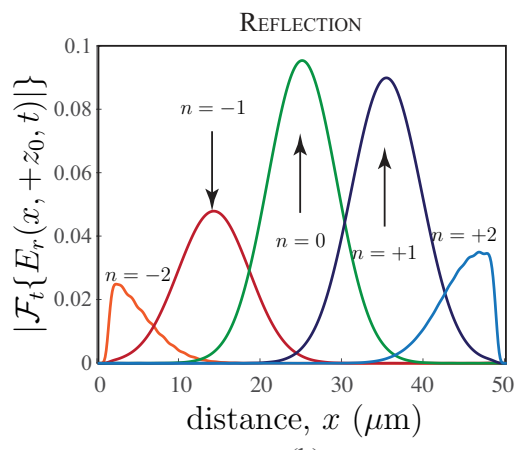

(b)

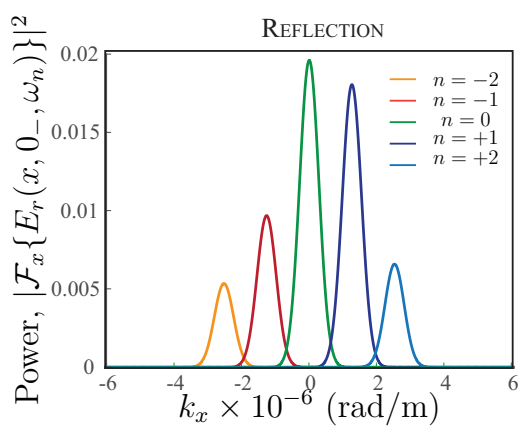

(c)

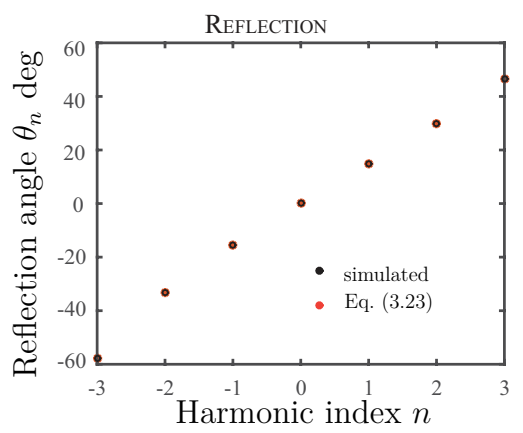

(d)

Figure 4.10 Response of a space-time modulated metasurface with space-time varying permittivity according to (3.1), showing (a) the scattered fields at two time instants. (b) Transmitted and reflected fields at $z= \pm z_{0}$ for individual harmonics, (c) the spatial Fourier transform of the transmitted and reflected fields at $z=0_{+}$and $z=0_{-}$, respectively. (d) The refraction angles of each each harmonic components in reflection and transmission. The pumping frequency $\omega_{m}=0.025 \omega_{0}=5.75 \mathrm{THz}$ and spatial modulation $\beta_{m}=10 \pi / \ell . \mathcal{F}_{t}\{\cdot\}$ and $\mathcal{F}_{x}\{\cdot\}$ denote temporal and spatial Fourier transforms, respectively. Figure sourced from [2]. 
an almost complete down or up-conversion, consistent with the time-only modulated case of Fig. 4.9. This suggests that the spatial frequency of the perturbation, $\beta_{m}$, in $\epsilon_{r}(x, t)$ has little to no effect on the strength of the newly generated harmonics, and it only controls their respective refraction angles following (3.23). This phenomenon can also be seen with the reflected fields, where the distribution of the field between each frequency is very similar to the time modulated example.

A spatial Fourier transform could be performed on the spatial profiles at $z= \pm z_{0}$, but due to how distorted the $n= \pm 2$ harmonics are at this point it is more beneficial to analyze the fields closer to the metasurface. Fig. 4.10(c) shows the spatial Fourier transforms of the transmitted and reflected fields just after and before the metasurface at $z=0_{+}$and $z=0_{-}$, respectively, for the first few dominant harmonics. The locations $k_{x 0, n}$ of the peaks of the $n^{\text {th }}$ harmonics are then used to compute the refraction angles using Eq. (3.26) which are shown in Fig. 4.10(d) for both reflection and transmission fields. An excellent agreement is observed with the theoretical predictions of (3.23). Recall that this analysis is only valid for small diverging angles and must be verified to accurately determine the proper refraction angles.

absorbed, due to large refraction angles. 


\section{Chapter 5 Conclusions and Future Work}

\subsection{Discussion}

An explicit FDTD model of a space-time modulated Huygens' metasurface has been proposed and numerically demonstrated using the GSTC equations. The surface susceptibilities of a typical all-dielectric Huygens' unit cell at optical frequencies were extracted for different material permittivities. This permittivity was modulated in both space and time to emulate a travelling-wave type spatio-temporal perturbation in the metasurface. By mapping the material permittivity to the parameters of both the electric and magnetic Lorentzian surface susceptibilities the problem was formulated into a set of second-order equations with nonconstant coefficients. This set of equations were solved using an explicit finite-difference technique for a variety of incident fields and modulation schemes.

The model was initially tested using a single-point simulation, where only one point along the metasurface is solved for a normally incident plane wave. The first simulation was of a static (non-modulated) metasurface with an incident monochromatic field that slowly increased from zero to its full value. The model produced both a transmitted forwardpropagating wave and a reflected reverse-propagating wave that reached a steady-state value after some distortion caused by the Lorentzian susceptibilities. The same incident monochromatic field was then applied to a time-modulated metasurface to provide a comparison between the different output waveforms at steady-state. The simulation showed that both the transmitted and reflected fields produced harmonics at the expected frequencies, which were observed using a temporal FFT.

The metasurface model was then analyzed using two different 2D simulation schemes. The first method that was used was to propagate the time-domain output fields from a normally incident Gaussian beam in the frequency domain. The output fields of both a static metasurface and a space-time modulated metasurface were converted to the frequency domain and each of the harmonics were propagated independently in separate regions. It was found that the output of the static metasurface propagated normal to the surface with a peak power that was the same as the single-point simulation. The harmonics from the space-time modulated metasurface, however, all propagated with different peak powers and different angles of refraction, which matched the Floquet mode's expected outgoing angles. This showed that the harmnonics produced from the metasurface are angularly separated in

space when both a spatial and temporal modulation is applied to the metasurface. This also proved that the technique to propagate individual harmonic components from a broadband 
time-domain field works very well and is a fast and simple alternative to the Yee cell for steady-state solutions.

The second simulation scheme was using a 2D Yee cell to propagate the transmitted and reflected fields in the time domain. A static metasurface was tested using a normally incident plane wave and a Gaussian beam, and the spatial Fourier transforms of the outputs at a set distance away from the surface were compared with the theoretical profiles in the spectral domain to prove that the propagation was accurate. Another test of the accuracy of the propagation was conducted by applying a pulsed Gaussian beam to the static metasurface for different Yee cell mesh densities. It was found that the accuracy of the results converged to a set mesh density, which was chosen for all simulations to provide both accurate and fast results.

Once the accuracy of the Yee cell was verified, a normally incident pulsed Gaussian beam was applied to a time-modulated metasurface. The resulting field generated several harmonics in the pulsed Gaussian, which the temporal Fourier transform illustrated. This example proved that the model is able to handle broadband input sources even when a temporal modulation is applied to the metasurface.

The model was then tested using a normally incident Gaussian beam on a space-time modulated metasurface. From the simulation the fringing effects of the output waveform were observed and the spatial frequency profiles were extracted. From these profiles, the angles of refraction of the harmonics were extracted, which matched the theoretical values.

This model has been proved to be very versatile in capturing the effects of a space-time modulated metasurface with a normally incident arbitrary time-domain field. In conjunction with the different analytical tools that were demonstrated in this thesis, this method represents a powerful and efficient tool to model and design modulated metasurfaces for electromagnetic wave control.

\subsection{Limitations}

There are a few limitations of the current metasurface model. One of the assumptions is that all output waves are locally normally outgoing plane-waves. This assumption is reasonable for uniform static metasurfaces and time-only modulated metasurfaces only because all of the harmonics are propagating normal to the surface. However, there may be some inaccuracies that are added when a space modulated or space-time modulated metasurface is analyzed because the harmonics have a non-zero angle of refraction. Similarly, it is assumed that the incident wave is normal to the plane of the metasurface. This limitation of the model means 
that it is not possible to analyze oblique-incident waves with the current model with a high degree of accuracy.

The other limitation of the model is that it was simplified to assume that the metasurface has an isotropic response. In order to analyze a metasurface that has a more general response, the surface susceptibilities have to be represented as full tensors and the bi-anisotropic terms, $\chi_{\mathrm{em}}$ and $\chi_{\mathrm{me}}$, have to be taken into account. This extension is straight forward.

\subsection{Future Work}

In order to capture the effects of a metasurface under a broader range of uses, the model needs to be expanded in a few different ways. One of the flaws of the model is that it is only able to handle fields with a normal incidence, therefore it is not able to accurately model the effects associated with fields that have large oblique incidences. Although this may require a rewrite of the formulation, it will provide more insight into non-reciprocal behaviour, especially when space-time modulation is taken into account. This non-reciprocal behaviour has been presented theoretically using Floquet analysis [27], but has not been verified using a simulation model.

Another reason why capturing oblique incidence is important is if we want to model multiple metasurfaces in the same simulation region in two or three dimensions, because an incident field can no longer be assumed completely known. When a wave travels through space, the wave's diffraction causes the wavefront to have different angles of propagation depending on where you are measuring. This can cause problems when analyzing the surface because an incident wave could have an angle of incidence that varies depending on where it interacts with the surface. Therefore proper care will need to be taken in order to properly solve this generally.

The effects of multiple metasurfaces with a set distance between them is also an area

of heavy interest with a lot of different setups that can be explored. As a simple example, a Fabry-Perot resonator could be constructed using two metasurfaces and the transmission as a function of the distance between the two surfaces could be analyzed. Further applications could easily be explored that cannot be easily done with a frequency-domain model, such as if the two metasurfaces both have a space-time modulation. Such a device may be useful for frequency selection or frequency conversion.

Another area of research that may prove useful is to capture the effects of a non-linear metasurface in the model. A non-linear metasurface model has already been discussed in a recent work [28], however it does not take into account fields with an oblique angle of 
incidence and does not account for a more practical third order nonlinearity. One area of study that may prove interesting is the effects of a non-linear metasurface with a space-time modulation induced, where both the effects of a non-linear surface and of a time-modulated surface can only be simulated in the time domain.

One more assumption that needs to be broadened in order to handle a more general metasurface is to take into account the bi-isotropic surface susceptibilities $\chi_{\mathrm{em}}$ and $\chi_{\mathrm{me}}$. Although this will increase the complexity of the model, it will also provide a more accurate picture of the effects of wide varieties of EM metasurfaces.

One direction of research is to design and test a practical implementation of a space-time varying metasurface. If a silicon based metasurface is implemented, the carrier densities can be varied using external voltage biases to emulate a space-time perturbation of the surface susceptibilities. If the effect of this is too weak, a stronger pumping signal in a nonlinear material can cause a modulation of the constitutive parameters of the material. These small signals may propagate and interact with the incident fields, resulting in harmonic frequency generation. The research would touch upon many different areas, such as the design and fabrication of a practical metasurface, the extraction of the surface susceptibilities, as well as comparing the results of the physical metasurface under excitation with the results from the model. 


\section{Bibliography}

[1] T. Smy and S. Gupta, "Exact finite-difference time-domain modelling of broadband huygens' metasurfaces with lorentz dispersions," ArXiv:1609.05575, Sept. 2016.

[2] S. Stewart, S. Gupta, and T. Smy, "Finite-difference time-domain (fdtd) modelling of space-time modulated metasurfaces," arXiv:1612.0208\%, Dec. 2016.

[3] S. Stewart, T. Smy, and S. Gupta, "Semi-analytical finite-difference technique for steadystate field characterization of space-time modulated huygens' metasurfaces," in 2017 IEEE AP-S Symposium on Antennas and Propagation and USNC-URSI Radio Science Meeting, Jul 2017.

[4] C. Holloway, E. F. Kuester, J. Gordon, J. O'Hara, J. Booth, and D. Smith, "An overview of the theory and applications of metasurfaces: The two-dimensional equivalents of metamaterials," IEEE Antennas Propag. Mag., vol. 54, no. 2, pp. 10-35, April 2012.

[5] N. Yu and F. Capasso, "Flat optics with designer metasurfaces," Nature Materials, vol. 13, April 2014.

[6] K. Achouri, M. Salem, and C. Caloz, "General metasurface synthesis based on susceptibility tensors," IEEE Trans. Antennas Propag., vol. 63, no. 7, pp. 2977-2991, July 2015.

[7] V. S. Asadchy, I. A. Faniayeu, Y. Ra'di, S. A. Khakhomov, I. V. Semchenko, and S. A. Tretyakov, "Broadband reflectionless metasheets: Frequency-selective transmission and perfect absorption," Phys. Rev. X, vol. 5, p. 031005, Jul 2015.

[8] C. Pfeiffer and A. Grbic, "Metamaterial huygens' surfaces: Tailoring wave fronts with reflectionless sheets," Phys. Rev. Lett., vol. 110, p. 197401, May 2013.

[9] C. Holloway, E. F. Kuester, J. Gordon, J. O'Hara, J. Booth, and D. Smith, "An overview of the theory and applications of metasurfaces: The two-dimensional equivalents of metamaterials," Antennas and Propagation Magazine, IEEE, vol. 54, no. 2, pp. 10-35, April 2012.

[10] S. A. Tretyakov, "Metasurfaces for general transformations of electromagnetic fields," Philosophical Transactions of the Royal Society of London A: Mathematical, Physical and Engineering Sciences, vol. 373, no. 2049, 2015. 
[11] Y. Yang, H. Wang, Z. X. F. Yu, and H. Chen, "A metasurface carpet cloak for electromagnetic, acoustic and water waves," Scientific Reports., no. 6, pp. 1-6, Jan. 2016.

[12] G. Zheng, H. Muhlenbernd, M. Kenney, G. Li, T. Zentgraf, and S. Zhang, "Metasurface holograms reaching 80\% efficiency," Nat. Nanotech., no. 43, pp. 308-312, Feb. 2015.

[13] M. Kerker, The Scattering of Light and Other Electromagnetic Radiation. Academic Press, New York, 1969.

[14] M. Decker, I. Staude, M. Falkner, J. Dominguez, D. N. Neshev, I. Brener, T. Pertsch, and Y. S. Kivshar, "High-efficiency dielectric huygens' surfaces," Adv. Opt. Mater., vol. 3, no. 6, pp. 813-820, 2015.

[15] A. Arbabi, Y. Horie, M. Bagheri, and A. Faraon, "Complete control of polarization and phase of light with high efficiency and sub-wavelength spatial resolution," arXiv:1411.1494, pp. 4308-4315, Nov 2014.

[16] N. Yu, P. Genevet, M. A. Kats, F. Aieta, J.-P. Tetienne, F. Capasso, and Z. Gaburro, "Light propagation with phase discontinuities: Generalized laws of reflection and refraction," Science, vol. 334, no. 6054, pp. 333-337, 2011.

[17] Y. Hadad, D. L. Sounas, and A. Alu, "Space-time gradient metasurfaces," Phys. Rev. $B$, vol. 92, p. 100304, Sep 2015.

[18] A. Shaltout, A. Kildishev, and V. Shalaev, "Time-varying metasurfaces and lorentz non-reciprocity," Opt. Mater. Express, vol. 5, no. 11, pp. 2459-2467, Nov 2015.

[19] M. M. Idemen, Discontinuities in the Electromagnetic Field. John Wiley \& Sons, 2011.

[20] S. Gupta, "Generalized spatial talbot effect based on all-dielectric metasurfaces," arXiv:1605.03605, May 2016.

[21] Y. Vahabzadeh, N. Chamanara, and C. Caloz, "Space-time varying metasurface simulation in finite difference time domain," ArXiv:1701.08\%60, Jan. 2017.

[22] E. F. Kuester, M. A. Mohamed, M. Piket-May, and C. L. Holloway, "Averaged transition conditions for electromagnetic fields at a metafilm," IEEE Transactions on Antennas and Propagation, vol. 51, no. 10, pp. 2641-2651, Oct 2003.

[23] E. Rothwell and M. Cloud, Electromagnetics. CRC Press, 2001.

[24] J. Goodman, Introduction to Fourier Optics. Roberts and Company Publishers; 3rd Edition edition, 2004. 
[25] B. E. A. Saleh and M. C. Teich, Fundamentals of Photonics, 2nd ed. Wiley-Interscience, 2007.

[26] A. Taflove and S. Hagness, "Computational electrodynamics: The finite-difference timedomain method," 2000.

[27] S. Gupta, S. Stewart, and T. Smy, "Space-time modulation induced non-reciprocity in electromagnetic metasurfaces," arXiv:1703.10823, Mar. 2017.

[28] K. Achouri, Y. Vahabzabeg, and C. Caloz, "Mathematical synthesis and analysis of nonlinear metasurfaces," arXiv:1703.09082, Mar. 2017. 


\section{Appendix A Extension to Dual-Lorentz Response}

Consider an electric surface susceptibility consisting of two Lorentzian contributions such that $\tilde{\chi}_{\mathrm{ee}}=\tilde{\chi}_{\mathrm{ee}, 1}+\tilde{\chi}_{\mathrm{ee}, 2}$, following (3.3). Its relates the total polarization density on the metasurface with the exciting E-fields, as $\tilde{P}_{0}(\omega)=\epsilon_{0} \tilde{\chi}_{\mathrm{ee}} \tilde{E}_{0}(\omega)$. In this case, the total polarization can be broken into two parts as $\tilde{P}_{0}(\omega)=\tilde{P}_{01}(\omega)+\tilde{P}_{02}(\omega)$, where $\tilde{P}_{01}(\omega)=\tilde{\chi}_{\text {ee, } 1} \tilde{E}_{0}(\omega)$ and $\tilde{P}_{02}(\omega)=\tilde{\chi}_{\text {ee, } 2} \tilde{E}_{0}(\omega)$, respectively. Similar decomposition can be done for the magnetic surface susceptibilities following an identical procedure. Now each electric and magnetic polarization term, can be written in the time domain using the Lorentzian form, for incident, transmitted and reflected fields, given in matrix form as
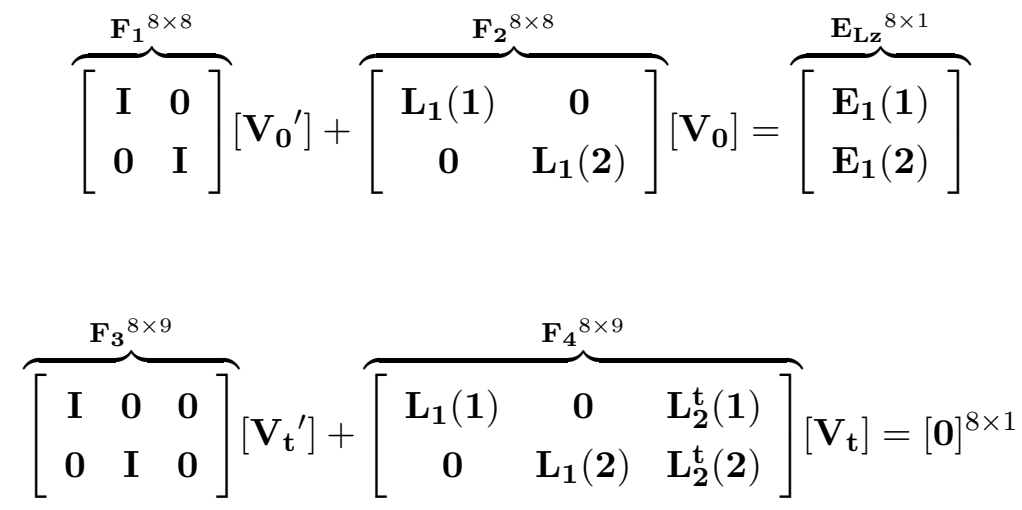

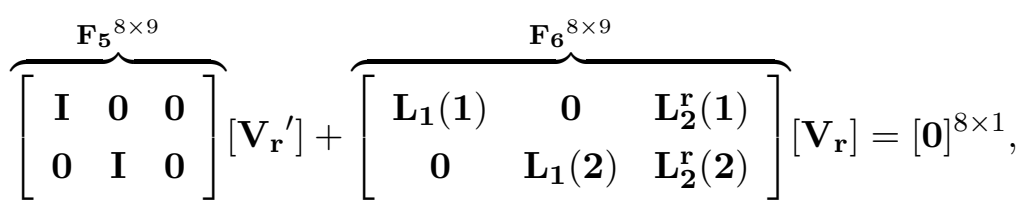

where the above matrices are given by

$$
\mathbf{L}_{\mathbf{1}}(i)=\left[\begin{array}{cccc}
\alpha_{e i} & -\omega_{e 0, i} & 0 & 0 \\
\omega_{e 0, i} & \frac{\omega_{e 0, i}^{\prime}}{\omega_{e 0, i}} & 0 & 0 \\
0 & 0 & \alpha_{m i} & -\omega_{m 0, i} \\
0 & 0 & \omega_{m 0, i} & \frac{\omega_{m 0, i}^{\prime}}{\omega_{m 0, i}}
\end{array}\right]
$$




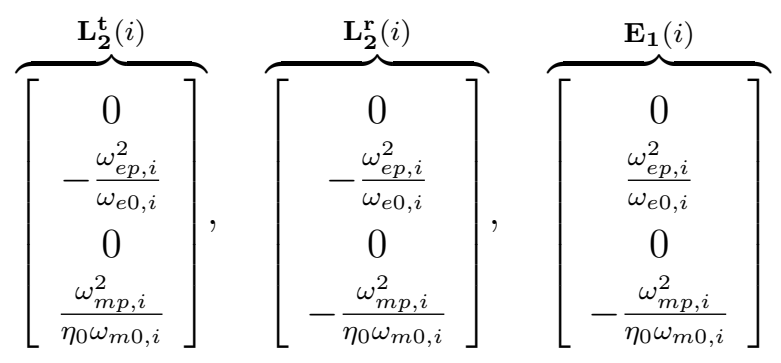

and

$$
\mathbf{V}_{\mathbf{0}}=\left[\begin{array}{c}
Q_{01} \\
\bar{Q}_{01} \\
M_{01} \\
\bar{M}_{01} \\
Q_{02} \\
\bar{Q}_{02} \\
M_{02} \\
\bar{M}_{02}
\end{array}\right] \mathbf{V}_{\mathbf{t}}=\left[\begin{array}{c}
Q_{t 1} \\
\bar{Q}_{t 1} \\
M_{t 1} \\
\bar{M}_{t 1} \\
Q_{t 2} \\
\bar{Q}_{t 2} \\
M_{t 2} \\
\bar{M}_{t 2} \\
E_{t}
\end{array}\right] \mathbf{V}_{\mathbf{r}}=\left[\begin{array}{c}
Q_{r 1} \\
\bar{Q}_{r 1} \\
M_{r 1} \\
\bar{M}_{r 1} \\
Q_{r 2} \\
\bar{Q}_{r 2} \\
M_{r 2} \\
\bar{M}_{r 2} \\
E_{r}
\end{array}\right]
$$

Finally, the two GSTC equations can also be written in terms of decomposed surface susceptibilities, as

$$
\begin{aligned}
&\left(\omega_{m 0,1} \bar{M}_{01}\right.\left.-\alpha_{m 1} M_{01}\right)+\left(\omega_{m 0,1} \bar{M}_{r 1}-\alpha_{m 1} M_{r 1}\right) \\
&+\left(\omega_{m 0,1} \bar{M}_{t 1}-\alpha_{m 1} M_{t 1}\right) \\
&\left(\omega_{m 0,2} \bar{M}_{02}-\alpha_{m, 2} M_{02}\right)+\left(\omega_{m 0,2} \bar{M}_{r 2}-\alpha_{m, 2} M_{r 2}\right) \\
&+\left(\omega_{m 0,2} \bar{M}_{t 2}-\alpha_{m, 2} M_{t 2}\right)=\frac{2}{\mu_{0}}\left(E_{t}-E_{r}-E_{0}\right), \\
&\left(\omega_{e 0,1} \bar{Q}_{01}-\alpha_{e 1} Q_{01}\right)+\left(\omega_{e 0,1} \bar{Q}_{r 1}-\alpha_{e 1} Q_{r 1}\right) \\
&+\left(\omega_{e 0,1} \bar{Q}_{t 1}-\alpha_{e 1} Q_{t 1}\right) \\
&\left(\omega_{e 0,2} \bar{Q}_{02}-\alpha_{e, 2} Q_{02}\right)+\left(\omega_{e 0,2} \bar{Q}_{r 2}-\alpha_{e, 2} Q_{r 2}\right) \\
&+\left(\omega_{e 0,2} \bar{Q}_{t 2}-\alpha_{e, 2} Q_{t 2}\right)=\frac{2}{\epsilon_{0} \eta_{0}}\left(E_{0}-E_{t}-E_{r}\right),
\end{aligned}
$$


Combining the Lorentzian relations with the GSTC equations, lead to a matrix equation given by

$$
[\mathbf{C}]\left[\begin{array}{c}
\mathbf{V}_{\mathbf{0}}^{\prime} \\
\mathbf{V}_{\mathbf{t}}^{\prime} \\
\mathbf{V}_{\mathbf{r}}^{\prime}
\end{array}\right]+[\mathbf{G}]\left[\begin{array}{c}
\mathbf{V}_{\mathbf{0}} \\
\mathbf{V}_{\mathbf{t}} \\
\mathbf{V}_{\mathbf{r}}
\end{array}\right]=\left[\begin{array}{c}
\mathbf{E}_{\mathbf{G}} \\
\mathbf{E}_{\mathbf{L z}} \\
\mathbf{0} \\
\mathbf{0}
\end{array}\right]
$$

where

$$
\begin{aligned}
& {[\mathbf{C}]=\left[\begin{array}{ccc}
\mathbf{0} & \mathbf{0} & \mathbf{0} \\
\mathbf{0} & \mathbf{0} & \mathbf{0} \\
\mathbf{F}_{1} & \mathbf{0} & \mathbf{0} \\
\mathbf{0} & \mathbf{F}_{3} & \mathbf{0} \\
\mathbf{0} & \mathbf{0} & \mathbf{F}_{5}
\end{array}\right],[\mathbf{G}]=\left[\begin{array}{ccc}
\mathbf{J}_{1} & \mathbf{J}_{2} & \mathbf{J}_{3} \\
\mathbf{K}_{1} & \mathbf{K}_{2} & \mathbf{K}_{3} \\
\mathbf{F}_{2} & \mathbf{0} & \mathbf{0} \\
\mathbf{0} & \mathbf{F}_{4} & \mathbf{0} \\
\mathbf{0} & \mathbf{0} & \mathbf{F}_{6}
\end{array}\right]} \\
& \mathbf{J}_{\mathbf{1}}=\left[\begin{array}{ll}
\mathbf{A}_{\mathbf{1}}(1) & \mathbf{A}_{\mathbf{1}}(2)
\end{array}\right], \mathbf{J}_{\mathbf{2}}=\left[\begin{array}{lll}
\mathbf{A}_{\mathbf{1}}(1) & \mathbf{A}_{\mathbf{1}}(2) & -2 / \mu_{0}
\end{array}\right], \\
& \mathbf{J}_{\mathbf{3}}=\left[\begin{array}{lll}
\mathbf{A}_{\mathbf{1}}(1) & \mathbf{A}_{\mathbf{1}}(2) & 2 / \mu_{0}
\end{array}\right], \mathbf{A}_{\mathbf{1}}(i)=\left[0,0,-\alpha_{m, i}, \omega_{m, i}\right]
\end{aligned}
$$

and,

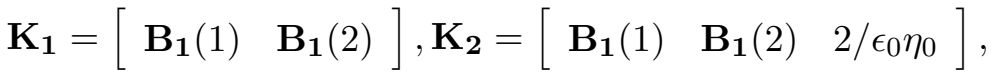

$$
\begin{aligned}
& \mathbf{K}_{\mathbf{3}}=\mathbf{K}_{\mathbf{2}}, \mathbf{B}_{\mathbf{1}}(i)=\left[-\alpha_{e, i}, \omega_{e, i}, 0,0\right], \mathbf{E}_{\mathbf{G}}\left[\begin{array}{c}
-\left(2 / \mu_{0}\right) E_{0} \\
\left(2 / \epsilon_{0} \eta_{0}\right) E_{0}
\end{array}\right] \text {. }
\end{aligned}
$$

Equation A.8 can now be solved using standard numerical techniques. While this derivation is shown here for two Lorentzian contributions in the susceptibilities for illustration, it can easily be extended to arbitrary $N$ terms. 